Copyright

by

Jackson A. Lewis

2000 


\title{
Finite Element Modeling and Active Control of an Inflated Torus Using Piezoelectric Devices
}

\author{
by \\ Jackson A. Lewis, B.S. \\ Thesis submitted to the Faculty of the \\ Virginia Polytechnic Institute and State University \\ in partial fulfillment of the requirements for the degree of \\ Master of Science \\ in
}

\section{Mechanical Engineering}

\author{
Daniel J. Inman, Chair \\ Donald J. Leo \\ Robert L. West
}

December 2000

Blacksburg, Virginia 


\section{Finite Element Modeling and Active Control of an Inflated Torus Using Piezoelectric Devices}

Approved by Advising Committee: 


\title{
Finite Element Modeling and Active Control of an Inflated Torus Using Piezoelectric Devices
}

\author{
Jackson A. Lewis, M.S. \\ Virginia Polytechnic Institute and State University, 2000 \\ Advisor: Daniel J. Inman
}

\begin{abstract}
Satellite antenna design requirements are driving the satellite size to proportions that cannot be launched into space using current technology. In order to reduce the launch size and mass of satellites, inflatable structures, also known as gossamer structures, are being considered. Inflatable space-based structures are susceptible to vibration disturbance due to their low stiffness and damping. This thesis discusses the structural dynamics and vibration suppression via piezoelectric actuators, using active control of an inflatable torus.
\end{abstract}

A commercial finite element package, ANSYS, is used to model the inflated torus. The effect of torus aspect ratio and inflation pressure on the vibratory response of the structure is investigated. The interaction with the torus of the surface-mounted piezoelectric patches, made of PVDF, is modeled using Euler-Bernoulli beam theory. A state space representation is created of the model in modal space and modal truncation is performed. Traditional control tools are used to suppress vibration in the structure. First observer-based full state feedback is used, then direct output velocity feedback is explored.

The aspect ratio of the torus is found to significantly influence the mode shapes. Toroids of small aspect ratios, skinny toroids, act like rings, but the mode shapes of toroids with large aspect ratios are much more complicated. For toroids of small 
aspect ratios, increasing the inflation pressure simply results in stiffening the ring, thereby increasing the natural frequencies. Increasing the pressure in toroids with large aspect ratios changes both the mode shapes and natural frequencies. The passive effect of PVDF on the dynamics of the torus is small, the mode shapes do not change and the frequencies are only slightly reduced. Active control of toroids with small aspect ratios using piezoelectric devices is effective. It may be more difficult to control toroids with large aspect ratios because the mode shapes are much more complicated than the simple ring modes found in toroids with small aspect ratios. 


\section{Acknowledgments}

First and foremost I would like to thank my parents for imbuing in me the value of education. I would like to thank my advisor, Dr. Daniel J. Inman, for his guidance throughout my work at the CIMSS. His support and understanding of my goals has been essential to this work. I would also like to thank my committee members Donald J. Leo and Robert L. West for their support and knowledgeable insights. Additional thanks go to Dr. Eric M. Austin who deserves much of the credit for starting me down the road toward graduate studies and this particular research project.

I would also like to extend my thanks to all my colleagues at CIMSS for their support. Their willingness to help with all manner of topics has been greatly appreciated. In particular I would like to thank Robert Brett Williams for all his help, without which I could never have made the progress I did.

Finally I would like to thank my loving wife, Erin McParland Lewis. Her understanding and compassion have truly enabled me to complete this thesis.

The work contained herein was supported by the Air Force Office of Scientific Research (AFOSR) under grant number F49620-99-1-0231; and by the Mechanical Engineering Department of Virginia Tech.

JACKSON A. LEWIS

Virginia Polytechnic Institute and State University

December 2000 


\section{Contents}

Abstract iv

Acknowledgments vi

List of Tables $\quad x$

List of Figures $\quad$ xi

$\begin{array}{lll}\text { Chapter } 1 & \text { Introduction } & 1\end{array}$

1.1 Motivation . . . . . . . . . . . . . . . . . . . 1

1.2 Overview of Inflatable Satellites $\ldots \ldots \ldots \ldots$

1.2 .1 Material . . . . . . . . . . . . . . . . 3

1.2.2 Pressurization and Deployment . . . . . . . . . . 4

1.2 .3 Ridigizable Structures $\ldots \ldots \ldots \ldots \ldots$

1.3 Literary Review . . . . . . . . . . . . . . . . . . . . . 5

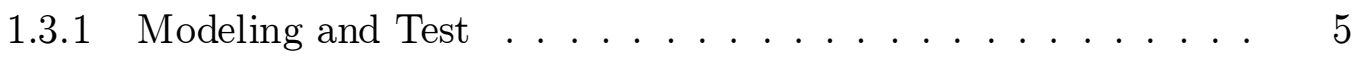

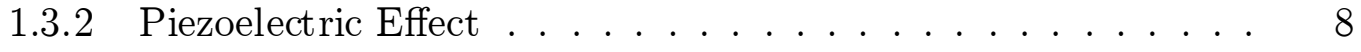

1.3 .3 Control Design . . . . . . . . . . . . . . . . . . 11

1.4 Overview of Thesis $\ldots \ldots \ldots \ldots \ldots$

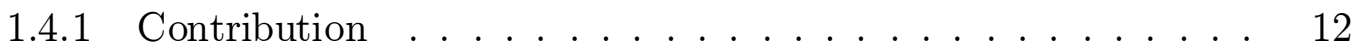

$1.4 .2 \quad$ Approach . . . . . . . . . . . . . . . . . 12

$\begin{array}{lll}\text { Chapter } 2 & \text { Modeling of a Torus } & 14\end{array}$

2.1 Introduction . . . . . . . . . . . . . . . . . . 14 
2.2 Finite Element Modeling . . . . . . . . . . . . . . . . . . 15

2.2 .1 Mass and Stiffness Extraction . . . . . . . . . . . . 17

2.3 Finite Element Model Verification . . . . . . . . . . . . . . . 18

2.3 .1 Tensioned String $\ldots \ldots \ldots$

2.3.2 Tensioned Membrane . . . . . . . . . . . . . . . . . 20

2.3 .3 Model Convergence . . . . . . . . . . . . . . . . . . . . 24

2.3 .4 External Verification . . . . . . . . . . . . . . . 24

2.4 Problem Formulation . . . . . . . . . . . . . . . . . . . 26

2.4 .1 Mass Condensation . . . . . . . . . . . . . . . . . . 26

2.4 .2 Eigenvalue Solution . . . . . . . . . . . . . . . . 27

2.4 .3 Modal Equations . . . . . . . . . . . . . . . . . . . . . 29

2.4.4 State Space Formulation . . . . . . . . . . . . . . . . 30

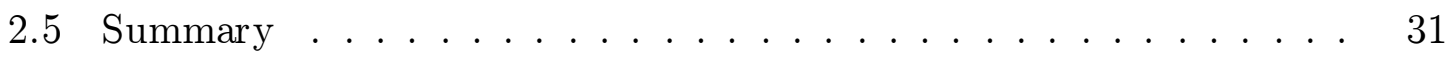

Chapter 3 Actuators and Control Design 32

3.1 Introduction . . . . . . . . . . . . . . . . . . 32

3.2 Piezoelectric Modeling . . . . . . . . . . . . . . . . . 32

3.3 Control Design . . . . . . . . . . . . . . . . . . . 34

3.3 .1 Actuator Placement . . . . . . . . . . . . . . 34

3.3.2 Full State Feedback Modal Controller . . . . . . . . . . . . 37

3.3.3 Observer-Based Feedback Design _. . . . . . . . . . . . 39

3.3.4 Observation Spillover . . . . . . . . . . . . . . . . . . . 42

3.3.5 Optimal Control . . . . . . . . . . . . . . . . . . 44 43

3.3 .6 Velocity Feedback . . . . . . . . . . . . . . . . . . 43

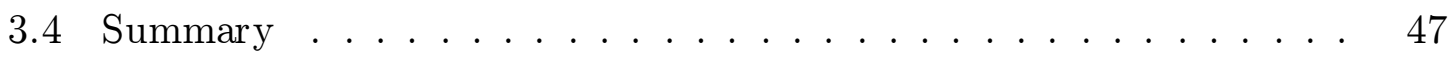

$\begin{array}{lll}\text { Chapter } 4 \text { Results } & 48\end{array}$

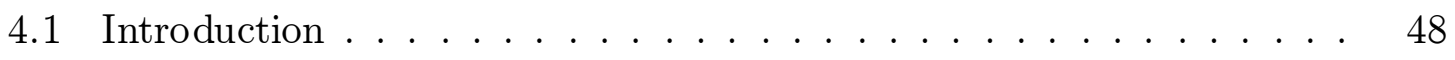

4.2 Effect of Torus Aspect Ratio on Mode Shapes . . . . . . . . . . 48

4.3 Dynamic Effect of Passive PVDF . . . . . . . . . . . . 50

4.4 Effect of Pressure on Natural Frequencies . . . . . . . . . . . 51 
4.5 Open Loop Response of Torus . . . . . . . . . . . . . . . . . 54

4.6 Observer Based State Feedback Control . . . . . . . . . . . . . . 54

4.6.1 Effect of Observer Spillover _. . . . . . . . . . . . 61

4.7 Results of Velocity Feedback Control . . . . . . . . . . . . 63

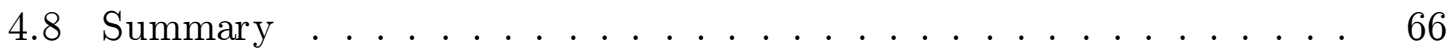

$\begin{array}{lll}\text { Chapter } 5 & \text { Conclusions } & 67\end{array}$

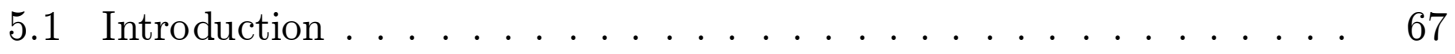

5.2 Key Results . . . . . . . . . . . . . . . . . . . . 67

5.3 Future Work . . . . . . . . . . . . . . . . . . . . . . . 68

$\begin{array}{ll}\text { Bibliography } & \mathbf{7 0}\end{array}$

$\begin{array}{ll}\text { Appendix A Mode Shapes } & 73\end{array}$

$\begin{array}{lll}\text { Appendix B } & 88 \text { MATLAB Mesh Generation }\end{array}$

$\begin{array}{lll}\text { Appendix C MATLAB Eigenvalue Solution } & 92\end{array}$

Appendix D MATLAB Mode Shape Post Processor 96

Appendix E MATLAB Settle Time Calculation 100

Appendix F MATLAB Observer Feedback Simulation 101

$\begin{array}{ll}\text { Appendix G MATLAB Velocity Feedback Simulation } & 107\end{array}$

$\begin{array}{ll}\text { Vita } & 112\end{array}$ 


\section{List of Tables}

2.1 Finite Element Model Material Properties . . . . . . . . . . 15

2.2 Finite Element Model Geometric Properties . . . . . . . . . . . . . . 15

2.3 Properties of String . . . . . . . . . . . . . . . . . . . . . . 19

2.4 First Three Modes of Vibration of a String in Tension . . . . . . . . . 20

2.5 Properties of Membrane . . . . . . . . . . . . . . . 21

2.6 Analytical and Finite Element Natural Frequencies of Tensioned Mem-

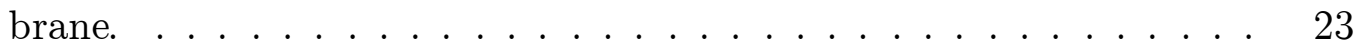

2.7 Torus Model Convergence at .5 psi. . . . . . . . . . . . . . . . . 24

2.8 Verification Model Properties . . . . . . . . . . . . . 25

2.9 Comparison of FEA and Liepins Model . . . . . . . . . . . . 25

3.1 Variable Definitions and Values for Bernoulli-Euler Beam Model . . . 34

4.1 Frequencies for Toroids of Different Aspect Ratios . . . . . . . . . . . 49

4.2 Controller Results . . . . . . . . . . . . . . . . . . . . 66 


\section{List of Figures}

1.1 Diagram of Inflatable Antenna used in IAE. . . . . . . . . . . . . 3

1.2 Definition of Tor us Dimensions. . . . . . . . . . . . . . . 6

1.3 Five Meter Concentrator and Torus at NASA. . . . . . . . . . . . . . 7

1.4 1-D Model of Force Output from a Piezoelectric Device. . . . . . . . . 10

2.1 Locations of PVDF on Torus. . . . . . . . . . . . . . 16

2.2 Membrane Coupon Under Non-Uniform Tension $\mathrm{P}_{1}$ and $\mathrm{P}_{2} \ldots \ldots$. . . 21

2.3 (a) Boundary Conditions for Static Analysis

(b) Boundary Conditions for Dynamic Analysis . . . . . . . . . . . . 22

3.1 Open Loop Impulse Response to In-Plane Excitation. . . . . . . . . . 37

4.1 Effect of Passive PVDF Patches on Frequency. . . . . . . . . . . . . 51

4.2 Effect of Pressure on Small Aspect Ratio (0.04) Torus Natural Fre-

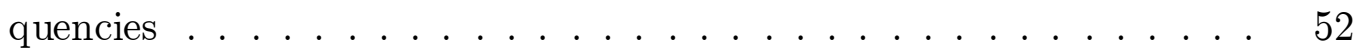

4.3 Effect of Pressure on Small Aspect Ratio (0.04) Tor us Mode Shapes . 52

4.4 Effect of Pressure on Large Aspect Ratio (0.32) Torus Natural Fre-

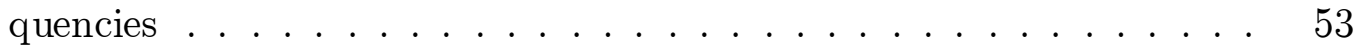

4.5 Effect of Pressure on Large Aspect Ratio (0.32) Torus Mode Shapes . 53

4.6 Open Loop Impulse Response to In-Plane Excitation. . . . . . . . . . 55

4.7 Open Loop Frequency Response to In-Plane Excitation. $\mathrm{Y}(1)=$ In-Plane-Response, $\mathrm{Y}(2)=$ Out-of-Plane Response $\ldots . . . . \quad 55$

4.8 Open Loop Impulse Response to Out-of-Plane Excitation. . . . . . . 56 
4.9 Open Loop Frequency Response to Out-of-Plane Excitation. $\mathrm{Y}(1)=$ Out-of-Plane Response, $\mathrm{Y}(2)=$ In-Plane Response . . . . . . . . 56

4.10 Observer Based Closed Loop Impulse Response to In-Plane Excitation, Observer Designed Using Pole Placement. . . . . . . . . . . . . . . 57

4.11 Observer Based Closed Loop Output Force for In-Plane Excitation, Observer Designed Using Pole Placement. . . . . . . . . . . . . . . . 57

4.12 Observer Based Closed Loop Impulse Response to Out-of-Plane Excitation, Observer Designed Using Pole Placement. . . . . . . . . . . . 58

4.13 Observer Based Closed Loop Output Force for Out-of-Plane Excitation, Observer Designed Using Pole Placement. . . . . . . . . . . . . 58

4.14 Observer Based Closed Loop Impulse Response to In-Plane Excitation, Observer Designed Using Optimal Control. . . . . . . . . . . . . . . 59

4.15 Observer Based Closed Loop Output Force for In-Plane Excitation, Observer Designed Using Optimal Control. . . . . . . . . . . . . . . . 59

4.16 Observer Based Closed Loop Impulse Response to Out-of-Plane Excitation, Observer Designed Using Optimal Control. . . . . . . . . . . 60

4.17 Observer Based Closed Loop Output Force for Out-of-Plane Excitation, Observer Designed Using Optimal Control. . . . . . . . . . . . . 60

4.18 Impulse Response Considering Residual Modes using Pole Placement Techniques. ........................ 62

4.19 Impulse Response Considering Residual Modes using Optimal Control. 62

4.20 Velocity Feedback Closed Loop Impulse Response to In-Plane Excitation. 64

4.21 Velocity Feedback Closed Loop Output Force for In-Plane Excitation. 64

4.22 Velocity Feedback Closed Loop Impulse Response to Out-of-Plane Ex-

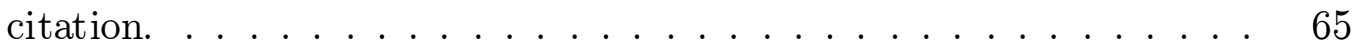

4.23 Velocity Feedback Closed Loop Output Force for Out-of-Plane Excita-

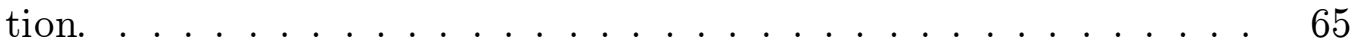




\section{Chapter 1}

\section{Introduction}

\subsection{Motivation}

Satellite antenna design requirements are driving the satellite size to proportions that cannot be launched into space using current technology. This problem has introduced a revolutionary change in current satellite design. Instead of using large rigid antennas, new satellites may be inflatable. Inflatable satellites, also known as gossamer structures, have many advantages, the most important being the potential for significantly lower cost, volume, and mass. Inflatable structures also have additional challenges. It is well known that many traditional space structures have encountered vibration problems. The Hubble Space telescope was susceptible to vibrations induced by thermal shock as it moved in and out of the earth's shadow, rendering it useless for fifteen minutes at a time [20]. Typically space structures have vibration problems due to low structural stiffness and the lack of air damping in the vacuum of space. Inflatable satellites are likely to be even more susceptible to vibration problems because of low structural stiffness and material damping. There are two main types of vibration excitation for satellites: shock and harmonic. Shock disturbances can come from a number of sources such as meteoroid impact, thermal shock, and satellite repositioning. Harmonic excitations typically come from rotating unbalance on the satellite itself, such as rotating imbalance in a reaction wheel. Attenuating these disturbances is paramount to maintaining their performance. Traditional pas- 
sive damping measures typically add mass and or stiffness to the structure, which is not desirable. Using active control and smart materials is an approach that has the potential to attenuate vibration without significantly increasing the mass or stiffness of the structure.

The United States Air Force has decided to investigate the use of active vibration control with smart materials for inflatable satellites. The Center for Intelligent Material Systems and Structures at Virginia Polytechnic Institute and State University has been chosen to work with the Air Force Institute of Technology to examine the feasibility of vibration control of inflatable satellites using piezoelectric devices.

The purpose of this thesis will be to create a model of an inflated torus with attached piezopolymer patches. The interaction of the piezopolymer patches with the host structure, the inflated torus, will be examined. Finally a simple control system will be implemented to demonstrate the feasibility of suppressing vibration in the torus.

\subsection{Overview of Inflatable Satellites}

Inflatable technology is not new. Inflatable structures were in orbit in 1960 with NASA's ECHO I and ECHO II project. The ECHO spacecraft were communications satellites. The Explorer IX and XIX inflatable spacecraft were used for high altitude atmospheric studies. These spacecraft ranged in size from 12 feet in diameter and 34 pounds for the Explorer spacecraft to 135 feet in diameter and 580 pounds for ECHO II. Lack of understanding of inflatables and the overestimation of the meteoroid threat led the space industry to abandon inflatables until recently [2].

The satellite that will be considered here is based on the Inflatable Antenna Experiment (IAE) shown in Figure 1.1 which was performed in 1996 [21]. L'Garde and NASA JPL worked together to perform the experiment. Antennas of this type could be used for space and mobile communications, earth observations, astronomical observations, and space-based radar.

The satellite is made up of four major components: lens, torus, struts, and 


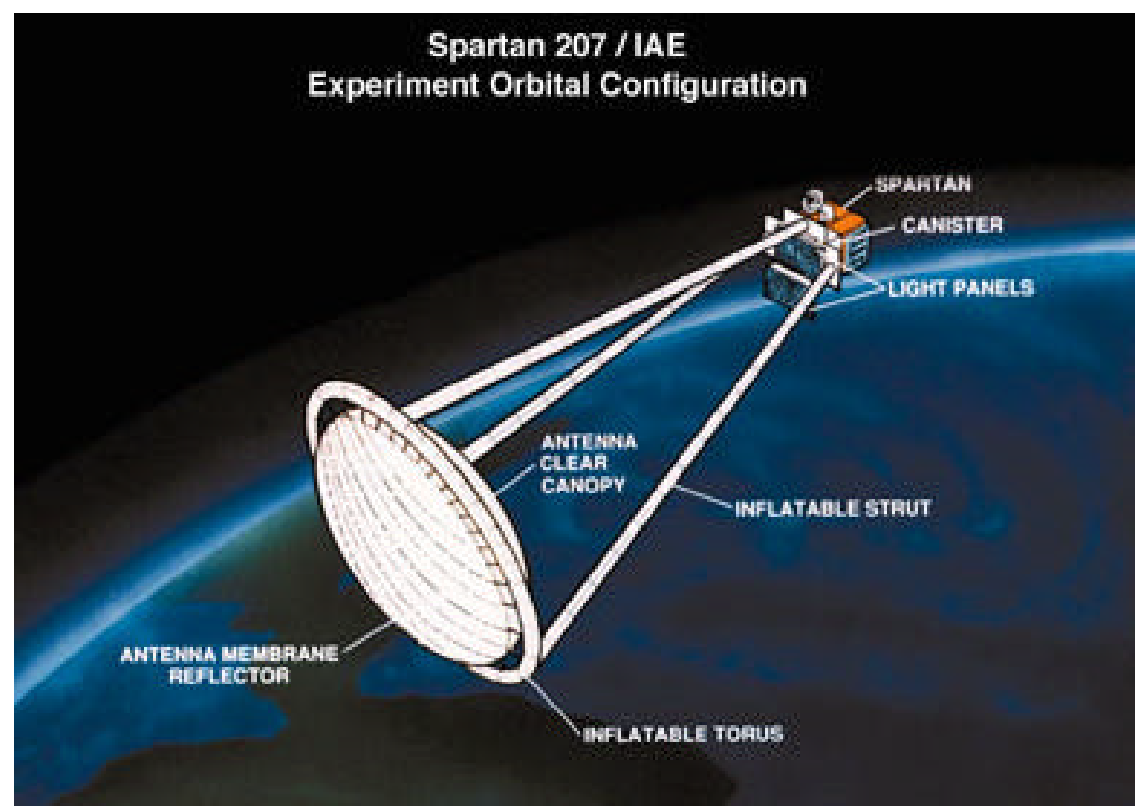

Figure 1.1: Diagram of Inflatable Antenna used in IAE.

body. The inflated parabolic lens is about 14 meters in diameter. The lens is constructed of a clear canopy on the side facing the body and a reflective surface on the other. The lens is supported with an inflated torus by means of 62 flexible adjustable ties. The torus has a major diameter of 15.2 meters and a cross-section diameter of 0.61 meters $[7,8]$. The three inflated struts are 28 meters long and attach to the torus by means of rigid adapter rings which are used to interface the strut-end fittings to fittings that were bonded on the torus [6]. The struts are attached to the body using additional fittings. The body contains the communications hardware, positioning hardware (reaction wheels), and inflation system. The packaged predeployment dimensions of the antenna are 6'x3'x3'. The total weight of the inflatable antenna structure is $60 \mathrm{~kg}[7]$.

\subsubsection{Material}

The IAE struts and torus are constructed with $0.3 \mathrm{~mm}$ (.012") thick neoprene rubber coated kevlar fabric. Neoprene coated kevlar is readily available, strong, leak-free, 
bondable, flexible, and reasonably priced [7]. The material was selected because

L'Garde, the antenna manufacturer, had extensive experience using the material for other projects.

The reflector is made of 6.35 micron (0.00025") Mylar film with vapor deposited aluminum to form the reflective surface. The reflector is fabricated by joining flat pieshaped gores, which are joined using Mylar tape. The canopy is made from the same Mylar film without the addition of the reflective coating. The reflector is stressed, via pressurization, to $6.89 \mathrm{MPa}$ because this stress level has been shown to remove the packaging wrinkles $[8]$.

The analysis used in this paper will be based on the material Kapton, a thin polyimide film made by DuPont. The thickness of Kapton for this analysis is $0.003 "$. Kapton is a material that shows significant future promise for application in inflatable space applications. It is stable at both high and low temperature extremes and is tough and abrasion resistant. The analy tical disadvantage of Kapton is that it exhibits significant non-linear properties, being both temperature and frequency dependent.

\subsubsection{Pressurization and Deployment}

The IAE satellite structure, torus and struts, are inflated with nitrogen to an operating pressure of about $2.9 \mathrm{psi}$. The lens is operated at a pressure of $0.29 \mathrm{e}-3 \mathrm{psi}$, much lower than the rest of the structure [7]. Using the material Kapton, for the structure but not the lens, the satellite structure would be pressurized to $0.5 \mathrm{psi}$, not to exceed 1 psi [23]. These pressurization levels, of about 0.5 psi, will be used in the following analysis. The inflation pressure is maintained by an on-board control system and bottle of pressurized nitrogen. As the satellite moves in and out of the sun, nitrogen may be vented and refilled to account for the change in pressure [23].

Deployment control is important for inflatable antennas to avoid damaging them. The deployment sequence planned for the IAE was not successful due to the presence of residual gas in the structure, which deployed it in an unanticipated manner. Fortunately the antenna did deploy successfully, but in an uncontrolled sequence. More development of ascent venting and deployment techniques is required 
for future missions $[6]$.

\subsubsection{Ridigizable Structures}

The satellites described here need to maintain their internal pressure to have structural stiffness. Although, as mentioned above, the risk of meteroids and other objects impacting the structures is relatively small, it may be desirable to have structures that are ridigizable. These structures would deploy like the inflatables described above, but would then ridigize so they no longer needed internal pressurization to maintain their shape. Different ridigization techniques are being examined, one of the most prevalent being curing the material with ultraviolet light. The UV radiation in space can be very intense and is an excellent source of energy for rigidizing a structure. Other possibilities are thermoset materials, which could require a large amount of thermal energy to cure, or water-based materials that harden as they dry [4].

\subsection{Literary Review}

\subsubsection{Modeling and Test}

A torus, although a fairly simple structure in concept, is actually numerically very challenging. Few fully analytical solutions for the vibrations of toroids exist due to the complexity of the problem. Numerical solutions for the axisymmetric vibrations of toroids were examined in the 1960's by A. A. Liepins and P. F. Jordan [16, 17, 18, 13, 14]. Liepins uses finite difference methods and Sanders linear shell theory to explore the free vibrations of the prestressed toroidal shell [17]. Liepins is most concerned with axisymmetric cross-sectional deformation of the torus, the deformation of the tube, but also discusses in limited detail the global deformation of the torus, the ring modes. The ring modes are approximated by the vibrations of a thin ring without prestress. The relative importance of the types of mode depend on the torus aspect ratio, defined here as the radius of the tube divided by the radius of the ring as shown in Figure 1.2. 


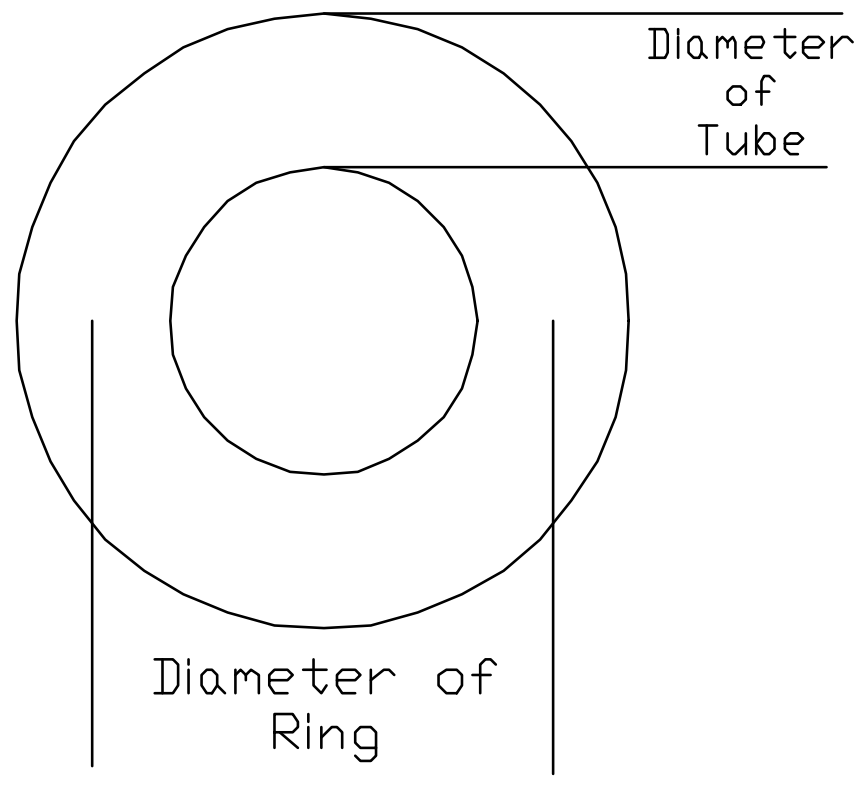

Figure 1.2: Definition of Torus Dimensions.

Liepins also created numerical models of toroidal shells with non-constant wall thicknesses and various types of support located at the inner and outer circumferences of the torus [18]. A test was also performed by Jordan on toroids similar to those modeled by Liepins [13]. Jordan found that the fundamental modes of the structure agreed well with predictions obtained from static asymptotic analysis using the Rayleigh quotient. Interestingly he found significant acoustic structural interactions. He proved the existence of the interactions by replacing part of the air in the test object with helium.

Williams analytically investigated the modes of a tensioned membrane [24]. He then examined the effect on the modes with a piezopolymer patch attached to the membrane. He found that adding the piezoelectric patch to the membrane reduced the frequency slightly but did not appreciably change the mode shapes. He also found that the distribution of mass on the patch was significant, for instance a thin large patch of the same mass as a thick small patch produced a different result. The mass that was more concentrated in the center of the patch, the thick small patch, lowered the frequency more than the more dispersed mass. His final conclusion was 


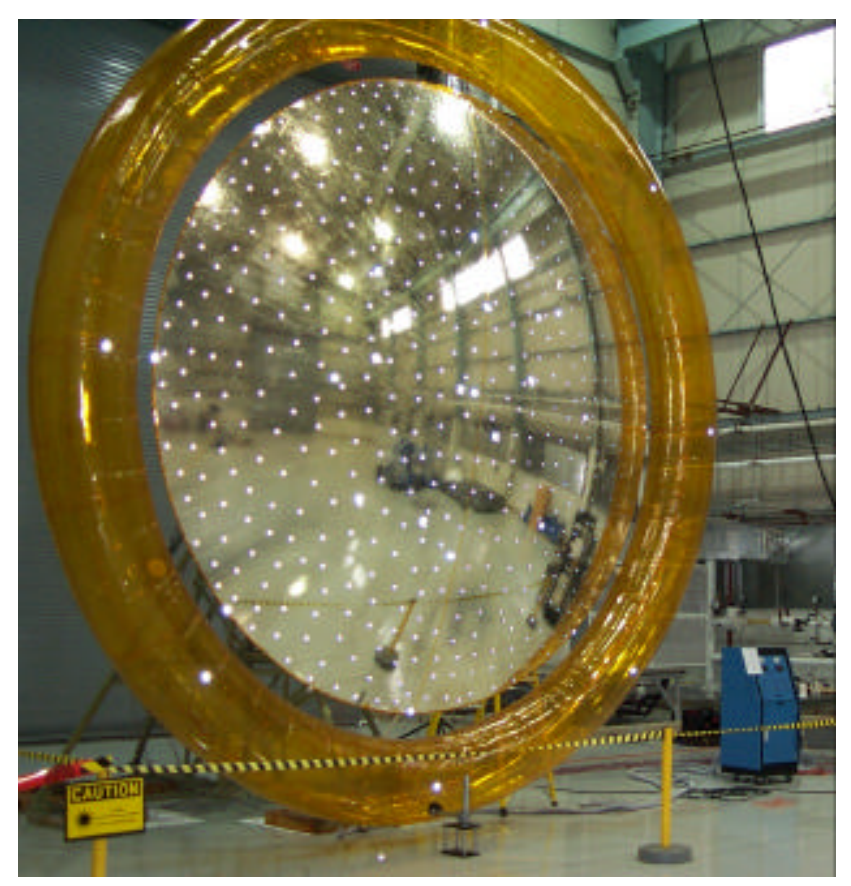

\section{Figure 1.3: Five Meter Concentrator and Torus at NASA.}

that membrane theory was not appropriate for modeling inflated structures with bonded piezoelectric patches because accounting for bending stiffness in these types of structures was significant.

Testing of pressurized thin film toroids has increased significantly in the past few years with renewed interest in inflatable structures. Griffith and Main used a modified impact hammer to excite the global modes of the structure while avoiding local excitation [10]. They found that increasing the internal pressure from 0.8 psi to $1.0 \mathrm{psi}$ in the torus resulted in significantly less damping which they hypothesized was due to a decrease in wrinkling.

Lassiter et. al. tested a torus attached to three struts with a lens in a thermal vacuum chamber [15]. They found significant differences in the response between the structure in ambient and vacuum conditions. The modes did not correlate well and the damping levels were much greater in the ambient test than the vacuum test. They concluded that tests of inflated structures intended for space applications must be performed in a vacuum to obtain meaningful results.

Agnes performed modal tests on an inflated torus using excitation from a 
shaker and a piezoelectric patch. They were unable to clearly identify modes from the test but did demonstrate the viability of piezoelectric excitation of the structure [1] The torus was found to exhibit significant non-linear behavior and the damping was extremely high. The PVDF excitation produced less interference with suspension modes of the free-free torus than excitation from the shaker.

NASA has tested a 5 meter lens, torus, and strut assembly, shown in Figure 1.3. This testing is still ongoing but they have arrived at a number of significant conclusions. A shaker can mass load the test article even if it is attached at a point on a non-inflated part. The physical properties of the structure can change over time, particularly the stiffness of the joints. It is possible to disassemble and reassemble an inflated structure and still have the same dynamic response. This information was received directly from John Lassiter at NASA, the work will be presented at the SDM conference in 2001.

Testing of inflated structures, specifically toroids, is still ongoing. Testing of these structures has proved to be challenging and development of testing procedures is still progressing.

\subsubsection{Piezoelectric Effect}

The Curie brothers originally discovered piezoelectric materials in 1880 [22]. Piezoelectric materials exhibit two useful properties: the direct and converse piezoelectric effects. The direct piezoelectric effect produces a voltage in a material when it is strained, acting as a sensor. The converse piezoelectric effect produces a strain in a material when subjected to a voltage, acting as an actuator. Structures using piezoelectric materials as both sensors and actuators are referred to as "Smart or Intelligent Structures" because of their ability to "sense" and "repair" their condition through control algorithms [22]. As structural requirements become more stringent many engineers are turning to piezoelectric materials to help meet their design requirements.

The linear piezoelectric constitutive equations are written as 


$$
\begin{array}{r}
\mathbf{E}=\beta^{S} \mathbf{D}-h \mathbf{S} \\
\mathbf{T}=c^{D} \mathbf{S}-h^{t} \mathbf{D}
\end{array}
$$

and

$$
\begin{array}{r}
\mathbf{D}=\epsilon^{T} \mathbf{E}+d \mathbf{T} \\
\mathbf{S}=S^{E} \mathbf{T}+d^{t} \mathbf{E}
\end{array}
$$

where $\mathbf{T}, \mathbf{E}, \mathbf{S}$, and $\mathbf{D}$ are the vectors of stress, electric field, strain, and electric displacement (charge per unit area), respectively, $c^{D}$ is the elasticity matrix at constant dielectric displacement, $h$ and $d$ are the piezoelectric constant matrices, $\beta^{S}$ is the dielectric impermeability matrix at constant strain, $s^{E}$ is the elastic compliance matrix at constant electric field, $\epsilon^{T}$ is the dielectric matrix at constant strain, and the superscript $t$ denotes the transpose of a matrix. The electrical and mechanical forces are assumed to balance at all times (quasi-static approximation) to derive equations (1.1), (1.2), (1.3), and (1.4). Equations (1.1) and (1.3) describe the direct piezoelectric effect and equations (1.2) and (1.4) describe the converse piezoelectric effect [22].

A simple way to think of the coupling between the piezo and base structure is shown in Figure 1.4. For a given voltage and stiffness ratio, $k_{s} / k_{a}$, the piezo will output a known force and displacement. The slope of the constant voltage line is determined by the piezoelectric properties of the material. In this 1-D model, the piezo, with stiffness $k_{a}$ is pushing against a spring of stiffness $k_{s}$. Under a constant voltage, if the spring is very stiff, the piezo will output a large force, but little displacement, if the spring is soft, the piezo will output a smaller force but larger displacement.

Piezoelectric devices can be subdivided into two main categories, piezoceramics and piezopolymers. Piezoceramics, such as lead zirconate titanate (PZT) are stiff and brittle materials, whereas piezopolymers, such as polyvinylidene fluoride (PVDF), are highly flexible and tough materials. Piezoceramics generally have a higher electro- 


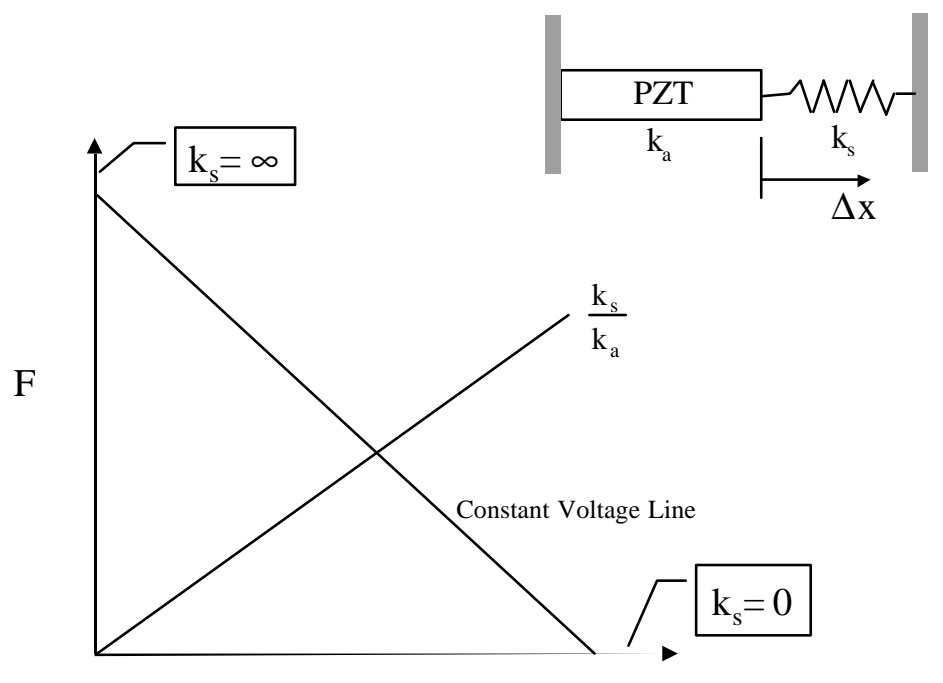

$\Delta \mathrm{x}$

\section{Figure 1.4: 1-D Model of Force Output from a Piezoelectric Device.}

mechanical coupling than piezopolymers so essentially they produce higher voltages per strain and higher strains per voltage.

The piezoelectric effect occurs naturally in quartz crystals, but can be created in other materials. For PVDF, piezoelectricity is induced in the material by stretching the strip and applying a high voltage [22]. The direction of the voltage potential across the strip determines the poling direction of the resulting piezoelectric device. Other materials, such as PZT, require heating to their "Curie Temperature" and then having a voltage applied to them to activate their piezoelectric properties and set the poling direction. The poling direction determines the direction of deformation of the material given the direction of voltage potential.

Dosch et. al. developed a method which allows a single piece of piezoelectric material to simultaneously sense and actuate in a closed loop system. This allows for truly collocated sensors and actuators [5]. The method is realized by using an electrical bridge circuit to measure strain or time rate of strain in the actuator.

The pin-force model is one of the earliest and simplest models to describe the force transferred from an actuator to a substrate. This model treats the actuator 
and substrate as separate bodies connected by pins at the ends of the actuator. The simple pin-force model allows the actuator to expand thereby bending the substrate. It does not account for the bending stiffness of the actuator. This model can be inaccurate when the bending stiffness of the actuator is not significantly less than that of the substrate.

The Bernoulli-Euler model accounts for the bending stiffness of both the actuator and the substrate. Chaudhry and Rogers [3], using "Mechanics Considerations" arrived at the Bernoulli-Euler model from a more "physical" derivation than previous solutions. Their new approach to the model arrived at the same mathematical behavior as the more complicated Bernoulli-Euler model.

\subsubsection{Control Design}

Modern control theory came to the United States from Russia in 1957 after the first launch of Sputnik. The Russians initially developed state space methods and pole placement control techniques, while US engineers were using transfer functions to describe dynamic systems [9]. For a controllable system, using state space methods, it is possible to place the closed loop poles anywhere in the s-plane that the designer desires, if all the states are measured. This means that the closed loop performance can be specified to be anything that the designer wants. The problem with this method is that it is usually not practical to measure all of the states of a system. Using an observer, or state estimator, all of the states do not need to be measured. One disadvantage of a state estimator is that it adds additional dynamics to the system, essentially doubling the number of states.

The largest problem with observers in distributed structural dynamic systems is the concept of observation spillover. A distributed system has an infinite number of modes, however the designer usually only considers some small subset of the modes, usually with the lowest natural frequencies. Observation spillover is the result of the physical measurement of the unmodeled modes. The problem observation spillover can cause instability in the unmodeled modes of the structure. For this reason it is sometimes desirable not to estimate the states. One way to avoid observation spillover 
is to directly use the output of the sensors for feedback control [19].

If the sensors and actuators are collocated the output of the sensor can be fed back directly to the actuator. This method does not allow the designer to place the poles arbitrarily but is simpler than state feedback. Direct output feedback has infinite bandwidth so the effect of the controller on even the high frequency modes can be very significant.

\subsection{Overview of Thesis}

\subsubsection{Contribution}

This thesis investigates the feasibility of using PVDF to attenuate vibration in an inflated torus. The purpose is to develop a predictive model that can be compared with experimental results and will be useful in design. This predictive model is then used to perform an initial study regarding the vibration control of an inflated torus. In addition, the effect of various inflation pressures and aspect ratios on the vibration of the torus is explored.

\subsubsection{Approach}

Chapter 2 describes the modeling approach for the torus. A commercial finite element package, ANSYS, is used to form the mass and stiffness matrices of the pressurized torus. The matrices are then reduced in MATLAB using mass condensation. The eigenvalue problem is then solved for the reduced mass and stiffness matrices and subset of the modes are used to solve for the impulse response of the structure in a state space formulation. Chapter 3 describes the modeling of the interaction between the piezoelectric devices and the torus. Euler-Bernoulli beam theory is used to approximate the behavior of the PVDF bonded to the torus. An observer-based modal state space controller and direct output velocity feedback controller are used to attenuate vibration in the structure. Chapter 4 discusses the effect of torus aspect ratio and pressure on the vibration of the torus. The passive effect of PVDF, considering 
only its mass and stiffness, on the torus is also examined. The effectiveness of each controller is determined by considering the closed loop response of the system to an impulse excitation. 


\section{Chapter 2}

\section{Modeling of a Torus}

\subsection{Introduction}

Analytical solutions for the mode shapes and natural frequencies of a prestressed torus are quite complex. The finite element method provides a relatively easy way to model the system. Commercial finite element programs have become highly developed in the past few years and their utility has increased with the development of faster and faster computers. The analysis method presented here makes use of the availability of highly refined commercial finite element programs to formulate mass and stiffness matrices representing the model. These mass and stiffness matrices can then be manipulated outside of the bounds of the finite element package using MATLAB, a computer mathematical tool specializing in matrix manipulation. The matrices extracted from the finite element model are large and need to be reduced for efficient computation. Mass condensation is performed before solving the eigenvalue problem and modal truncation is used when designing the controller. The model is represented in state space so that traditional control tools can be used to manipulate it. The MAtLAB code to perform this analysis can be found in the Appendix. 


\section{$2.2 \quad$ Finite Element Modeling}

The torus was modeled in ANSYS using linear thin shell elements. Shell93 elements were used that have 8 nodes, four corner nodes and four midside nodes. Each node has 6 degrees of freedom, translation and rotation in $\mathrm{x}, \mathrm{y}$, and $\mathrm{z}$. The rotational inertia is assumed to be negligible for these elements so the rotational mass is zero. The stress field in the pressurized torus is very sensitive to irregularities in the mesh, therefore the mesh was created externally to ANSYS and then the node and element definitions were read in using input files. The MATLAB code to generate the node and element definitions can be found in the Appendix. The model is defined by the geometry, based on the nodes and elements, the real constants, which define the shell element thickness, and the material properties. These values are listed in Table 2.1. The torus is made of Kapton and PVDF patches are attached to it to use as actuators to implement the control algorithms.

Table 2.1: Finite Element Model Material Properties

\begin{tabular}{lll}
\hline \hline Parameter & SI & English \\
\hline Kapton Elastic Modulus & $2.55 \mathrm{e} 9 \mathrm{~Pa}$ & $0.370 \mathrm{e} 6 \mathrm{psi}$ \\
Kapton Mass Density & $1418 \mathrm{~kg} / \mathrm{m}^{3}$ & $1.328 \mathrm{e}-4 \mathrm{lb}^{*} \mathrm{~s}^{2} / \mathrm{in}^{4}$ \\
Kapton Poisson's Ratio & 0.34 & 0.34 \\
Kapton Thickness & $76.2 \mu \mathrm{m}$ & $0.0030 \mathrm{in}$ \\
PVDF Elastic Modulus & $3.0 \mathrm{e} 9 \mathrm{~Pa}$ & $0.435 \mathrm{e} 6 \mathrm{psi}$ \\
PVDF Mass Density & $1728 \mathrm{~kg} / \mathrm{m}^{3}$ & $1.664 \mathrm{e}-4 \mathrm{lb}^{*} \mathrm{~s}^{2} / \mathrm{in}^{4}$ \\
PVDF Poisson's Ratio & 0.33 & 0.33 \\
PVDF Thickness & $28.0 \mu \mathrm{m}$ & $0.0011 \mathrm{in}$ \\
\hline
\end{tabular}

Table 2.2: Finite Element Model Geometric Properties

\begin{tabular}{lll}
\hline \hline Parameter & SI & English \\
\hline Ring Diameter & $15.24 \mathrm{~m}$ & $600 \mathrm{in}$ \\
Tube Diameter & $0.61 \mathrm{~m}$ & $24 \mathrm{in}$ \\
\hline
\end{tabular}




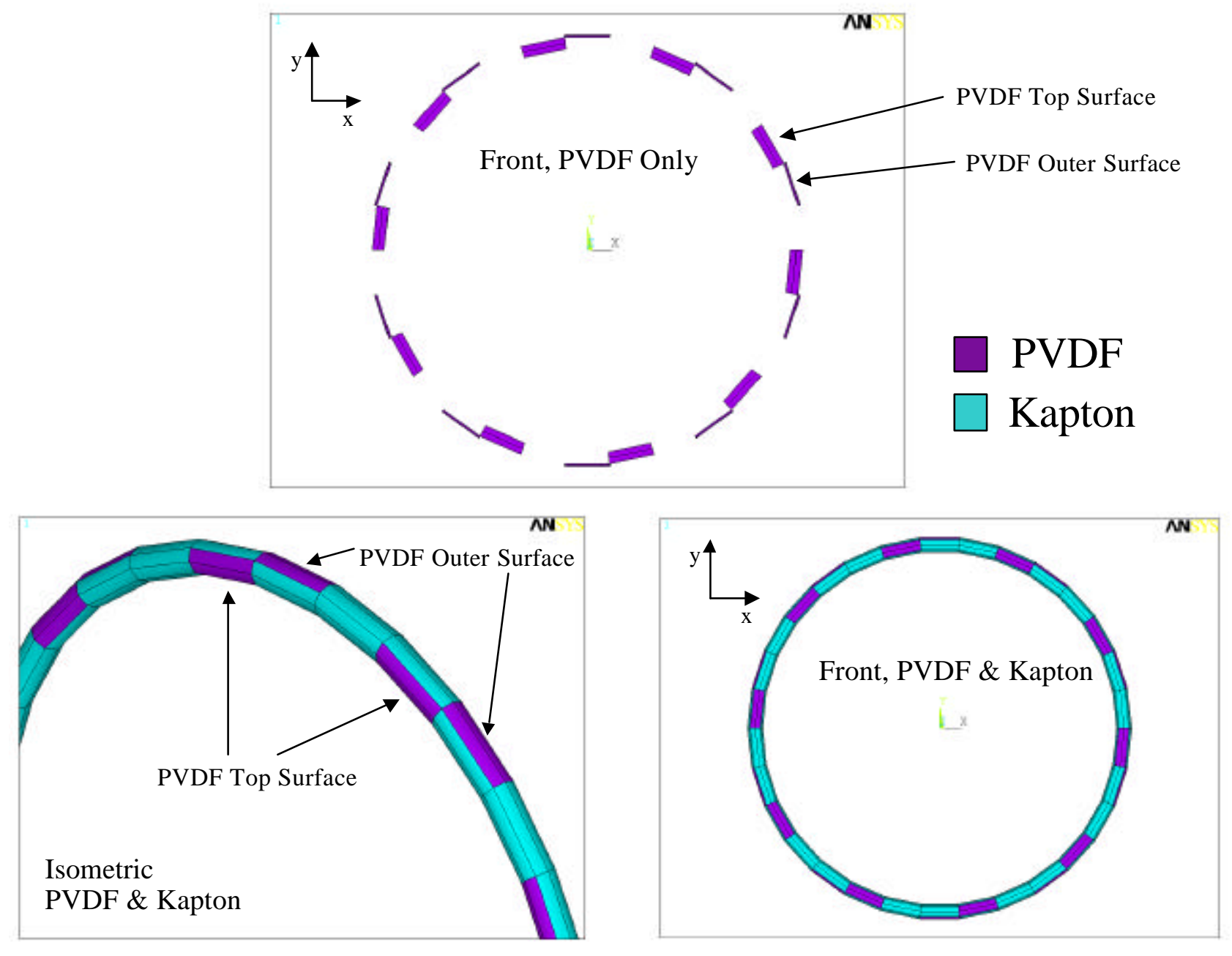

Figure 2.1: Locations of PVDF on Torus.

The PVDF patches were placed on the torus by collocating additional Shell93 elements on top of the existing elements. The location of these patches is shown in Figure 2.1. This is an approximation of the actual structure because the two elements exist in the same plane, thereby having a slightly lower bending stiffness than the actual structure.

An internal pressure of 0.5 psi was applied on all of the Kapton elements to simulate the pressure in torus. The torus was restrained to zero displacement at one node in all degrees of freedom to prevent rigid body motion and a static analysis was performed using the option pstres, on to create a prestressed stiffness matrix to be used in the modal analysis to follow. 
Before performing the modal analysis, the boundary conditions had to be changed. The displacement constraint was removed to solve for the free-free response

of the torus. It was not necessary to remove the internal pressure because ANSYS neglects any applied forces in a modal analysis. It should be noted that some of the solution options were modified for better convergence. The subspace working size (subsiz), number of extra vectors (npad), and number of modes per memory block (nperbk) were all doubled from their default values for better convergence. This seemed to be necessary due to the first six frequencies being zero, the rigid body modes, and the fact that most of the modes came in orthogonal pairs at the same frequency.

\subsubsection{Mass and Stiffness Extraction}

The information defining the mass and stiffness matrices is defined in the file jobname.full created by ANSYS after performing a modal analysis. This file contains the information in binary format that needs to be converted and extracted from the file. This procedure is described in the Guide to Interfacing with ANSYS. It should be noted for anyone who is interested in doing this that the process is not trivial. It requires the user to have both the Microsoft Visual $\mathrm{C}++$ compiler and the Compaq Visual Fortran compiler of the specific version referenced in the ANSYS Installation and Configuration Guide. To compile the source code rdfull.f which reads the mass and stiffness matrices the user first needs to rename the source code rdfull.f to userprog.f. The user then needs to run the ANSYS program rdrwrt.bat with the option userprog from a DOS window, the full command is rdrwrt userprog. This produces the executable userprog.exe which is used to extract the mass and stiffness matrices from the file jobname.full by renaming the file jobname.full to file.full and then executing userprog.exe in the directory where file.full resides. It should be noted that the author never got this procedure to fully work under Windows98, he could never compile the source code userprog.f. The author believes the root of the problem to be that the Windows98 environment seems to have problems setting environment variables from batch files. He has been assured that the procedure does work on a 
WindowsNT platform. Eventually he got a compiled version of the file userprog.exe from the ANSYS support representative and just used that. However, to run that file the user still needs to have the Compaq Visual Fortran compiler installed on the computer system.

The output from running the program userprog.exe in the same directory as the file file.full is two files, MASS.MATRIX and STIFFNESS.MATRIX. These files consist of three columns of numbers representing the row number, column number, and nonzero value of the matrix entry. They only contain the upper diagonal portion of the matrix. The model degrees of freedom are ordered by node number and nodal degree of freedom. For instance, for a two node model with six degrees of freedom per node, the model degrees of freedom are represented by

$$
x=\left[\begin{array}{c}
x 1 \\
y 1 \\
z 1 \\
\operatorname{rot} x 1 \\
\operatorname{rot} y 1 \\
\operatorname{rot} z 1 \\
x 2 \\
y 2 \\
z 2 \\
\operatorname{rot} x 2 \\
\operatorname{rot} y 2 \\
\operatorname{rot} z 2
\end{array}\right]
$$

\subsection{Finite Element Model Verification}

A number of test cases are examined to verify the use of the commercial finite element program ANSYS to model the dynamics of prestressed structures. First a tensioned string and tensioned membrane are compared to analytical solutions. Then convergence is demonstrated on the model of the torus by solving for the modal frequencies 
Table 2.3: Properties of String

\begin{tabular}{ll}
\hline \hline Parameter & Value \\
\hline Elastic Modulus & $30 \mathrm{e} 6 \mathrm{lb} / \mathrm{in}^{2}$ \\
Volume Density & $0.285 \mathrm{lb}^{*} \mathrm{~s}^{2} / \mathrm{in}^{4}$ \\
X-section & $0.001 \mathrm{in}^{2}$ \\
$l$ & $1 \mathrm{in}$ \\
$\tau$ & $1 \mathrm{lb}$ \\
\hline
\end{tabular}

with varying mesh densities. Finally the converged torus model is compared with a model proposed by Liepins [17] to solve for the axisymmetric modes of vibration.

\subsubsection{Tensioned String}

A tensioned string is the 1-D analogy of a 2-D membrane. To initially verify the capabilities of ANSYS, a tensioned string was modeled. The analytical calculation of the natural frequencies of a tensioned string is well documented [12]. The natural frequencies of the tensioned spring are expressed as

$$
\sigma_{n}=\frac{n}{2 l} \sqrt{\frac{\tau}{\rho_{l}}}
$$

where $\sigma_{n}$ are the natural frequencies in $\mathrm{Hz}$ of index $n, l$ is the length of the string in inches, $t$ is the string tension in lbs, and $\rho_{l}$ is the density per unit length of the string in $\mathrm{lb}^{*} \mathrm{~s}^{2} / \mathrm{in}^{2}$. The string was meshed using LINK8 elements. First a static analysis was performed with a unit force applied to the string on one end and the other end fixed. This analysis solved for the stiffness of the string under tension. A modal analysis could then be performed using the "prestressed" stiffness matrix solved for in the static analysis. For the modal analysis the string was supported at both ends and the force was removed. Meshes of 10,20, and 30 elements were solved to examine the effect of mesh density on the results. The material properties used are listed in Table 2.3. 
Table 2.4: First Three Modes of Vibration of a String in Tension

\begin{tabular}{|c|r|r|r|r|r|r|r|}
\hline & Analytical & \multicolumn{2}{|c|}{10 Elems } & \multicolumn{2}{c|}{20 Elems } & \multicolumn{2}{c|}{30 Elems } \\
\hline Modes & Frequency & Frequency & Error & Frequency & Error & Frequency & Error \\
\hline 1 & 29.617 & 29.739 & $0.41 \%$ & 29.648 & $0.10 \%$ & 29.631 & $0.05 \%$ \\
2 & 59.235 & 60.23 & $1.68 \%$ & 59.479 & $0.41 \%$ & 59.343 & $0.18 \%$ \\
3 & 88.852 & 92.166 & $3.73 \%$ & 89.677 & $0.93 \%$ & 89.218 & $0.41 \%$ \\
\hline
\end{tabular}

The finite element results were found to correlate very well with the analytical solution. The accuracy between the modal frequencies decreased as the mode number increased. Increasing numbers of elements increased the accuracy of the solution. As the number of elements increased, the solution converged to the analytical solution. Table 2.4 summarizes the results of the analytical and finite element models and compares the error between them.

\subsubsection{Tensioned Membrane}

A Kapton coupon was modeled in ANSYS and compared to the analytical results obtained by Williams [24]. Williams considered the non-uniformly tensioned coupon to be a membrane, therefore the coupon had no bending stiffness. Williams found the natural frequency of a rectangular coupon, shown in Figure 2.2, to be

$$
\omega_{m n}=\pi \sqrt{\frac{P_{1}}{\rho}\left(\left(\frac{m}{a}\right)^{2}+\square\left(\frac{n}{b}\right)^{2}\right)}
$$

The values $P_{1}$ and $P_{2}$, where $P_{2}=P_{1} \square$, represent the force per unit length on the membrane, the values $a$ and $b$ are the dimensions of the membrane, and $m$ and $n$ are the modal indices. The case used for comparison is shown in Table 2.5.

A static solution of the stretched membrane was first performed in ANSYS with the boundary conditions illustrated in Figure 2.3a to produce the "prestressed" stiffness matrix. The "prestressed" stiffness matrix was then used to solve for the 

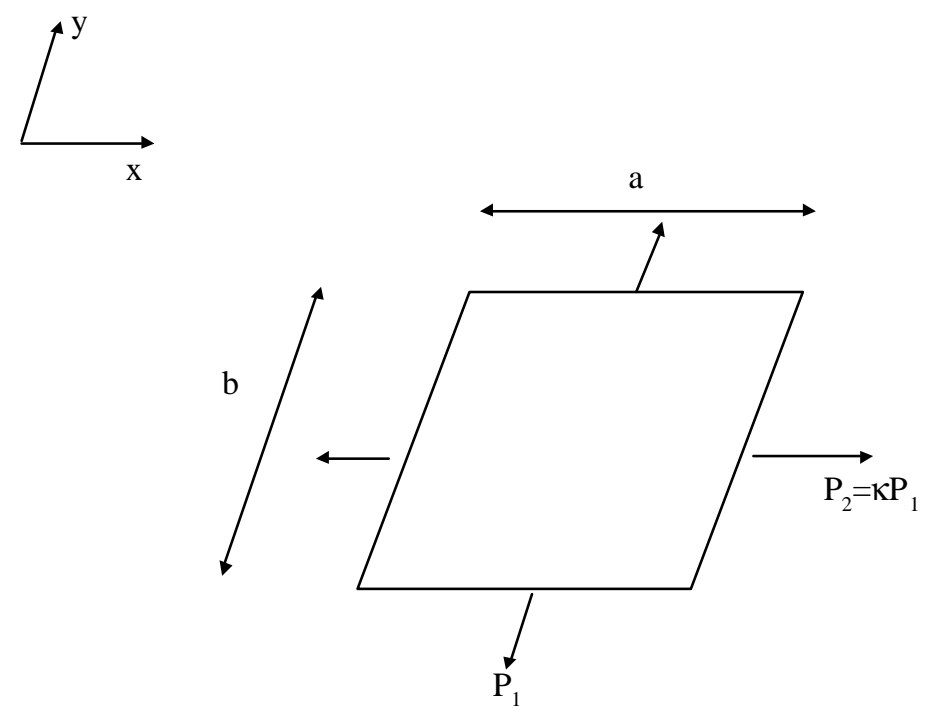

Figure 2.2: Membrane Coupon Under Non-Uniform Tension $P_{1}$ and $P_{2}$

Table 2.5: Properties of Membrane

\begin{tabular}{ll}
\hline \hline Parameter & Value \\
\hline$P_{1}$ & $0.75 \mathrm{lb} / \mathrm{in}$ \\
$\square$ & 2 \\
$\rho$ & $3.98 \mathrm{e}-7 \mathrm{lb} \mathrm{s} / \mathrm{in}^{3}$ \\
$a$ & $5.0 \mathrm{in}$ \\
$b$ & $2.5 \mathrm{in}$ \\
$E$ & $0.37 \mathrm{e} 6 \mathrm{lb} / \mathrm{in}^{2}$ \\
\hline
\end{tabular}




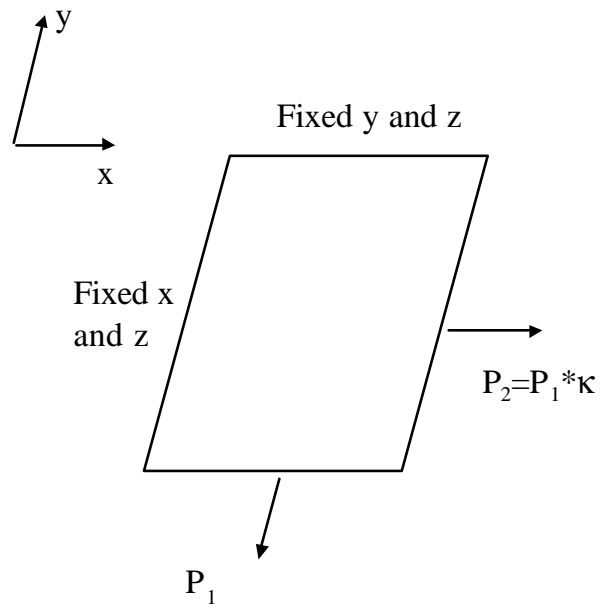

(a)

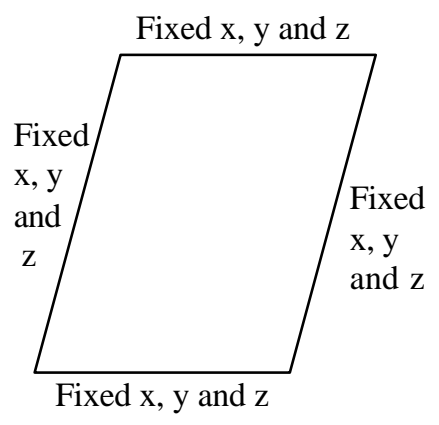

(b)

\section{Figure 2.3: (a) Boundary Conditions for Static Analysis (b) Boundary Conditions for Dynamic Analysis}

first 20 modes of the structure. The boundary conditions used for the modal analysis are shown in Figure 2.3b.

The analytical and finite element models agreed very well. The error was less than one percent for the first 19 modes. The analytical and finite element calculations of natural frequency, and the error between them, are shown in Table 2.6.

It is interesting to note that the error was related not to the frequency but to the highest modal index. For instance, the 4,2 mode had a lower error than the 6,1 mode even though the 4,2 mode had a higher frequency. This is expected because the larger number of elements in the long dimension could more accurately represent the curvature of the membrane at higher order modes in the long dimension than the smaller number of elements in the short dimension. This allowed for a more accurate result of higher order modes in the long dimension than the short. For instance the error in the $m=3, n=1$ mode is much less than the $m=1, n=3$ mode. 
Table 2.6: Analytical and Finite Element Natural Frequencies of Tensioned Membrane.

\begin{tabular}{|c|r|r|r|r|r|r|}
\hline & & & \multicolumn{2}{|c|}{ Analytical } & \multicolumn{2}{|c|}{ Finite Element } \\
\hline Modes & $m$ & $n$ & $w_{m n}$ & $f_{m n}, \mathrm{~Hz}$ & $f, \mathrm{~Hz}$ & Error \\
\hline 1 & 1 & 1 & 2586.58 & 411.67 & 412.07 & $0.098 \%$ \\
2 & 2 & 1 & 2986.73 & 475.35 & 475.84 & $0.102 \%$ \\
3 & 3 & 1 & 3554.92 & 565.78 & 566.62 & $0.148 \%$ \\
4 & 4 & 1 & 4223.87 & 672.25 & 673.83 & $0.235 \%$ \\
5 & 5 & 1 & 4952.93 & 788.28 & 790.6 & $0.294 \%$ \\
6 & 1 & 2 & 4952.93 & 788.28 & 791.17 & $0.366 \%$ \\
7 & 2 & 2 & 5173.17 & 823.34 & 825.63 & $0.279 \%$ \\
8 & 3 & 2 & 5520.74 & 878.65 & 881.08 & $0.276 \%$ \\
9 & 6 & 1 & 5719.15 & 910.23 & 915.1 & $0.535 \%$ \\
10 & 4 & 2 & 5973.46 & 950.71 & 953.57 & $0.301 \%$ \\
11 & 5 & 2 & 6509.43 & 1036.01 & 1039.8 & $0.366 \%$ \\
12 & 7 & 1 & 6509.43 & 1036.01 & 1043.7 & $0.743 \%$ \\
13 & 6 & 2 & 7109.84 & 1131.57 & 1137 & $0.480 \%$ \\
14 & 8 & 1 & 7315.96 & 1164.37 & 1175.8 & $0.981 \%$ \\
15 & 1 & 3 & 7366.59 & 1172.43 & 1179.7 & $0.620 \%$ \\
16 & 2 & 3 & 7516.44 & 1196.28 & 1203.54 & $0.607 \%$ \\
17 & 3 & 3 & 7759.75 & 1235.00 & 1242.2 & $0.583 \%$ \\
18 & 7 & 2 & 7759.75 & 1235.00 & 1242.8 & $0.631 \%$ \\
19 & 4 & 3 & 8088.10 & 1287.26 & 1294.6 & $0.570 \%$ \\
20 & 9 & 1 & 8133.93 & 1294.55 & 1310.9 & $1.263 \%$ \\
\hline
\end{tabular}


Table 2.7: Torus Model Convergence at .5 psi.

\begin{tabular}{|r|r|r|r|r|r|r|}
\hline & \multicolumn{6}{|c|}{ Frequencies by Number of Elements } \\
\hline Modes & $15 \times 4$ & $15 \times 8$ & $30 \times 4$ & $30 \times 8$ & $60 \times 4$ & $60 \times 8$ \\
\hline Mode 1 & 3.4025 & 3.40622 & 3.3985 & 3.40565 & 3.39909 & 3.40587 \\
Mode 2 & 4.99523 & 5.00407 & 4.9971 & 5.00356 & 4.99825 & 5.00304 \\
Mode 3 & 7.70049 & 7.6692 & 7.63148 & 7.65431 & 7.63221 & 7.65413 \\
Mode 4 & 8.7249 & 8.77126 & 8.7228 & 8.76352 & 8.7247 & 8.7618 \\
Mode 5 & 13.30876 & 11.95952 & 12.94873 & 12.99909 & 12.94626 & 12.99655 \\
Mode 6 & 13.74654 & 13.08892 & 13.68895 & 13.75992 & 13.69251 & 13.75508 \\
Mode 7 & 18.9829 & 13.81573 & 19.38329 & 19.4779 & 19.36662 & 19.46825 \\
\hline
\end{tabular}

\subsubsection{Model Convergence}

To confirm the convergence of the model, the torus was meshed with 120, 240, and 480 elements. Table 2.7 shows the resulting first 7 natural frequencies for each model. The heading "NNxN" refers to the mesh density of the model, where " $30 \mathrm{x} 4$ " is a torus meshed with 30 elements around the ring and four elements around the tube for a total of 120 elements.

The models with only 15 elements around the ring do a poor job of predicting modes higher than 4 . All of the models with 30 elements or greater around the ring produce very similar results. There is only a $0.44 \%$, or $0.08496 \mathrm{~Hz}$, difference in modal frequency at the seventh mode between the $60 \times 8$ and the $30 \times 4$ models. The 30x8 model will be used for the remainder of this analysis. Eight elements are necessary around the tube to produce fine enough discretization to locate the PVDF excitation.

\subsubsection{External Verification}

Modal testing of inflated structures has proved challenging. At present there is no reliable published data on modal testing of inflated toroids. For this reason the finite element model will be verified from previous analytical work available in the literature.

The model results were compared with Liepins work in 1965 [17]. Liepins ex- 
Table 2.8: Verification Model Properties

\begin{tabular}{ll}
\hline Property & Value \\
\hline Major Radius & $1 "$ \\
Minor Radius & $.75 "$ \\
Thickness & $.0075 "$ \\
Young's Modulus & $0.37 \mathrm{e} 6 \mathrm{psi}$ \\
Density & $0.13276 \mathrm{e}-3 \mathrm{lb}^{*} \mathrm{~s}^{2} / \mathrm{in}^{4}$ \\
Internal Pressure & $7.4 \mathrm{psi}$ \\
\hline
\end{tabular}

Table 2.9: Comparison of FEA and Liepins Model

\begin{tabular}{lll}
\hline \multicolumn{3}{c}{ Frequencies in $\mathrm{Hz}$} \\
\hline Liepins & FEA & Error \\
958 & 979 & $2.42 \%$ \\
3555 & 3516 & $1.09 \%$ \\
3905 & 3877 & $0.72 \%$ \\
3985 & 3906 & $0.64 \%$ \\
\hline
\end{tabular}

amined the axisymmetric modes of the prestressed torus using Sanders linear shell theory and finite difference methods. The material properties and geometry examined is listed in Table 2.8. Liepins was concerned with the axisymmetric modes of vibration, which is only a small subset of the actual modes of the torus. The finite element solution produces a much larger number of modes and it is necessary to sort though them to find the axisymmetric modes to match those calculated by Liepins. The first four axisymmetric modes of vibration are compared.

The models were found to compare very well as shown in Table 2.9. The finite element model had 30 elements around the tube diameter and 80 elements around the ring diameter. 


\subsection{Problem Formulation}

The dynamics of the torus can be represented by Newton's second law, $F=m a$. Summing forces on the torus results in the matrix equation

$$
\begin{aligned}
& M \ddot{x}+K x=f \\
& y=C x
\end{aligned}
$$

where $M$ and $K$ represent the symmetric mass and stiffness matrices, $x$ and $\ddot{x}$ are the vectors of displacement and acceleration, and $f$ is a vector of external disturbances. The vector $y$ is the desired output from the system. The mass and stiffness matrices are initially very large, on the order of 4000 by 4000, and can be condensed using mass condensation. These matrices need to be reduced in order to solve the eigenvalue problem to determine the natural frequencies and modes of vibration. Without reducing the number of degrees of freedom in the system, MATLAB was unable to solve for the eigenvalues and eigenvectors. In addition, because the rotational inertia of the shells is neglected, there are zeros on the diagonal of the mass matrix. These massless degrees of freedom need to be removed for many solution techniques to work. The MATLAB code used to perform the following modeling steps can be found in the Appendix.

\subsubsection{Mass Condensation}

Mass condensation is essentially a specific case of Guyan reduction where only massless degrees of freedom are removed [12]. Guyan reduction is a common model reduction technique which is defined in many texts, for example, Inman 1996. In this case

all of the rotational degrees of freedom are massless. The Shell93 elements used in the finite element analysis assume that the rotational inertia is negligible in the model and so the rotational masses are zero, therefore they can be removed. Mass condensation, or Guyan reduction, is characterized by separating the mass and stiffness matrices into significant and insignificant degrees of freedom, $x_{1}$ and $x_{2}$ respectively. This results in the matrix equation 


$$
\left[\begin{array}{ll}
M_{11} & M_{12} \\
M_{21} & M_{22}
\end{array}\right]\left[\begin{array}{c}
\ddot{x}_{1} \\
\ddot{x}_{2}
\end{array}\right]+\left[\begin{array}{ll}
K_{11} & K_{12} \\
K_{21} & K_{22}
\end{array}\right]\left[\begin{array}{l}
x_{1} \\
x_{2}
\end{array}\right]=\left[\begin{array}{l}
f_{1} \\
f_{2}
\end{array}\right]
$$

The method of Guyan reduction leads to the coordinate transformation matrix $Q$. This coordinate transformation reduces the full coordinate system $x$ to the reduced coordinates $x_{1}$. The coordinate transformation is defined by

$$
x=Q x_{1}
$$

where

$$
Q=\left[\begin{array}{c}
I \\
-K_{22}^{-1} K_{21}
\end{array}\right]
$$

So if equation (2.6) is premultiplied by $Q^{T}$ the reduced order system is represented by

$$
\bar{M} \ddot{x}_{1}+\bar{K} x_{1}=Q^{T} f
$$

where $\bar{M}=Q^{T} M Q$ and $\bar{K}=Q^{T} K Q$. Now having a reduced order system the eigenvalue problem can be performed to solve for the eigenvalues and eigenvectors of the system.

\subsubsection{Eigenvalue Solution}

The eigenvalue problem is defined such that for a matrix $X, X \Phi=\Phi \Lambda$, where $\Lambda$ is a diagonal matrix of eigenvalues and $\Phi$ is a matrix of eigenvectors. Choleski decomposition is used to transform the equation of motion, equation (2.9) in this case, to a symmetric eigenvalue problem. Choleski decomposition is defined by the equation 


$$
U^{T} U=\bar{M}
$$

This equation leads us to the coordinate transformation defined as

$$
x_{1}=U^{-1} q
$$

Applying the coordinate transformation to equation (2.9) we get the following equations

$$
\begin{gathered}
U^{T} U \ddot{x_{1}}+\bar{K} x_{1}=Q^{T} f \\
U^{T} \ddot{q}+\bar{K} U^{-1} q=Q^{T} f \\
\ddot{q}+U^{-T} \bar{K} U^{-1}=U^{-T} Q^{T} f \\
\tilde{K}=U^{-T} \bar{K} U^{-1}
\end{gathered}
$$

Solving the eigenvalue problem for $\tilde{K}$ produces the matrices $\Lambda$ and $\Phi$, the eigenvalues and eigenvectors respectively.

The eigenvalue problem was solved using the MATLAB eigs function. This function allows the user to request the number and range of eigenvalues. In this case it was desirable to get the first few lowest eigenvalues and their corresponding eigenvectors. It should be recognized that this produces a non-square matrix of eigenvectors. The eigenvectors have been transformed with two prior coordinate transformations as defined by equations (2.7) and (2.11). To obtain the physical mode shapes it is necessary to reverse the coordinate transformations on the eigenvectors by

$$
\bar{\Psi}=Q U^{-1} \Phi
$$

These physical mode shapes are not mass normalized due to the mass condensation. The coordinate transformation $U^{-1} \Phi$ produces mass normalized transformed 
mode shapes, but then the transformation back to physical coordinates from the mass condensation coordinates removes the mass normalization. In other words if the mass condensation had not been performed you would have the mass normalized mode shapes at this point. The physical mode shapes are mass normalized by

$$
\begin{gathered}
\bar{\Psi}=\left[\bar{\Psi}_{1}, \bar{\Psi}_{2}, \ldots, \bar{\Psi}_{n}\right] \\
m_{i}=\bar{\Psi}_{i}^{T} M \bar{\Psi}_{i} \\
\Psi_{i}=\frac{1}{\sqrt{m_{i}}} \bar{\Psi}_{i} \\
\Psi=\left[\Psi_{1}, \Psi_{2}, \ldots, \Psi_{n}\right]
\end{gathered}
$$

\subsubsection{Modal Equations}

The equation of motion, equation (2.5), can now be expressed in modal coordinates, through the modal transformation

$$
x=\Psi r
$$

as

$$
\begin{aligned}
& I \ddot{r}+\Lambda r=\Psi^{T} f \\
& \quad y=C \Psi r
\end{aligned}
$$

by recognizing that

$$
\begin{aligned}
\Psi^{T} M \Psi & =I \\
\Psi^{T} K \Psi & =\Lambda
\end{aligned}
$$

because the eigenvectors are mass normalized. The modal equation consists of square matrices. The matrix $\Lambda$ is a diagonal matrix of natural frequency, in $\mathrm{rad} / \mathrm{s}$, squared. The rigid body modes were removed from the mode shape matrix $\Psi$ for a clearer understanding of the behavior of the structure [12]. 


\subsubsection{State Space Formulation}

The modal equation was then reduced to first order by the transformation

$$
\begin{aligned}
& q_{1}=r \\
& q_{2}=\dot{r}
\end{aligned}
$$

yielding

$$
\left[\begin{array}{c}
\dot{q}_{1} \\
\dot{q}_{2}
\end{array}\right]=\left[\begin{array}{cc}
0 & 1 \\
-\Lambda & 0
\end{array}\right]\left[\begin{array}{c}
q_{1} \\
q_{2}
\end{array}\right]+\left[\begin{array}{c}
0 \\
\Psi^{T} B
\end{array}\right] f
$$

where $f$ is now a horizontal vector of $n$ inputs and $B$ is a matrix with $n$ columns. The product $B f$ is equivalent to the vector $f$ used previously, as in equation (2.5).

Equation (2.27) considers no damping. Modal damping can be added in the form $2 \zeta \omega_{n}$ where $\omega_{n}$ is the modal frequency and $\zeta$ is the damping ratio. In this case both $\omega_{n}$ and $\zeta$ are matrices. The matrix of modal frequencies can be expressed as $\sqrt{\Lambda}$ and the matrix of damping ratios is a diagonal matrix of the individual modal damping ratios. A modal damping ratio of 0.01 will be used here. Therefore, adding damping results in the equation

$$
\left[\begin{array}{l}
\dot{q}_{1} \\
\dot{q}_{2}
\end{array}\right]=\left[\begin{array}{cc}
0 & 1 \\
-\Lambda & -2 \zeta \sqrt{\Lambda}
\end{array}\right]\left[\begin{array}{c}
q_{1} \\
q_{2}
\end{array}\right]+\left[\begin{array}{c}
0 \\
\Psi^{T} B
\end{array}\right] f
$$

Equations (2.29) and (2.28) can now be expressed in compact form as

$$
\begin{array}{r}
\dot{q}=\bar{A} q+\bar{B} f \\
\\
y=\bar{C} q
\end{array}
$$

Using this form a controller can now be designed. 


\subsection{Summary}

At this point a state space model has been developed to describe the dynamics of the free-free torus. This representation is in modal space and the response is based upon the summation of a truncated set of the modes of vibration, indirectly the eigenvectors, of the system. The modal frequencies are also solved, represented by the square root of the eigenvalues. The MATLAB code is validated by comparing the natural frequencies and mode shapes as calculated by MATLAB to those calculated by ANSYS, they are found to match to a minimum of four decimal places. The next chapter will derive a model for determining the force transferred to the torus by the PVDF and will then examine two control methods for attenuating vibration in the torus. 


\section{Chapter 3}

\section{Actuators and Control Design}

\subsection{Introduction}

To attenuate vibration in the torus, a closed loop control design is implemented. Piezoelectric patches, made of PVDF, are bonded to the surface of the torus and act as both sensors and actuators. This chapter will discuss the modeling of the actuators and the design of the control system. The bonded piezoelectric patches are treated as layered beams to determine the force output to the torus. Two control techniques are examined. Pole placement techniques are initially chosen as a one of the most basic benchmark state space control techniques. Velocity feedback is examined as a simpler control algorithm. Optimal control is briefly discussed as a better alternative for choosing gain matrices in multi-input systems. None of the controllers designed here are likely ideal. The controllers chosen here are for the purpose of demonstrating the controllability of the system, not necessarily because they are the best controllers to use.

\subsection{Piezoelectric Modeling}

One of the simplest models of actuator-substrate interaction is the pin-force method. This method treats the substrate as a beam and the actuator as an expanding rod with their ends pinned. When the actuator expands it produces a moment on the 
substrate thereby bending it. This model can be highly inaccurate because it does not consider the bending stiffness of the actuator. The Bernoulli-Euler model does account for the bending stiffness of the actuator.

To determine the force transferred to the torus from the piezoelectric patches, Bernoulli-Euler beam theory is used. This method treats the actuator and substrate as a composite beam. The strain at the interface between the actuator and substrate is equal, using the assumption of perfect bonding. This assumption leads us to the following moment equation derived by Chaudhry and Rogers [3].

$$
M=-\left(E_{a} I_{a}+E_{b} I_{b}\right) k
$$

where $k$ is the curvature of the beam and $I_{a}$ and $I_{b}$ are the moment of inertia of the actuator and beam respectively. These values are then expressed by

$$
\begin{gathered}
k=\frac{6}{t_{b}} \frac{T(1+T)}{6 T+4 T^{2}+4+\Psi T^{2}+\Psi^{-1}} \Delta \\
I_{a}=\frac{1}{12} t_{a}^{3} b+t_{a} b\left(z-\frac{t a}{2}\right)^{2} \\
I_{b}=\frac{1}{12} t_{b}^{3} b+t_{b} b\left(t_{a}+\frac{t_{b}}{2}-z\right)^{2} \\
\Delta=d_{31} E_{3} \\
T=\frac{t_{b}}{t_{a}} \\
\Psi=\frac{E_{b} t_{b}}{E_{a} t_{a}} \\
z=\frac{\frac{t_{a}}{2} t_{a} \frac{E_{a}}{E_{b}}+\left(t_{a}+\frac{t_{b}}{2}\right) t_{b}}{t_{a} \frac{E_{a}}{E_{b}}+t_{b}}
\end{gathered}
$$

The physical constants are defined in Table 3.1.

The force transferred to the beam, $M$, is in the form of two opposing moments at the ends of the piezo. If the piezo is used as a sensor, it measures the difference in the rotations between the ends of the piezo.

The Bernoulli-Euler model is obviously a rough approximation of the actual structure. This model assumes a pinned-pinned beam in pure bending. The actual 
Table 3.1: Variable Definitions and Values for Bernoulli-Euler Beam Model

\begin{tabular}{lll}
\hline \hline Parameter & Variable & Value \\
\hline PVDF Elastic Modulus & $E_{a}$ & $3.0 \mathrm{e} 9 \mathrm{~Pa}$ \\
PVDF Thickness & $t_{a}$ & $28 \mu \mathrm{m}$ \\
Kapton Elastic Modulus & $E_{b}$ & $2.55 \mathrm{e} 9 \mathrm{~Pa}$ \\
Kapton Thickness & $t_{b}$ & $76.2 \mu \mathrm{m}$ \\
Piezoelectric Constant & $d_{31}$ & $23 \mathrm{e}-12 \mathrm{~m} / \mathrm{V}$ \\
Width of PVDF Patch & $b$ & $.0245 \mathrm{~m}$ \\
Length of PVDF Patch & $l$ & $.0726 \mathrm{~m}$ \\
Electric Field Max & $E_{3}$ & $30 \mathrm{~V} / \mu \mathrm{m} \mathrm{DC}$ \\
\hline
\end{tabular}

structure is a curved shell with a piezoelectric patch covering only a portion of it. This simplification produces two questions. Is it appropriate to model the force transferred from the PVDF to the Kapton as two opposing moments? Is the calculation of maximum possible force generated by the PVDF accurate? The answer to these two questions would better determine the validity of this model.

\subsection{Control Design}

Two different control approaches will be investigated for the control system design. First pole placement techniques, using full state feedback and then adding an observer, are examined. These techniques present practical problems, such as observation spillover. A simpler control method, velocity feedback, is then explored to determine its effectiveness. Velocity feedback also has limitations. An observer can be designed to roll off at high frequencies but velocity feedback control does not roll off, so it can have a significant impact on higher order modes. The problem of observation spillover will be examined to determine its effect on the system.

\subsubsection{Actuator Placement}

Twenty PVDF piezopolymer actuators are used to control the structure. Ten actuators on the outer surface control in-plane modes and ten actuators on the top surface 
control out-of-plane modes. The location and number of these actuators has been chosen somewhat arbitrarily. The piezos used as actuators are also used as sensors on the model. This can be accomplished through the process developed by Dosch, Inman, and Garcia [5]. Using this process produces collocated sensors and actuators.

The force from the actuator influences the system through the control input matrix $B$ in equation (2.29). For a system with twenty actuators $B$ has dimensions of 10x20, considering ten modes. The actuators are assumed to bend only on their primary axis. The actuators on the outer surface create force in only the rotz (rotation about the $z$ axis) direction. The actuators on the top surface create force in both the rotx and roty direction depending on their orientation. See Figure 2.1 for a definition of the coordinate system.

Consider the example of a four node system with rotations in $x, y$, and $z$ at each node. The first two nodes represent a piezo on the top of the torus and the second two nodes represent a piezo on the bottom of the torus. The angle $\theta$ is the angle from the $x$ axis, in the $x y$-plane to the center of the piezo. For this case the control input matrix $B$ is

$$
B=\left[\begin{array}{cc}
1 & 0 \\
0 & 0 \\
0 & 0 \\
-1 & 0 \\
0 & 0 \\
0 & 0 \\
0 & \operatorname{Cos}(\theta) \\
0 & \operatorname{Sin}(\theta) \\
0 & 0 \\
0 & -\operatorname{Cos}(\theta) \\
0 & -\operatorname{Sin}(\theta) \\
0 & 0
\end{array}\right]
$$

where the states are 


$$
\left[\begin{array}{l}
\operatorname{rot} x 1 \\
\operatorname{roty} 1 \\
\operatorname{rot} z 1 \\
\operatorname{rot} x 2 \\
\operatorname{roty} 2 \\
\operatorname{rot} z 2 \\
\operatorname{rotx} 3 \\
\operatorname{roty} 3 \\
\operatorname{rot} z 3 \\
\operatorname{rot} x 4 \\
\operatorname{roty} 4 \\
\operatorname{rot} z 4
\end{array}\right]
$$

Controllability, in very loose terms, essentially means that all of the states can be influenced by the input. To place the system poles arbitrarily using full state feedback a system must be controllable. Using twenty actuators on the torus, the system can be shown to be controllable through the algebraic controllability condition

$$
\left[\begin{array}{lllll}
\bar{B} & \bar{A} \bar{B} & \bar{A}^{2} \bar{B} & \ldots & \bar{A}^{k-1} \bar{B}
\end{array}\right]
$$

where the rank of this matrix equals $k$, the number of states, in this case twenty. The matrices $\bar{A}$ and $\bar{B}$ are defined by equations (2.29) and (2.30). It should be noted that 20 actuators are not necessary to create a controllable system, less may be used.

There is a small amount of coupling between in-plane and out-of-plane modes. The coupling is induced by placement of the actuators, which results in slightly nonsymmetric mass loading of the torus. The actual structure will exhibit some coupling due to small asymmetries in the torus, as well as the asymmetries caused by the location of the actuators. The small asymmetries in the torus are due to manufacturing variability. 


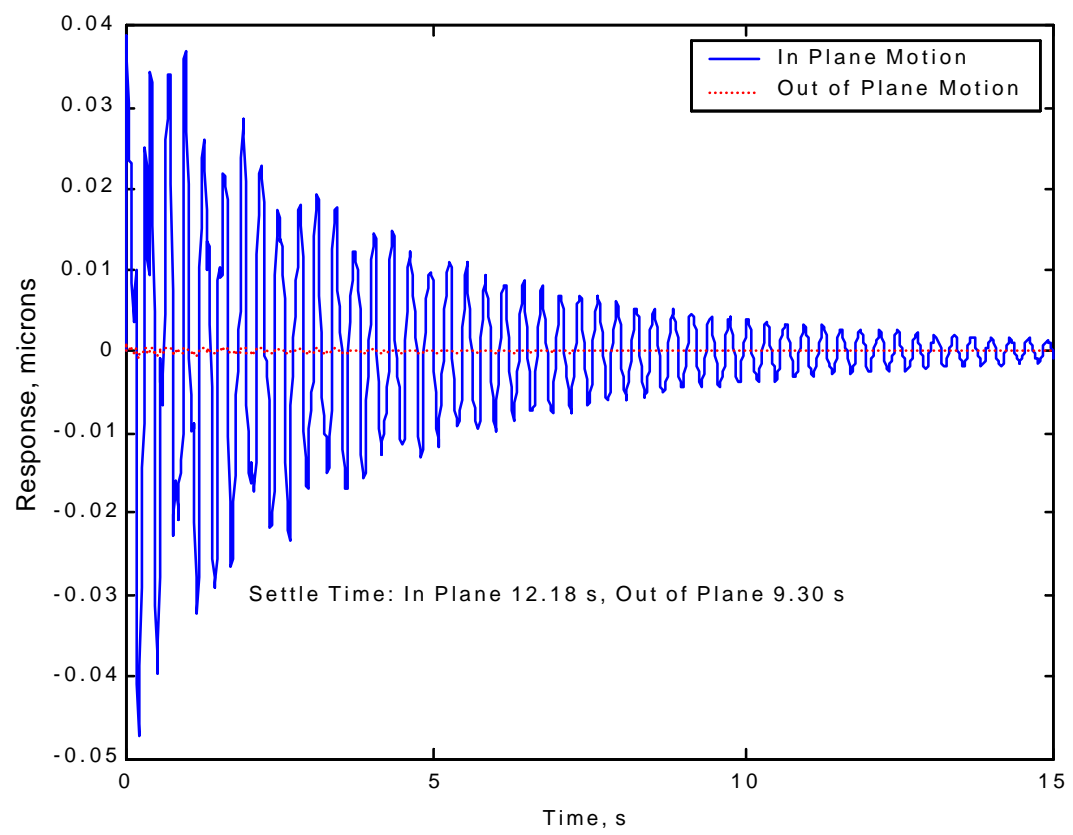

Figure 3.1: Open Loop Impulse Response to In-Plane Excitation.

This phenomenon can be seen more clearly by examining the impulse response of the torus to an in-plane load, shown in Figure 3.1. The impulse applied was 4.4e-6 $\mathrm{N}^{*} \mathrm{~s}\left(1 \mathrm{e}-6 \mathrm{lbf}^{*} \mathrm{~s}\right)$. Clearly there is a significant in-plane response, however there is also a small out-of-plane response.

\subsubsection{Full State Feedback Modal Controller}

Full state feedback control is not very practical. It requires the measurement of every state in the model. This is not really possible in this system, however an examination of full state feedback should give some insight into the problem and acts as a natural precursor to discussion of state observers.

The full state feedback controller will be implemented in modal space to help reduce the order of the model. Only the first ten modes will be considered. It is important to remember that the modes come in orthogonal pairs, so the first ten modes can be thought of representing only five motions.

Full state feedback design in state space considers the control force $f$ to be of 
the form

$$
f=-G q
$$

where $G$ has as many rows as inputs. Substituting the result from equation (3.13) into equation (2.30) produces

$$
\dot{q}=(\bar{A}-\bar{B} G) q
$$

Physically this represents a controller that can measure every state in the model and then using these measurements, feed back force to the actuators. This model is not representative of the actual system because all of the states cannot be directly measured. The only states that can be directly measured are those measured by the piezos. The matrix $B$ represents the location of the inputs to the system.

At this point the poles of $(\bar{A}-\bar{B} G)$ can be chosen to be any value by the appropriate choice of the $G$ matrix. The limitation on the choice of the poles is that the more the poles are moved the greater the values in the $G$ matrix and thus the greater the required actuator force.

Consider an external disturbance expressed as

$$
\left[\begin{array}{c}
\dot{q}_{1} \\
\dot{q}_{2}
\end{array}\right]=\left[\begin{array}{cc}
0 & 1 \\
-\Lambda & -2 \zeta \sqrt{\Lambda}
\end{array}\right]\left[\begin{array}{c}
q_{1} \\
q_{2}
\end{array}\right]+\left[\begin{array}{c}
0 \\
\Psi^{T} B
\end{array}\right] f+\left[\begin{array}{c}
0 \\
\Psi^{T} F
\end{array}\right] f_{d}
$$

where $f_{d}$ is the external disturbance and $F$ is a pointer to show where the external disturbance acts. Now equation (3.15) can be written in compact form as

$$
\dot{q}=(\bar{A}-\bar{B} G) q+\bar{F} f_{d}
$$

Using this equation the closed loop response of the system to an external disturbance can be determined. 


\subsubsection{Observer-Based Feedback Design}

It is not practical to measure all the states of a model, as noted in the discussion of full state feedback. A more feasible approach to control design is to use an observer. An observer estimates the states of the system by measuring only a subset of the states, through the sensors in the system [9]. The estimated states, $\hat{q}$, are then used to implement feedback in the same manner as full state feedback. The key difference is that the feedback is based on the estimated states, $\hat{q}$, not the actual states, $q$. Thus the control law is

$$
f=-G \hat{q}
$$

The observer is represented by the equation

$$
\dot{\hat{q}}=\bar{A} \hat{q}+\bar{B} f+K\left(y_{\text {sens }}-\bar{C}_{\text {sens }} \hat{q}\right)
$$

where $K$ is a matrix of gains and $y_{\text {sens }}$ is the sensor output represented by

$$
y_{\text {sens }}=\bar{C}_{\text {sens }} q
$$

Under the assumption that the sensor output, $y_{\text {sens }}$ represents both position and velocity, $\bar{C}_{\text {sens }}$ can be expressed as

$$
\bar{C}_{\text {sens }}=\left[C_{\text {sens }} \Psi C_{\text {sens }} \Psi\right]
$$

Consider the example of a four node system with rotations in $x, y$, and $z$ at each node. The first two nodes represent a piezo on the top of the torus and the second two nodes represent a piezo on the bottom of the torus. The angle $\theta$ is the angle from the $x$ axis, in the $x y$-plane to the center of the piezo. Reference Figure 2.1 for a definition of the coordinate system. 
In this case there are two outputs making the vector $y_{\text {sens }}$ a $2 x 1$. The matrix $C_{\text {sens }}$ is then defined as

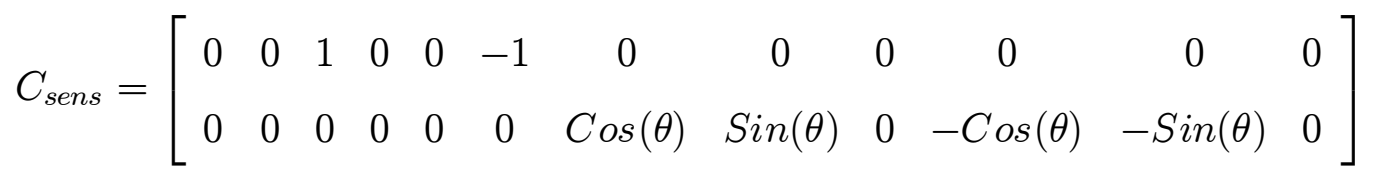

where the states are

$$
\left[\begin{array}{l}
\operatorname{rot} x 1 \\
\operatorname{roty} 1 \\
\operatorname{rot} z 1 \\
\operatorname{rot} x 2 \\
\operatorname{roty} 2 \\
\operatorname{rot} z 2 \\
\operatorname{rotx} 3 \\
\operatorname{roty} 3 \\
\operatorname{rot} z 3 \\
\operatorname{rot} x 4 \\
\operatorname{roty} 4 \\
\operatorname{rot} z 4
\end{array}\right]
$$

It is clear from equation (3.18) that the observer is a dynamic system. Thus the estimated states have a dynamic response. The error between the estimated states and the actual states is expressed by

$$
\dot{e}=\left(\bar{A}-K \bar{C}_{\text {sens }}\right) e
$$

The speed at which the error converges to zero depends on the location of the poles of the matrix $\bar{A}-K \bar{C}_{\text {sens }}$. These poles can be chosen to be any value through 
the gain matrix $K$ as long as the system is observable. Observability is the parallel to controllability discussed above and is determined by finding the rank of the matrix

$$
\left[\begin{array}{lllll}
\bar{C}_{\text {sens }}^{T} & \bar{A}^{T} \bar{C}_{\text {sens }}^{T} & \left(\bar{A}^{T}\right)^{2} \bar{C}_{\text {sens }}^{T} & \cdots & \left(\bar{A}^{T}\right)^{k-1} \bar{C}_{\text {sens }}^{T}
\end{array}\right]
$$

If the rank is $k$, the number of states, then the system is observable [9].

The gain matrix, $K$, determines the poles of the observer and thus the dynamic response of the observer. It is generally desirable to have the observer respond much faster than the physical system. This is analogous to picking the gain matrix $G$ when designing a full state compensator, however there is one significant difference. Choosing large gains $G$ results in large control forces. The gains $K$ are internal to the system and do not manifest themselves in any physical actuation in the observer system so there is no direct limitation on their value. It is, however, desirable to limit the gains $K$ because they affect the magnitude of the compensator. A compensator with large magnitudes will have more sensitivity to noise.

The complete dynamic system, the physical system and the observer expressed by equations (2.30) and (3.18), can now be combined to produce

$$
\left[\begin{array}{c}
\dot{q} \\
\dot{\hat{q}}
\end{array}\right]=\left[\begin{array}{cc}
\bar{A} & -\bar{B} G \\
K \bar{C}_{\text {sens }} & \bar{A}-K \bar{C}_{\text {sens }}-\bar{B} G
\end{array}\right]\left[\begin{array}{l}
q \\
\hat{q}
\end{array}\right]+\left[\begin{array}{c}
\bar{F} \\
0
\end{array}\right] f_{d}
$$

The poles of this system can be shown to be equal to the poles of $\bar{A}-\bar{B} G$ and $\bar{A}-K \bar{C}[9]$. This means that the poles of the complete dynamic system are the combination of the poles that were chosen for full state feedback and the poles that were chosen for the observer. Using this information the gain matrices $G$ and $K$ can be chosen to fully define the response of the system.

The disadvantage of using an observer is that it adds additional dynamics to the system. This can lead to the problem of observation spillover. Observation spillover can produce instability in the system. Observers can be designed to roll off at higher frequencies in order to reduce the potential for instability. The spillover can 
be reduced if the sensor signals are filtered to remove contributions of higher order modes [19].

\subsubsection{Observation Spillover}

To determine the effect of the control forces on the uncontrolled modes one can examine the matrix equation

$$
\begin{array}{r}
{\left[\begin{array}{c}
\dot{q} \\
\dot{q}_{R} \\
\dot{e}
\end{array}\right]=\left[\begin{array}{ccc}
\bar{A}-\bar{B} G & 0 & -\bar{B} G \\
-\bar{B}_{R} G & \bar{A}_{R} & -\bar{B}_{R} G \\
0 & K \bar{C}_{R} & \bar{A}-K \bar{C}
\end{array}\right]\left[\begin{array}{c}
q \\
q_{R} \\
e
\end{array}\right]+\left[\begin{array}{c}
\bar{F} \\
\bar{F}_{R} \\
0
\end{array}\right] f_{d}} \\
y=\left[\begin{array}{lll}
\bar{C} & \bar{C}_{R} & 0
\end{array}\right]
\end{array}
$$

where the subscript $R$ represents the residual modes, therefore

$$
\bar{A}_{R}=\left[\begin{array}{cc}
0 & 1 \\
-\Lambda_{R} & -2 \zeta \sqrt{\Lambda_{R}}
\end{array}\right] \quad \bar{F}_{R}=\left[\begin{array}{c}
0 \\
\Psi_{R}^{T} F
\end{array}\right] \quad \bar{C}_{R}=\left[\begin{array}{ll}
C \Psi_{R} & 0
\end{array}\right]
$$

where $\Psi_{R}$ represents the residual modes of the system. Development of these equations are found in many sources [19]. While $q_{R}$ is infinite dimensional, in reality only a finite number of modes can be excited in the system. The actual system can be approximated by choosing a finite dimension $q_{R}$ without significant loss of accuracy assuming that a large enough number of modes are considered [19].

The term $K \bar{C}_{R}$ in equation (3.26) demonstrates why observation spillover can cause instability. This presence of this term changes the eigenvalues of the system and has the potential to move them into the right hand side of the s-plane. The residual modes will not necessarily be moved into an unstable region of the s-plane but in the case of little or no damping they are close to imaginary axis and are the most vulnerable [19]. 


\subsubsection{Optimal Control}

The previous discussion of control design has considered using pole placement techniques. When multiple actuators are used the process of solving for the gain matrix becomes complicated because the gain matrix is not unique. One way to choose the gain matrix is by using optimal control. Optimal control centers around choosing a cost function to minimize. The cost function, or performance index, for the linear regulator problem can be formulated as

$$
J=\frac{1}{2} \int_{t_{0}}^{t_{f}}\left(x^{T} Q x+u^{T} R u\right) d t
$$

where $x$ represents the states and $u$ represents the control inputs. The matrices $Q$ and $R$ are symmetric positive definite weighting matrices. The larger $Q$ is, the more importance the control places on returning the system to zero. The larger $R$ is, the more importance the control places on minimizing the control force [11]. The optimal control law is

$$
u(t)=-R^{-1} B^{T} S(t) x(t)
$$

where

$$
Q-S(t) B R^{-1} B^{T} S(t)+A^{T} S(t)+S(t) A+\frac{d S(t)}{d t}=0
$$

under the condition that $S\left(t_{f}\right)=0$. The solution for $S(t)$ gives the optimal linear regulator control law, causing $J$ to be a minimum. For most problems of interest this problem must be solved numerically [11]. The commercial computer code MATLAB has built in functions to solve the optimal control problem.

\subsubsection{Velocity Feedback}

A velocity feedback control algorithm is a straightforward method to add damping to a system. It is a practical way to implement feedback control and does not require the 
use of an observer and the problems associated with observers. Unfortunately velocity feedback algorithms have problems as well. A velocity feedback controller does not roll off, so it applies gain even at high frequencies. Observer-based controllers can be designed to roll off at higher frequencies thereby limiting the potential for instability in the higher order modes.

Velocity feedback design considers the control force $f$ to be of the form

$$
f=-G y_{\text {sens }}=-G C_{\text {sens }} \dot{x}
$$

The most obvious differences between velocity feedback and full state modal feedback is that force is dependent not on the modal states, $q$, but the sensor outputs in real coordinates, $C_{\text {sens }} \dot{x}$. In this case the sensor output is the time rate of change of voltage from the piezos, represented by the equation

$$
y_{\text {sens }}=C_{\text {sens }} \dot{x}
$$

The time rate of change of the voltage is proportional to the difference between the rates of rotations at each end of the piezo.

Consider the example of a four node system with rotations in $x, y$, and $z$ at each node. The first two nodes represent a piezo on the top of the torus and the second two nodes represent a piezo on the bottom of the torus. The angle $\theta$ is the angle from the $x$ axis, in the $x y$-plane to the center of the piezo. See Figure 2.1 for a definition of the coordinate system.

In this case there are two outputs making the vector $y_{\text {sens }}$ a $2 \times 1$. The matrix $C_{\text {sens }}$ is then defined as

$$
C_{\text {sens }}=\left[\begin{array}{cccccccccccc}
0 & 0 & 1 & 0 & 0 & -1 & 0 & 0 & 0 & 0 & 0 & 0 \\
0 & 0 & 0 & 0 & 0 & 0 & \operatorname{Cos}(\theta) & \operatorname{Sin}(\theta) & 0 & -\operatorname{Cos}(\theta) & -\operatorname{Sin}(\theta) & 0
\end{array}\right]
$$


where the states are

$$
\left[\begin{array}{l}
\operatorname{rot} x 1 \\
\operatorname{rot} y 1 \\
\operatorname{rot} z 1 \\
\operatorname{rot} x 2 \\
\operatorname{roty} 2 \\
\operatorname{rot} z 2 \\
\operatorname{rot} x 3 \\
\operatorname{roty} 3 \\
\operatorname{rot} z 3 \\
\operatorname{rot} x 4 \\
\operatorname{rot} y 4 \\
\operatorname{rot} z 4
\end{array}\right]
$$

At this point the sensor output, $y_{\text {sens }}$ can be solved for this hypothetical case. This is proportional to the voltage output from the piezo. This "voltage" can now be multiplied by the gain matrix $G$ represented by

$$
\left[\begin{array}{cc}
g 1 & 0 \\
0 & g 2
\end{array}\right]
$$

where $g 1$ and $g 2$ represent the gains applied to each actuator. The output multiplied by the gains represents the control force applied to the tor us.

The full equation of motion can now be represented by

$$
\left[\begin{array}{c}
\dot{q}_{1} \\
\dot{q}_{2}
\end{array}\right]=\left[\begin{array}{cc}
0 & 1 \\
-\Lambda & -2 \zeta \sqrt{\Lambda}
\end{array}\right]\left[\begin{array}{l}
q_{1} \\
q_{2}
\end{array}\right]-\Psi^{T} B G C_{s e n s} \Psi q_{2}
$$

by remembering that $\dot{x}=\Psi q_{2}$ from equations (2.21) and (2.26).

The input force from each piezo then needs to be transferred back to the appropriate degrees of freedom through the $B$ matrix. Reconsidering the previous example, the $B$ matrix is defined as 


$$
B=\left[\begin{array}{cc}
1 & 0 \\
0 & 0 \\
0 & 0 \\
-1 & 0 \\
0 & 0 \\
0 & 0 \\
0 & \operatorname{Cos}(\theta) \\
0 & \operatorname{Sin}(\theta) \\
0 & 0 \\
0 & -\operatorname{Cos}(\theta) \\
0 & -\operatorname{Sin}(\theta) \\
0 & 0
\end{array}\right]
$$

Now the equation of motion can be represented as

$$
\left[\begin{array}{c}
\dot{q}_{1} \\
\dot{q}_{2}
\end{array}\right]=\left[\begin{array}{cc}
0 & 1 \\
-\Lambda & -2 \zeta \sqrt{\Lambda}-\Psi^{T} B G C_{\text {sens }} \Psi
\end{array}\right]\left[\begin{array}{l}
q_{1} \\
q_{2}
\end{array}\right]
$$

It can clearly be seen here that the feedback force adds only damping to the system by affecting only the $q_{2}$ states which represent $\dot{r}$, the modal velocities.

The response of the system to a disturbance can be modeled as

$$
\left[\begin{array}{c}
\dot{q}_{1} \\
\dot{q}_{2}
\end{array}\right]=\left[\begin{array}{cc}
0 & 1 \\
-\Lambda & -2 \zeta \sqrt{\Lambda}-\Psi^{T} B G C_{\text {sens }} \Psi
\end{array}\right]\left[\begin{array}{c}
q_{1} \\
q_{2}
\end{array}\right]+\left[\begin{array}{c}
0 \\
\Psi^{T} F
\end{array}\right] f_{d}
$$

where $f_{d}$ is a disturbance force and $F$ is a pointer indicating the degree(s) of freedom that the disturbance acts on.

The results section contains a discussion of the impulse response of this system. 


\subsection{Summary}

The force applied to the torus by the piezo actuators has been modeled using BernoulliEuler beam theory. Using this simplification, the piezo film measures the difference between the rotations at its ends. The piezo also produces two opposing moments at its ends when a voltage is applied to it.

Pole placement techniques are initially examined to attenuate vibration in the torus. Full state modal feedback is originally assumed to develop the equations. Using full state feedback the control force is assumed to depend on all of the states of the model. Next a modal observer is designed based on using the piezos as position and velocity sensors. Using the modal observer, the control force is based on the estimated states. The observer has dynamics which doubles the number of poles in the total system. The problem of observation spillover can make an observer-based controller go unstable. The use of optimal control is discussed as a preferred method for choosing the control gains.

Finally a simpler controller is designed using velocity output feedback. Velocity feedback is a simple method of controlling a structure but has the disadvantage that it does not roll off at high frequencies. Another disadvantage of velocity feedback is that the freedom to shape the closed loop response is much more limited.

These controllers are not chosen because they are the best for this application. They are chosen as a simple and effective demonstration of the control of an inflatable structure. 


\section{Chapter 4}

\section{Results}

\subsection{Introduction}

The aspect ratio and inflation pressure of an inflated torus has significant effect on its dynamics. Case studies of different aspect ratios, inflation pressures, and the effect of adding PVDF patches to the torus are examined in this section. Two control methods are examined, observer based state feedback and direct output velocity feedback. Optimal control is explored as a method for choosing the controller gains. The time response to an impulse excitation for both observer based state feedback and velocity feedback control methods are presented. The effect of observer spillover is examined for two different observer designs and the importance of considering observer spillover is illustrated.

\subsection{Effect of Torus Aspect Ratio on Mode Shapes}

The aspect ratio of the torus has a significant impact on not only the natural frequencies of the torus, but also on the mode shapes. The aspect ratio of a torus is defined by the radius of the tube divided by the radius of the ring, see Figure 1.2. The torus of interest here has a small aspect ratio so all of the lower modes are orthogonal pairs of ring modes, alternating in and out of the plane of the torus. Looking at the higher order modes of the torus, different types of modes are found. There are 
Table 4.1: Frequencies for Toroids of Different Aspect Ratios

\begin{tabular}{l|cccc}
\hline \hline & A. Ratio 0.04 & A. Ratio 0.08 & A. Ratio 0.16 & A. Ratio 0.32 \\
\hline Mode 1 & 3.40653 & 5.41651 & 8.6288 & 13.1871 \\
Mode 2 & 3.40653 & 5.41651 & 8.6288 & 13.1871 \\
Mode 3 & 5.00177 & 7.62025 & 11.24832 & 15.0978 \\
Mode 4 & 5.00177 & 7.62025 & 11.24832 & 15.0978 \\
Mode 5 & 7.6564 & 12.67014 & 20.02911 & 21.83219 \\
Mode 6 & 7.6564 & 12.67014 & 20.02911 & 25.85162 \\
Mode 7 & 8.76011 & 13.95878 & 20.92717 & 26.78526 \\
Mode 8 & 8.76011 & 13.95878 & 20.92717 & 26.78534 \\
Mode 9 & 13.00151 & 21.6558 & 28.04733 & 27.51872 \\
Mode 10 & 13.00151 & 21.65581 & 29.48152 & 27.51872 \\
Mode 11 & 13.75096 & 22.32614 & 32.4445 & 28.0326 \\
Mode 12 & 13.75096 & 22.32614 & 32.4445 & 28.03261 \\
Mode 13 & 19.4775 & 27.9986 & 32.57246 & 28.32397 \\
Mode 14 & 19.4775 & 31.91182 & 32.57248 & 28.32399 \\
\hline
\end{tabular}

sloshing modes, where the tube expands and contracts from side to side, breathing modes, where the torus expands and contracts uniformly, axisymmetric modes like those studied by Liepins [16], and many other types of motions. The important fact is this, as the torus aspect ratio increases, these types of modes start to show up in the lower order modes. In general, the sequence of the modes, especially the higher order modes, changes significantly as the aspect ratio increases. Table 4.1 compares the natural frequencies of four toroids with different aspect ratios, these toroids do not have PVDF patches. The ring diameter is held constant for each of the models, at $15.24 \mathrm{~m}$ (600 in). Each of the models is meshed with 60 elements around the ring diameter and 12 elements around the tube diameter. The first model, with an aspect ratio of 0.04, has the dimensions of interest here. The Appendix contains plots of the first fourteen modes of the toroids with aspect ratios of 0.04 and 0.32 .

The significant impact on mode shapes with changing aspect ratio is very important. For a torus with the aspect ratio of a car tire inner tube, there may be only a few ring modes present. This can have a significant impact on experimental work when trying to measure the mode shapes of a torus. This effect is also important when 
considering active control of the torus because mode shapes directly impact modal controllability and the placement of actuators and sensors. If the motion of the torus, within the frequency range of interest, is not solely ring modes, the actuators may need to be placed differently to create an effective controller.

\subsection{Dynamic Effect of Passive PVDF}

Williams studied the dynamic effect of passive PVDF patches on a tensioned membrane [24]. A passive PVDF patch refers to the dynamic effect of the PVDF considering only its mass and stiffness without electric excitation. He found that adding the PVDF lowered the natural frequencies of the tensioned membrane slightly but had no significant effect on the mode shapes. Essentially the PVDF patch added mass without significantly effecting the stiffness.

Adding PVDF patches to the torus has a similar effect, considering the torus The frequencies are lowered slightly but the mode shapes essentially remain the same through the first 14 modes, at least for thin flexible patches and toroids of the aspect ratio considered here. Figure 4.1 shows the effect of passive PVDF patches on the natural frequency of the torus. Two PVDF configurations were analyzed. The PVDF was added first to just the outer surface of the torus. The second configuration had PVDF patches on both the outer and the top surfaces of the torus, as defined in Figure 2.1.

The effect of the bonding process has not been considered in this model. The additional mass is likely insignificant but, depending on the bonding process used, the stiffness may be significantly increased. Significant increases in stiffness could have additional effects on the response of the torus. Careful choice of bonding techniques should minimize changes to the dynamics of the torus. 


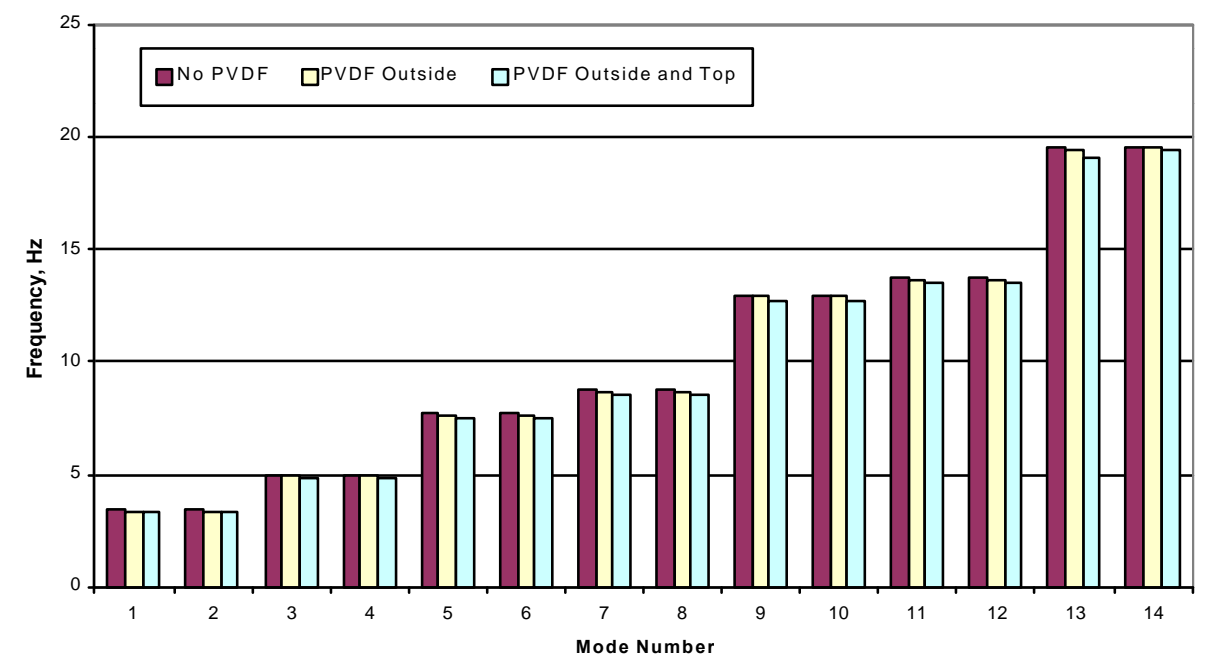

Figure 4.1: Effect of Passive PVDF Patches on Frequency.

\subsection{Effect of Pressure on Natural Frequencies}

Changing the pressure inside the torus has a significant effect on its natural frequencies. Figures 4.2 and 4.4 show the effect of changing pressure from 0.5 to 2.5 psi on the first fourteen modes of toroids with aspect ratios 0.04 and 0.32 respectively. The frequencies increase with increasing pressure, as expected. One less obvious, and more important, result is that for toroids of large aspect ratios, the order of the mode shapes changes significantly with increasing pressure. Charts showing types of modes at various inflation pressures for toroids with aspect ratios of 0.04 and 0.32 are shown in Figures 4.3 and 4.5 respectively. Again, the importance of the aspect ratio of the torus on its dynamic behavior is found to be very important. 


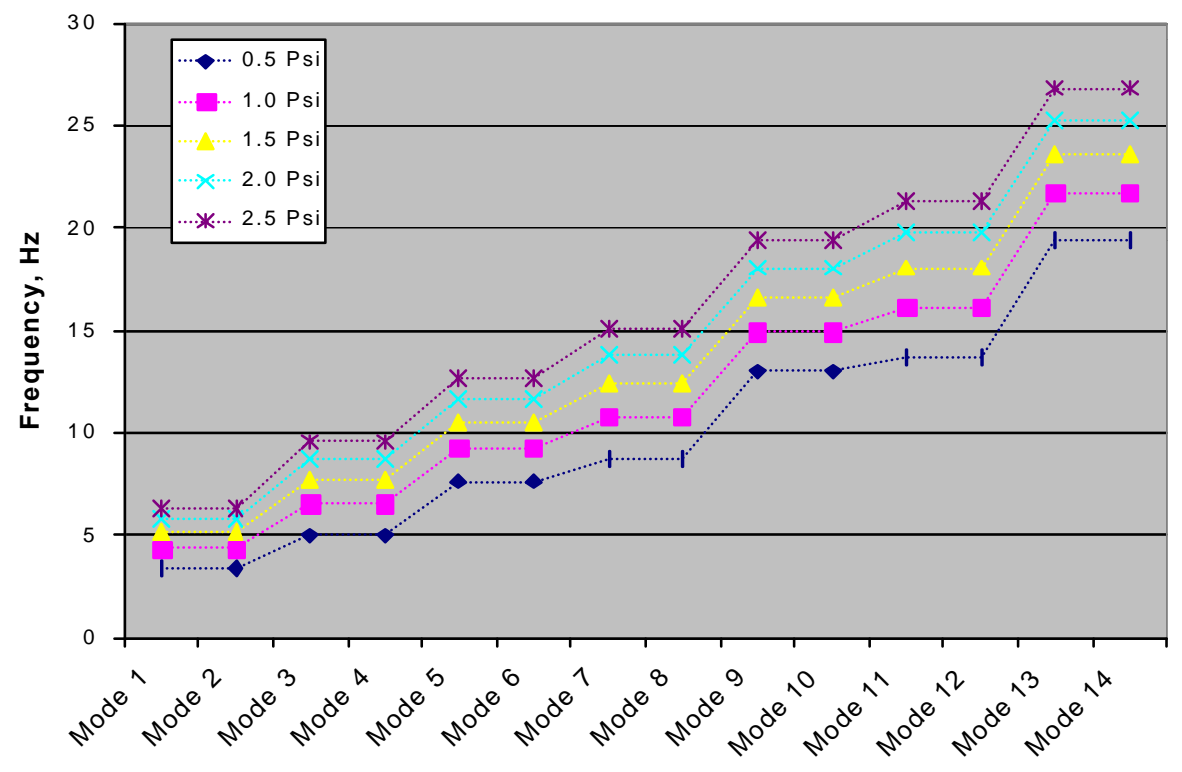

Figure 4.2: Effect of Pressure on Small Aspect Ratio (0.04) Torus Natural Frequencies

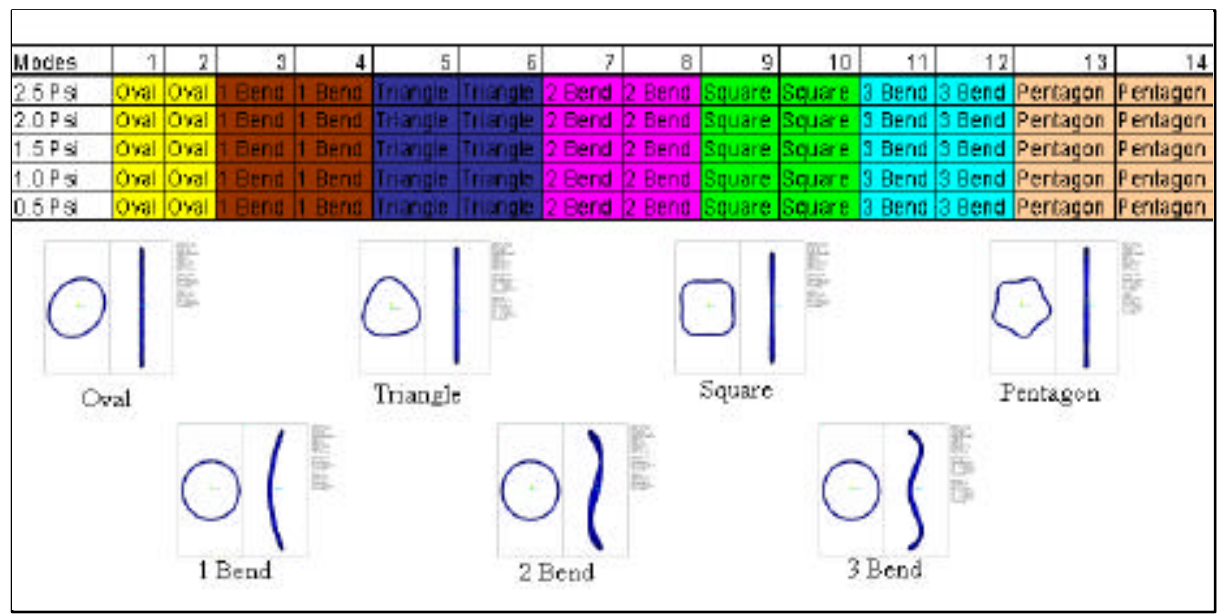

Figure 4.3: Effect of Pressure on Small Aspect Ratio (0.04) Torus Mode Shapes 


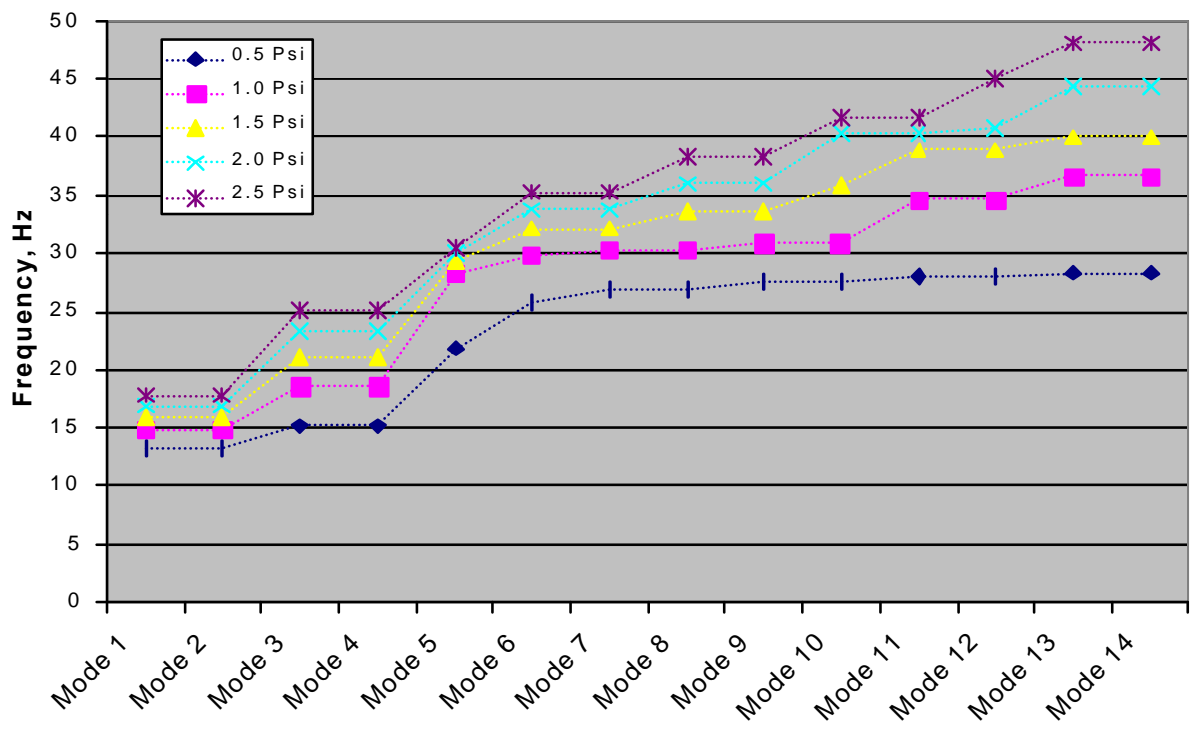

Figure 4.4: Effect of Pressure on Large Aspect Ratio (0.32) Torus Natural Frequencies

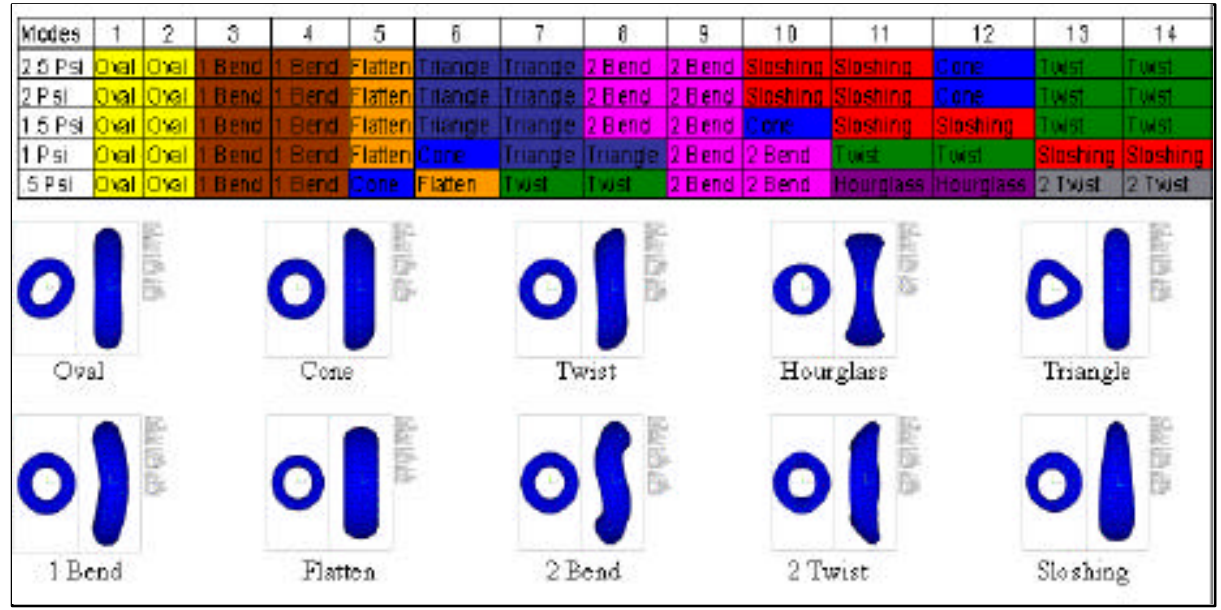

Figure 4.5: Effect of Pressure on Large Aspect Ratio (0.32) Torus Mode Shapes 


\subsection{Open Loop Response of Torus}

The open loop response of the torus to in-plane excitation, including the passive effect of the PVDF patches, is shown in Figures 4.6 and 4.7. Figures 4.8 and 4.9 show the open loop response to an out-of-plane excitation. The impulse applied in both the in-plane and out-of-plane cases is $4.4 \mathrm{e}-6 \mathrm{~N}^{*} \mathrm{~s}\left(1 \mathrm{e}-6 \mathrm{lbf}^{*} \mathrm{~s}\right)$. The damping seen in the response is due to the $1 \%$ modal damping in the model.

The response in the direction of excitation, either in-plane or out-of-plane, is clearly dominated by the mode shapes in that direction. The minimal response in the orthogonal direction is a summation of mode shapes in both directions as a result of the small amounts of coupling in the model due to slight asymmetries caused by the PVDF.

\subsection{Observer Based State Feedback Control}

The torus is controlled with twenty PVDF actuators, ten located on the outer surface and ten on the top surface of the torus, as shown in Figure 2.1. These actuators are modeled as applying opposing moments to the torus at their ends. The first ten modes of the structure are controlled and the response is simulated.

The gains, $g$, of the system were chosen using optimal control theory as discussed in Chapter Three. The objective of the design was to obtain the shortest settling time while not violating the maximum available actuator force. The poles of the observer were chosen using pole placement methods. The gains, $k$, for the observer were then calculated by MATLAB using the place command. The gains $k$ are not unique so MATLAB minimizes the norm of the matrix. These gains were found to

produce significant observer spillover, as discussed in the next section. New observer gains were then designed using optimal control theory. See the Appendix for details on the MATLAB codes used.

It is interesting to note the significant out-of-plane response to an in-plane excitation, and vice versa. This occurs due to the coupling induced by the gain 


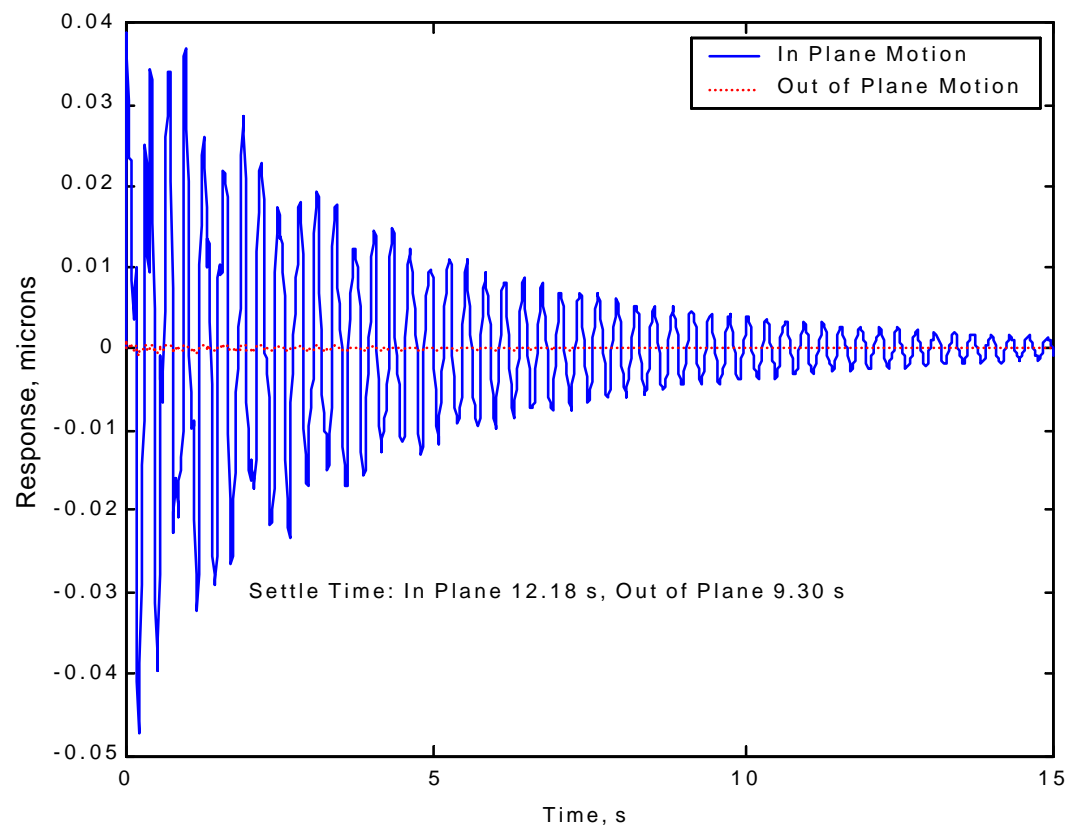

Figure 4.6: Open Loop Impulse Response to In-Plane Excitation.

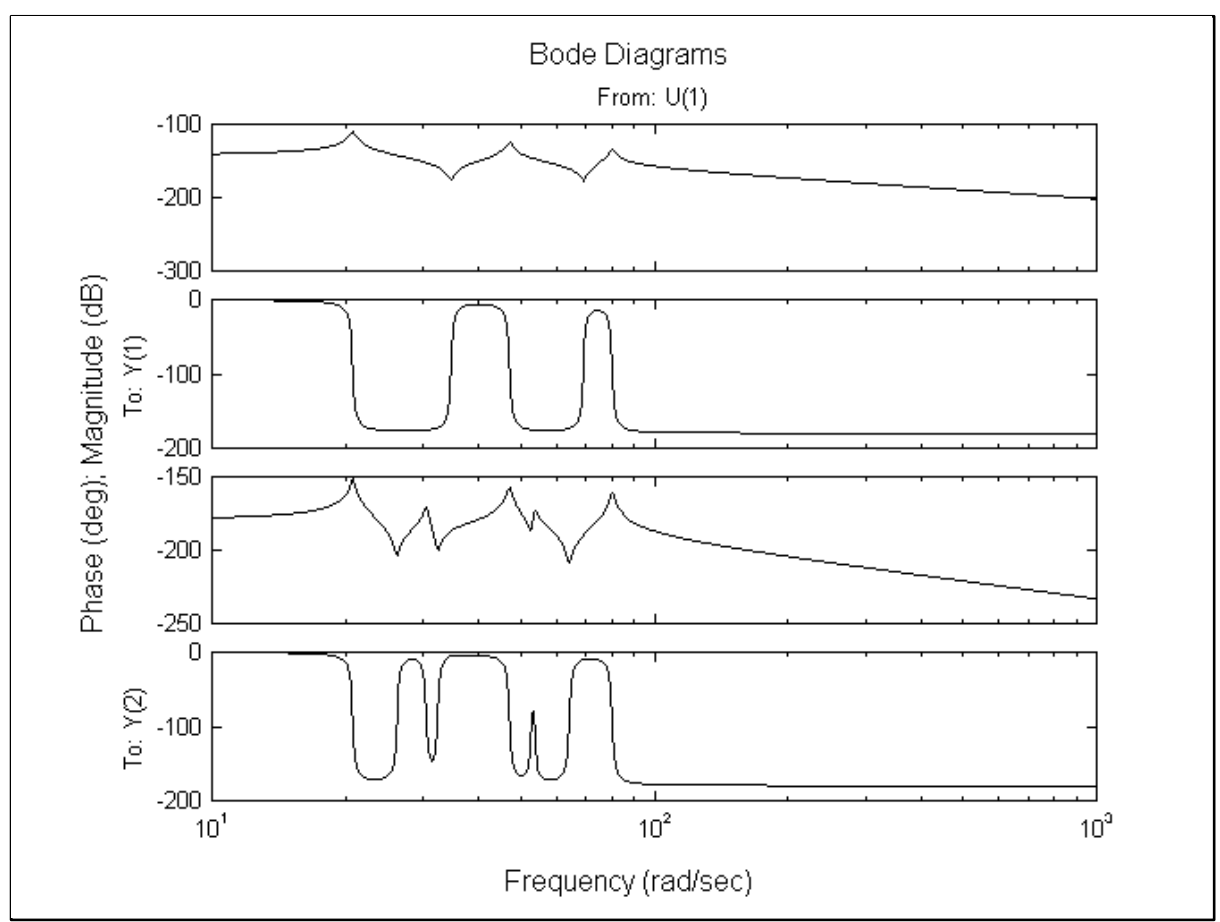

Figure 4.7: Open Loop Frequency Response to In-Plane Excitation. $\mathrm{Y}(1)=$ In-Plane-Response, $\mathrm{Y}(2)=$ Out-of-Plane Response 


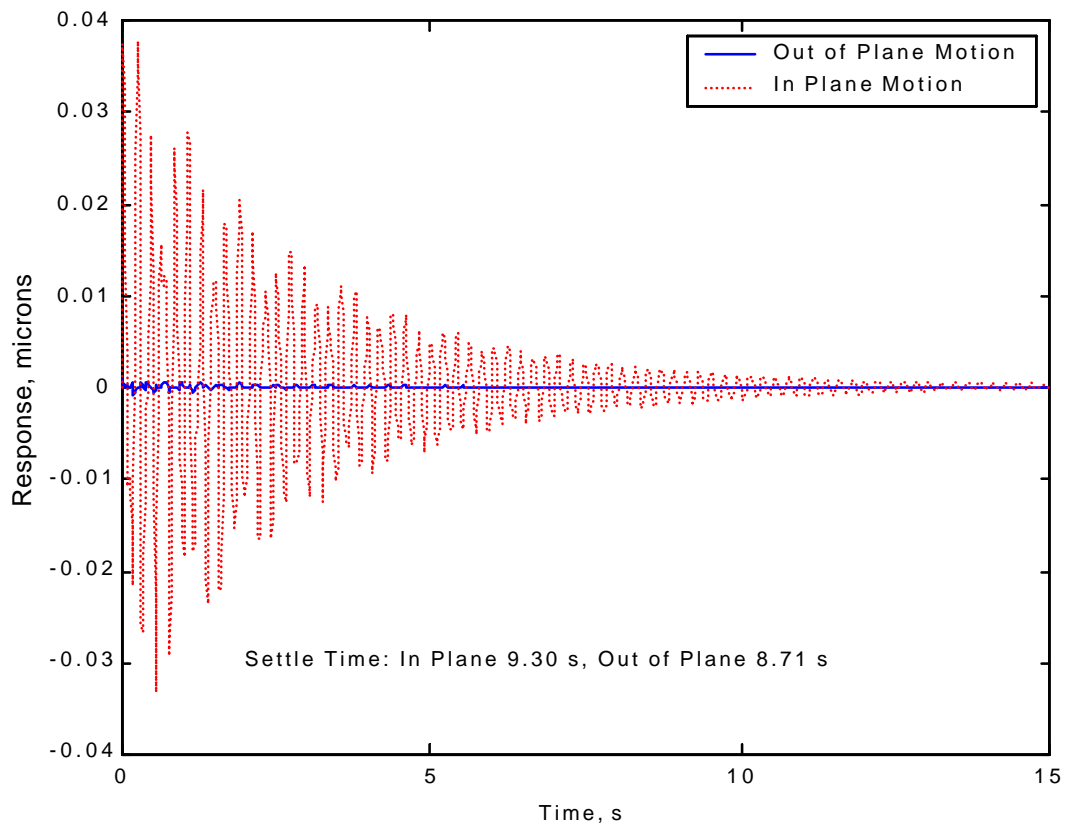

Figure 4.8: Open Loop Impulse Response to Out-of-Plane Excitation.

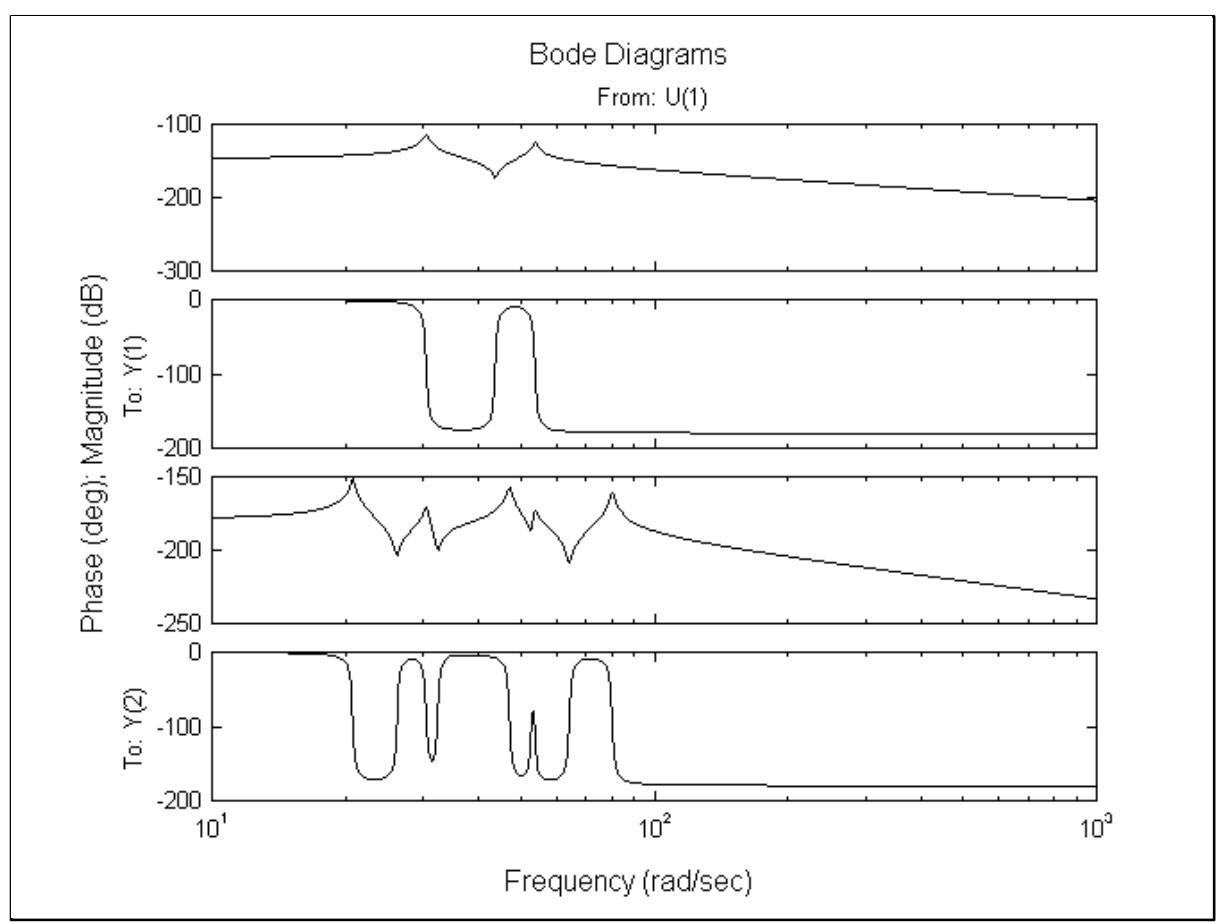

Figure 4.9: Open Loop Frequency Response to Out-of-Plane Excitation. $\mathrm{Y}(1)=$ Out-of-Plane Response, $\mathrm{Y}(2)=\mathrm{In}$-Plane Response 


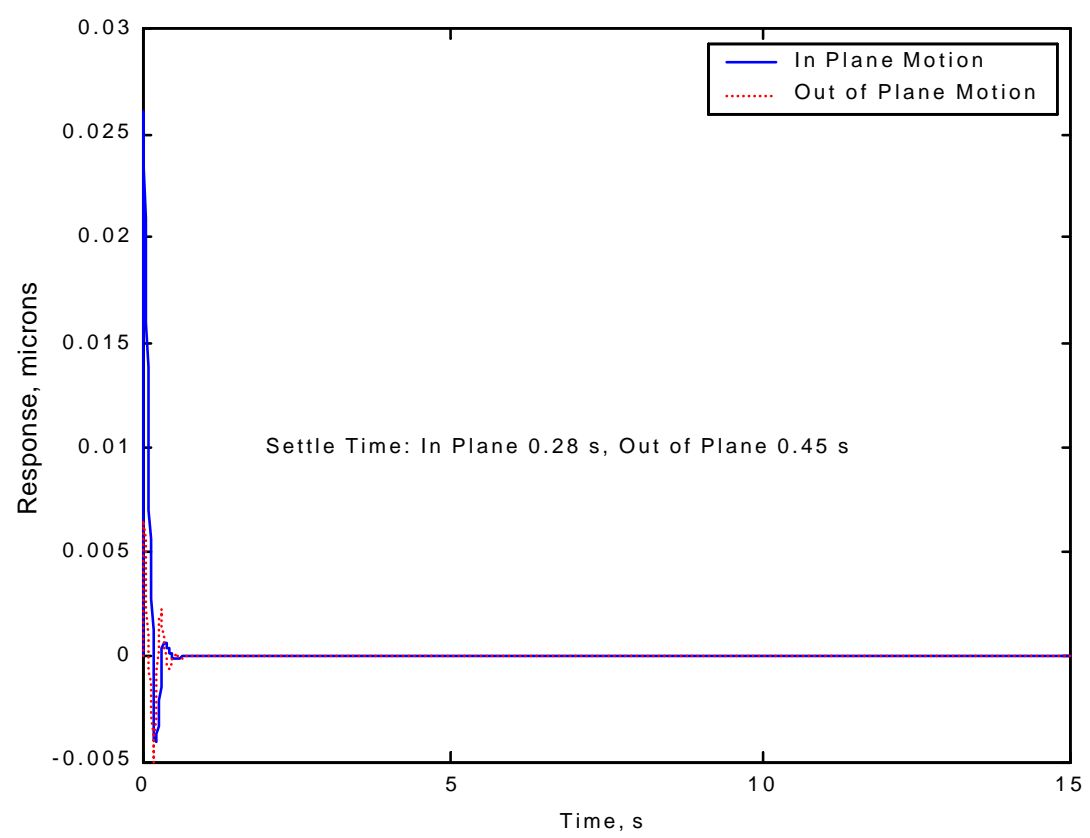

Figure 4.10: Observer Based Closed Loop Impulse Response to In-Plane Excitation, Observer Designed Using Pole Placement.
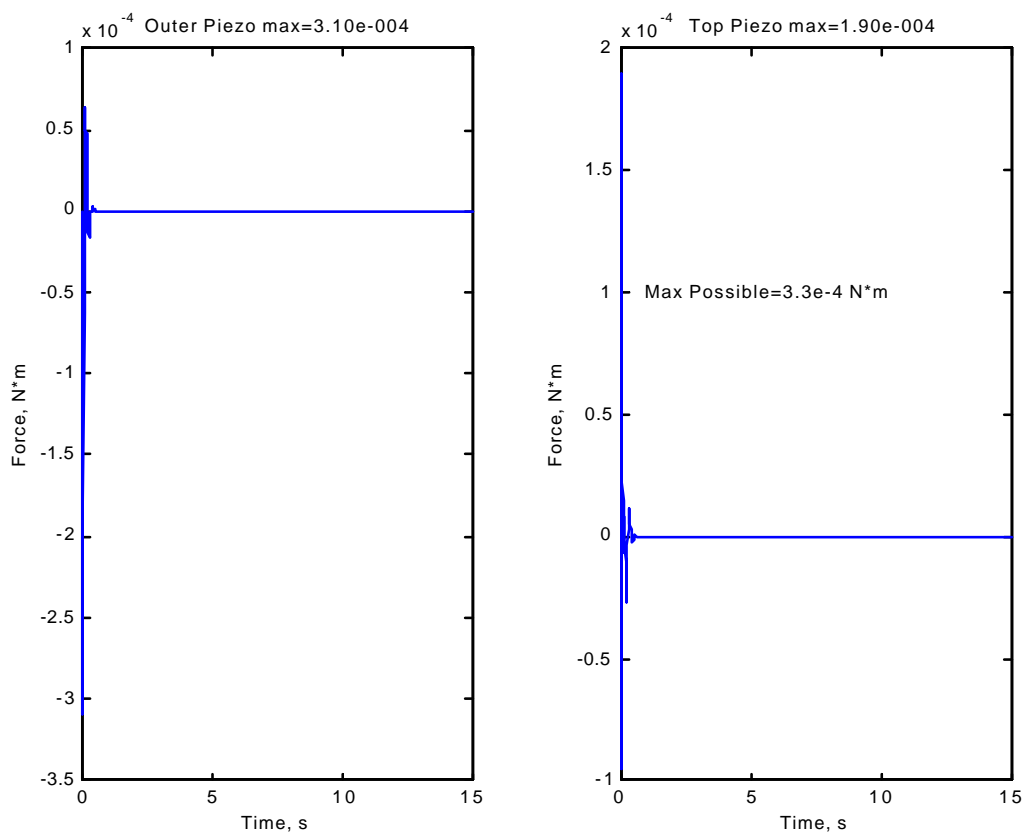

Figure 4.11: Observer Based Closed Loop Output Force for In-Plane Excitation, Observer Designed Using Pole Placement. 


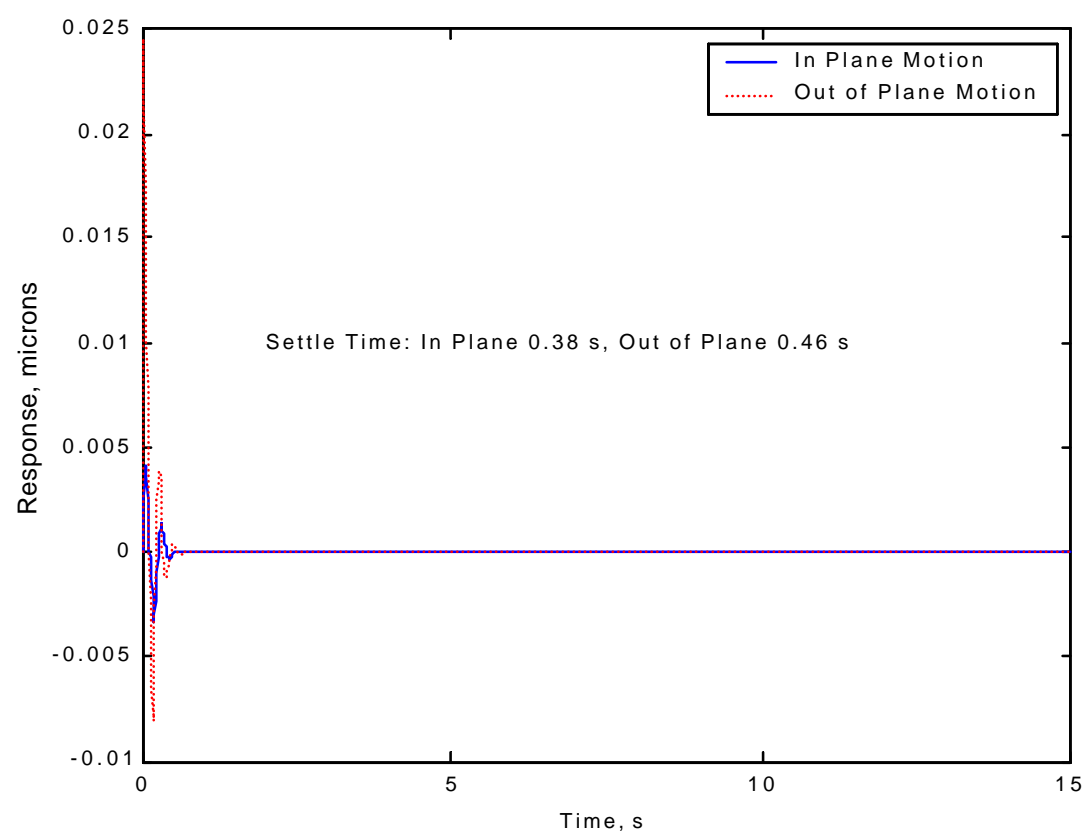

Figure 4.12: Observer Based Closed Loop Impulse Response to Out-ofPlane Excitation, Observer Designed Using Pole Placement.
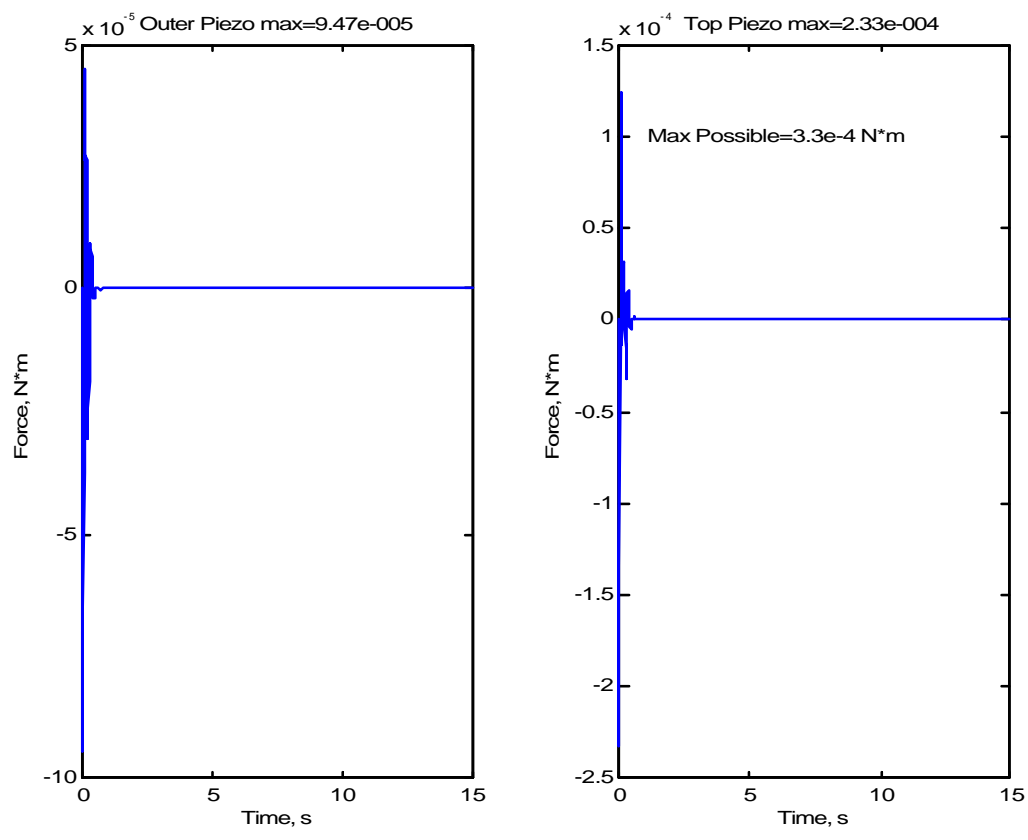

Figure 4.13: Observer Based Closed Loop Output Force for Out-of-Plane Excitation, Observer Designed Using Pole Placement. 


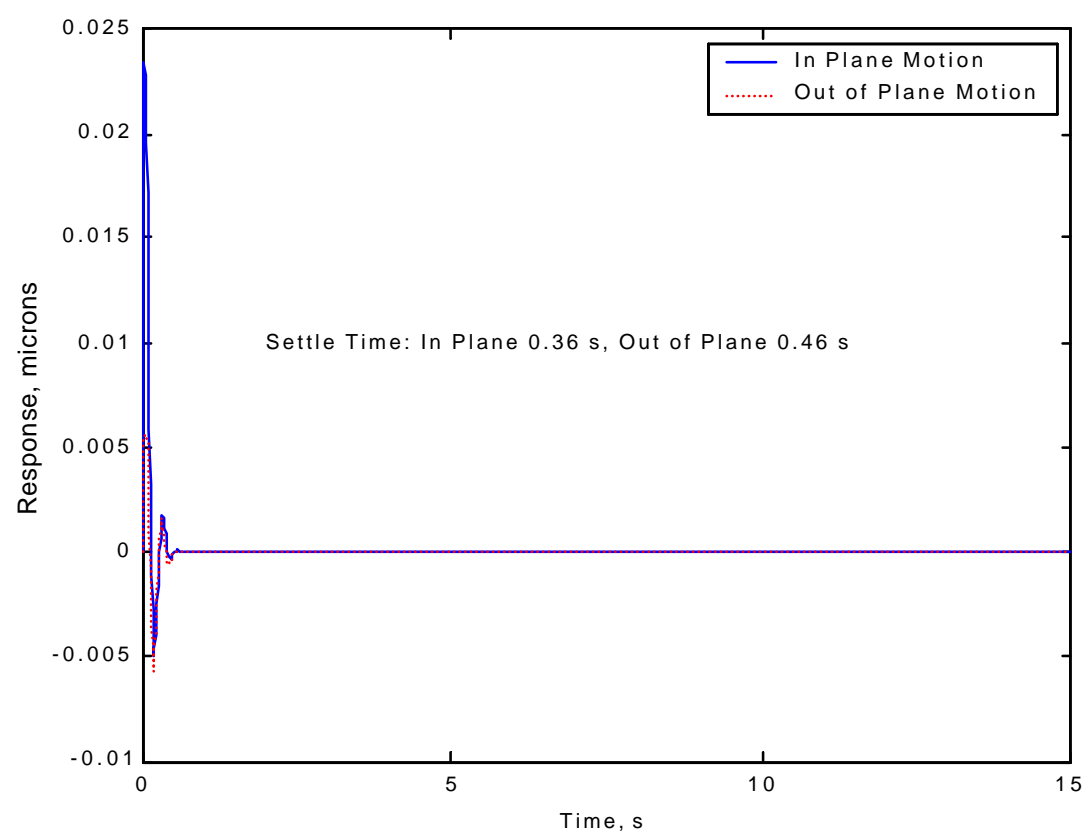

Figure 4.14: Observer Based Closed Loop Impulse Response to In-Plane Excitation, Observer Designed Using Optimal Control.
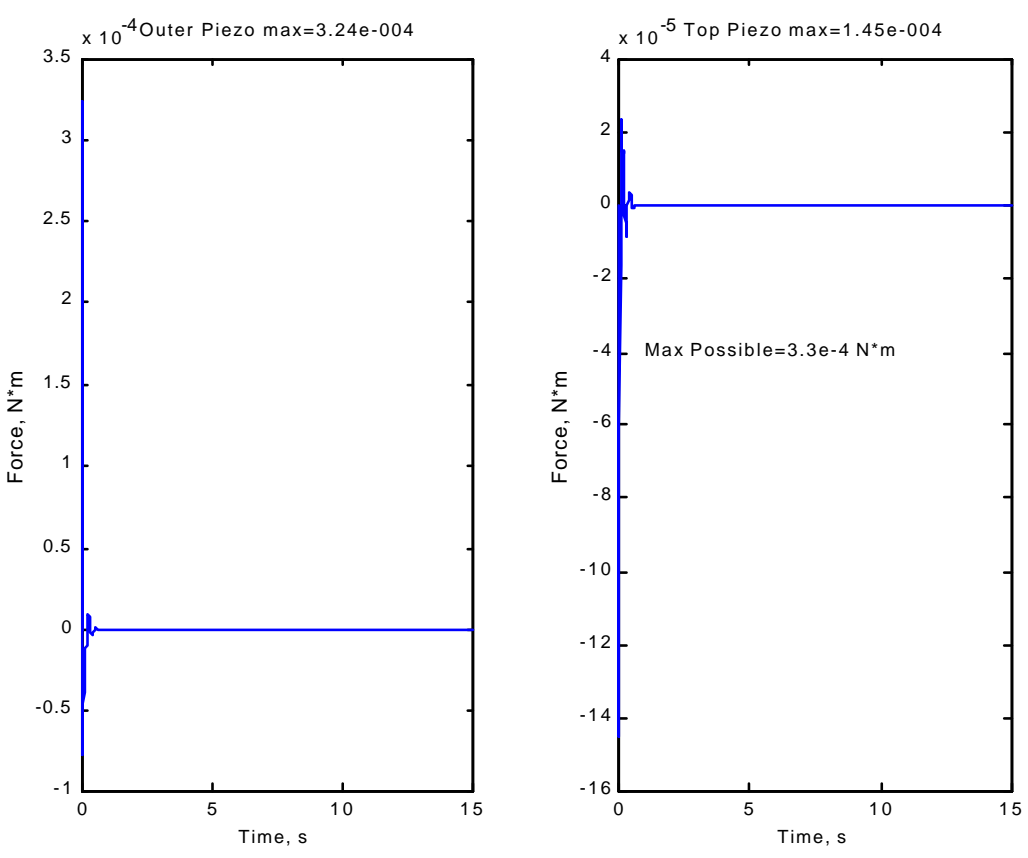

Figure 4.15: Observer Based Closed Loop Output Force for In-Plane Excitation, Observer Designed Using Optimal Control. 


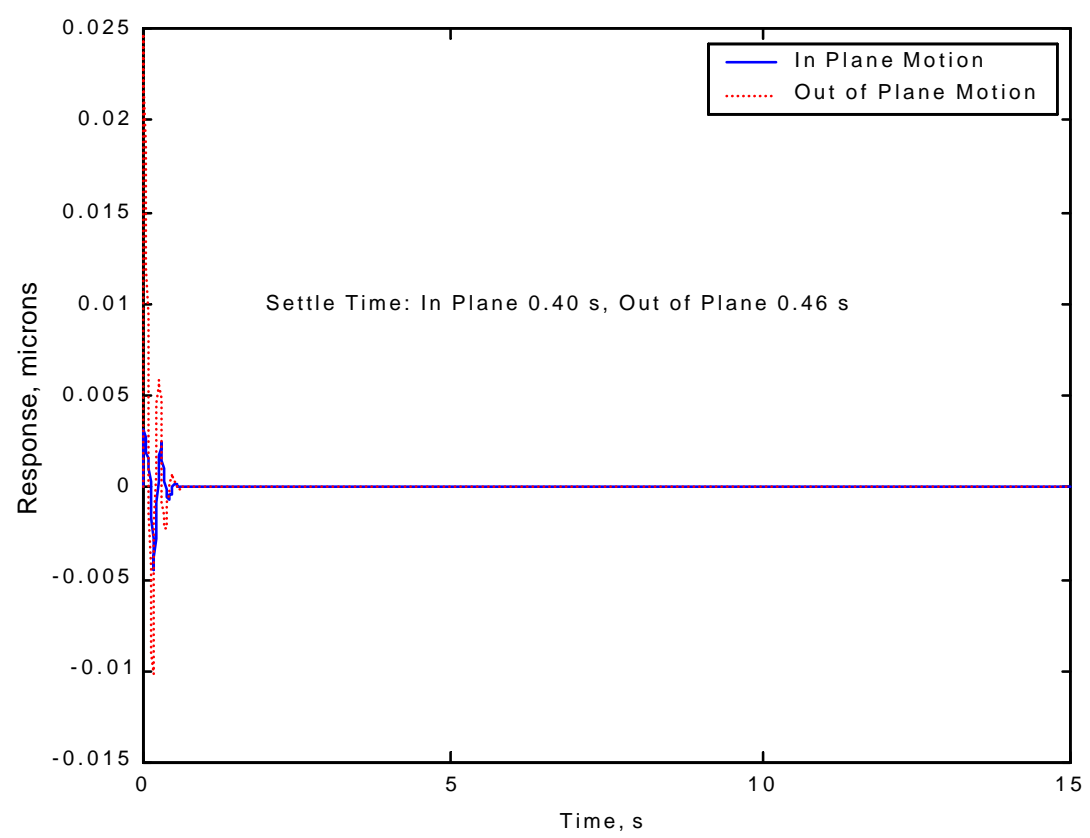

Figure 4.16: Observer Based Closed Loop Impulse Response to Out-ofPlane Excitation, Observer Designed Using Optimal Control.
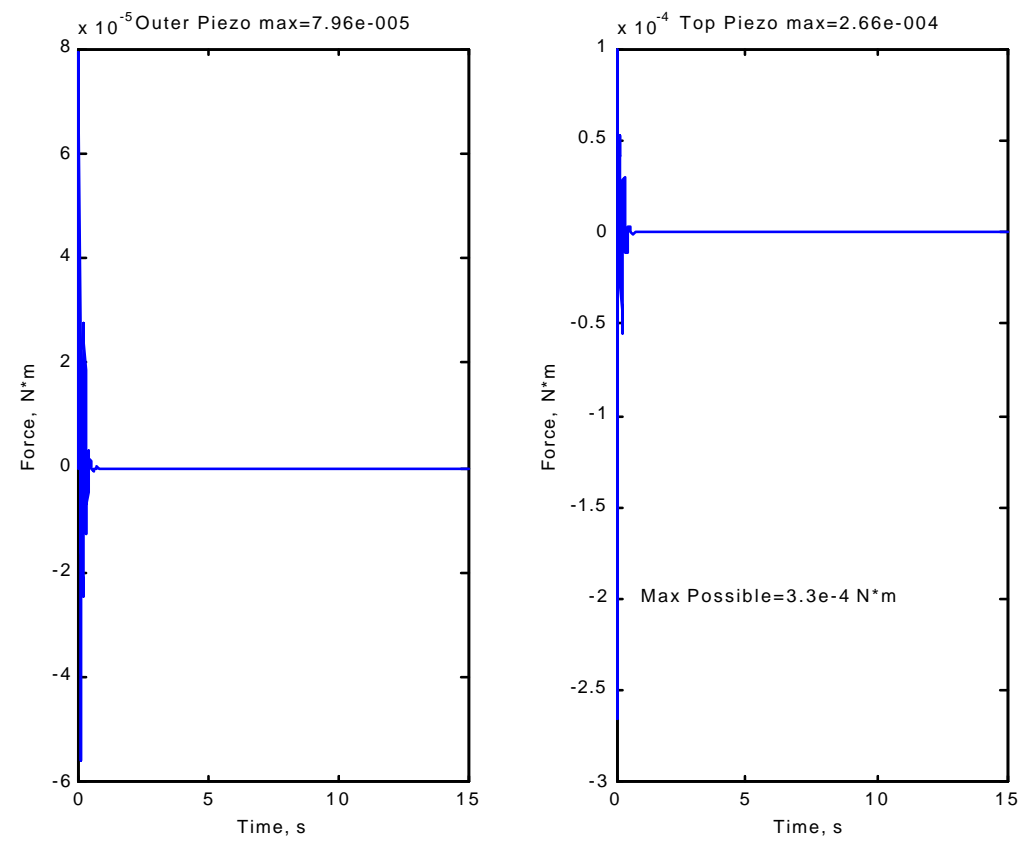

Figure 4.17: Observer Based Closed Loop Output Force for Out-of-Plane Excitation, Observer Designed Using Optimal Control. 
matrix. This is the problem of control spillover. Because the control response is a function of the response of each mode, the out-of-plane controller is excited by the in-plane response. This problem can be avoided by a more judicious choice of the gain matrix. The gain matrix can be chosen such that the in-plane response is only fed back to the in-plane actuators and the out-of-plane response to the out-ofplane actuators. While in theory this appears easy it is somewhat more difficult to implement.

\subsubsection{Effect of Observer Spillover}

Observation spillover can lead to instability in observers, as noted in Chapter Three. To examine this effect, as formulated in equation (3.26), twenty residual modes are considered for two observers designed using pole placement and optimal control. When considering the residual modes from the pole placement design, the system becomes unstable as can be seen from the time response in Figure 4.18 and noting

the fact that some of the eigenvalues of the system are in the right half of the s-plane. Using optimal control to design the observer, the system performance is degraded but it does not become unstable, as seen in Figure 4.19. It is important to note that the stability of the observer should not be concluded to be a function of the design method used. The purpose of this demonstration is to show that the effects of observer spillover need to be accounted for, the importance of which should be shown here. Generally speaking a compensator can be designed that rolls off at frequencies above the controlled frequencies, thereby reducing the problem of observation spillover. 


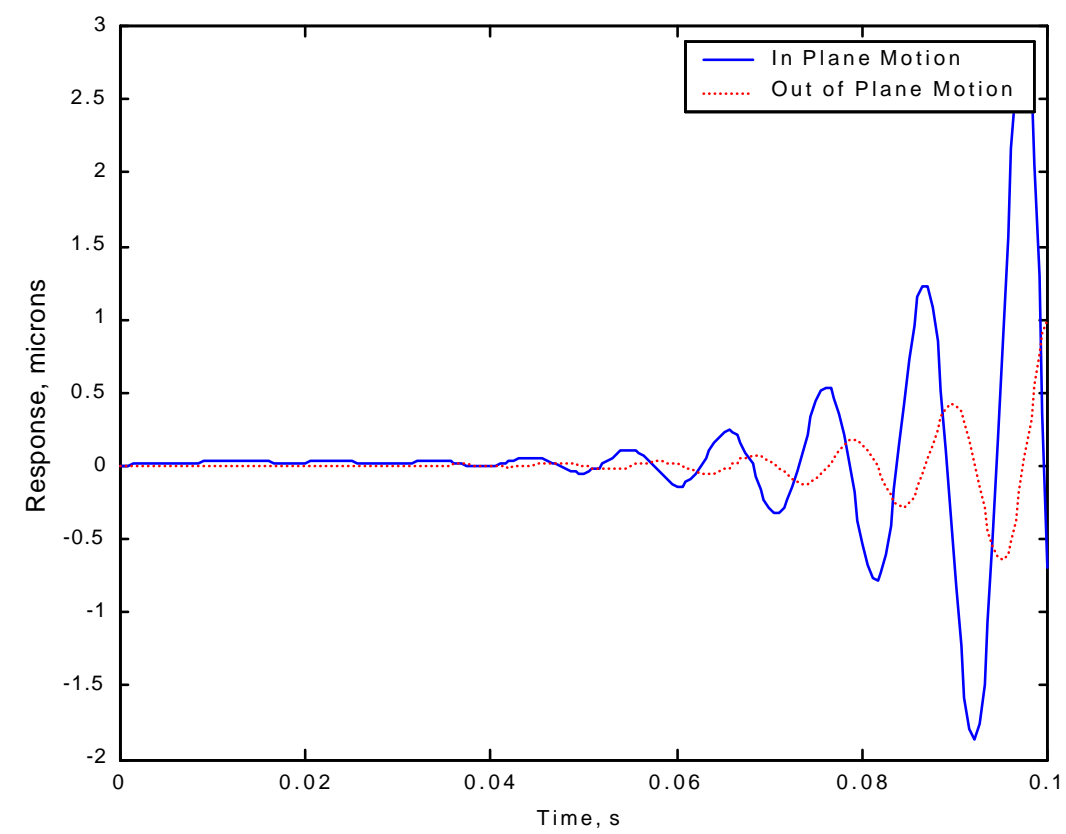

Figure 4.18: Impulse Response Considering Residual Modes using Pole Placement Techniques.

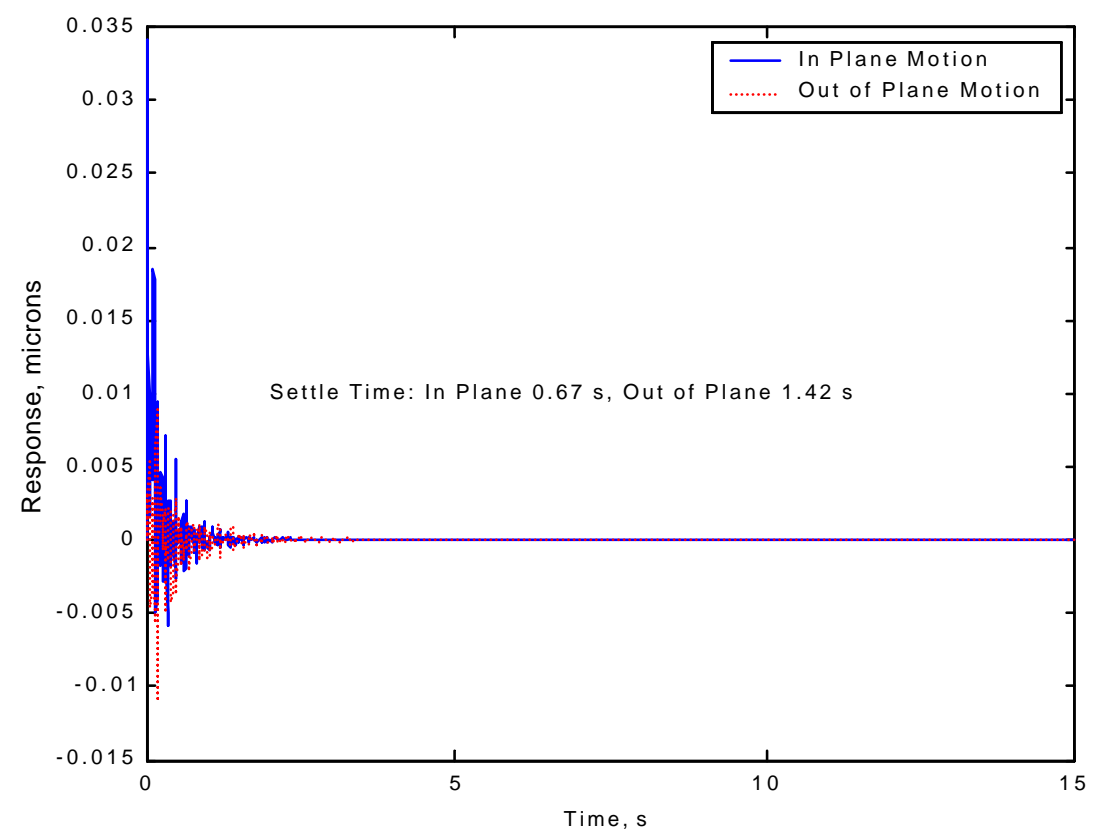

Figure 4.19: Impulse Response Considering Residual Modes using Optimal Control. 


\subsection{Results of Velocity Feedback Control}

The torus is controlled with twenty PVDF actuators, ten located on the outer surface and ten on the top surface of the torus, as shown in Figure 2.1. These actuators are modeled as applying opposing moments to the torus at their ends. The first ten modes of the structure are controlled and their response is simulated. The effect of the velocity feedback controller on the higher order modes is not considered here. Velocity feedback does not roll off at high frequencies, so the influence of the higher order modes can be significant.

The velocity feedback controller was able to reduce the impulse response settling time to about 1 second, based on a $5 \%$ of $\max$ value settling criteria. The impulse applied was $4.4 \mathrm{e}-6 \mathrm{~N}^{*} \mathrm{~s}\left(1 \mathrm{e}-6 \mathrm{lbf}^{*} \mathrm{~s}\right)$. The in-plane and out-of-plane impulse responses are shown in Figures 4.20 and 4.22. The maximum actuator force, for both the top and outer actuators is shown for each case in Figures 4.21 and 4.23. 


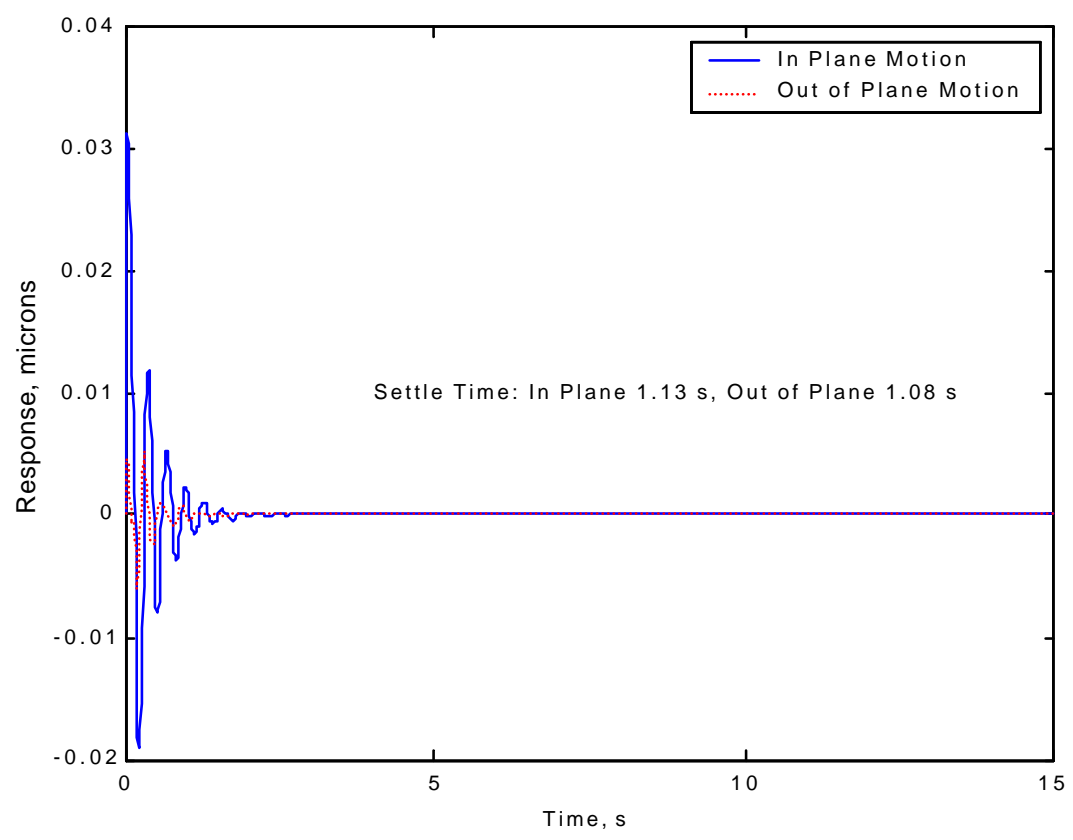

Figure 4.20: Velocity Feedback Closed Loop Impulse Response to In-Plane Excitation.
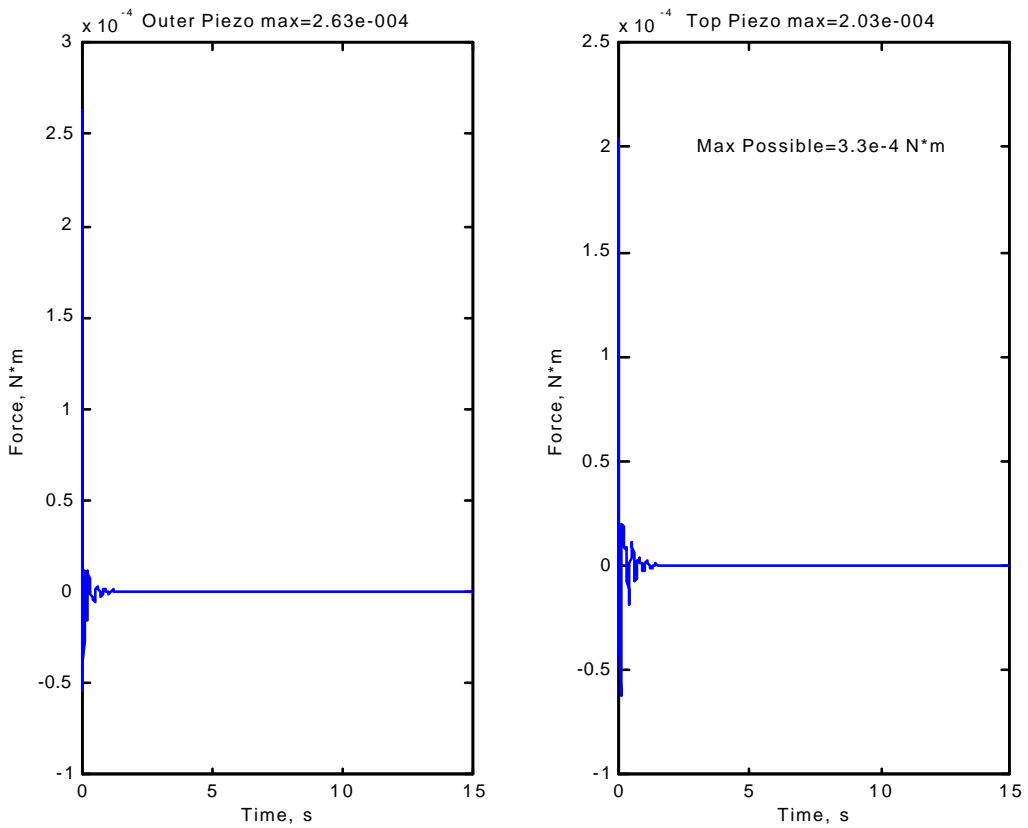

Figure 4.21: Velocity Feedback Closed Loop Output Force for In-Plane Excitation. 


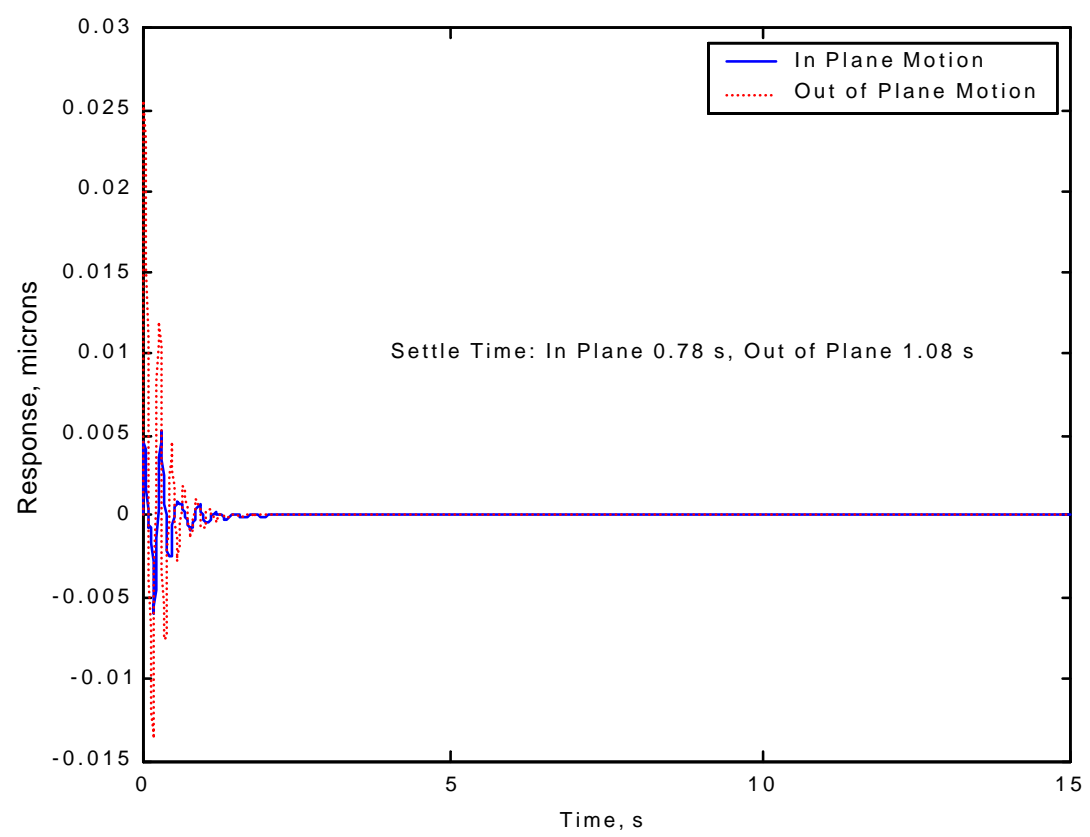

Figure 4.22: Velocity Feedback Closed Loop Impulse Response to Out-ofPlane Excitation.
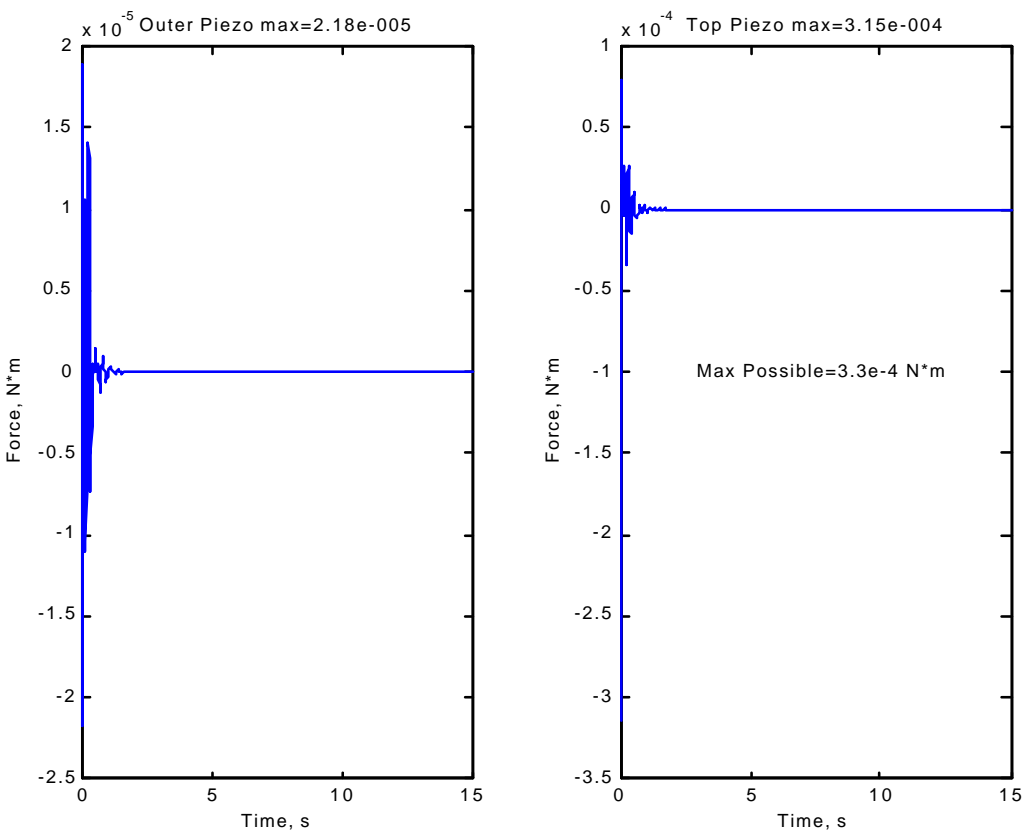

Figure 4.23: Velocity Feedback Closed Loop Output Force for Out-of-Plane Excitation. 


\subsection{Summary}

The aspect ratio and inflation pressure of an inflated torus has significant effect on its dynamics. Toroids of large aspect ratios experience greater changes in mode shapes with changing pressure. The addition of PVDF patches does not have a significant effect on the dynamic response of the torus, however the effect of the bonding process has not been considered. The results of implementing observer based state feedback and velocity feedback control methods are also presented in this section. Although spillover is only discussed for observers, it is also important for direct output feedback, because direct output feedback does not roll off at high frequencies. The controllers designed here verify that the vibratory response of the torus can be controlled using PVDF actuators.

Table 4.2: Controller Results

\begin{tabular}{|c|c|c|c|c|}
\hline & \multicolumn{3}{|c|}{ In-Plane Excitation } \\
\hline & \multicolumn{2}{|c|}{ In-Plane } & \multicolumn{2}{c|}{ Out-of-Plane } \\
\hline Controller & Response,s & Force,N*m & Response,s & Force,N*m \\
\hline Open Loop & 12.18 & - & 9.30 & - \\
Observer & 0.28 & $3.10 \mathrm{e}-4$ & 0.45 & $1.90 \mathrm{e}-4$ \\
Optimal Observer & 0.36 & $3.24 \mathrm{e}-4$ & 0.46 & $1.45 \mathrm{e}-4$ \\
Optimal w/Residual & 0.67 & & 1.42 & \\
Velocity Feedback & 1.13 & $2.63 \mathrm{e}-4$ & 1.08 & $2.03 \mathrm{e}-4$ \\
\hline
\end{tabular}




\section{Chapter 5}

\section{Conclusions}

\section{$5.1 \quad$ Introduction}

This thesis has investigated the feasibility of using PVDF to attenuate vibration in an inflated torus. To this end, the dynamics of the free-free torus have been investigated. The passive effect of adding PVDF to the structure has been examined. Finally a state space model has been created to implement standard control techniques to demonstrate the controllability of the structure using PVDF actuation.

\section{$5.2 \quad$ Key Results}

The inflation pressure and aspect ratio of a torus are found to have significant impact on its dynamic response. Increasing the inflation pressure generally increases the natural frequencies of the torus. This result should be expected since increasing inflation pressure essentially stiffens the torus. The vibratory response of toroids with small aspect ratios, skinny tubes, is dominated by ring modes. When looking at toroids with larger aspect ratios, toroids with fat tubes, the vibratory response contains many different types of modes. For this reason it may be more difficult to attenuate vibration in toroids with large aspect ratios. Also, increasing pressure

in toroids with large aspect ratios tends to affect the sequence of the modes. Any structural-acoustic interaction that could occur is not considered. 
Piezoelectric PVDF patches have a very limited effect on the vibratory response of toroids with small aspect ratios, considering only their passive effect. Generally the natural frequencies are decreased slightly, as should be expected by adding mass to the system, but there is little influence on the mode shapes. Only part of the stiffness of the PVDF patch is considered because the PVDF and Kapton are assumed to lie in the same plane instead of being layered.

Using a closed loop feedback system the vibratory response of an inflated torus can be attenuated significantly compared to its open loop response. The PVDF is modeled very simply. This simple model can be questioned in two ways. Is it appropriate to model the force transferred from the PVDF to the Kapton as two opposing moments? Is the calculation of the maximum possible amount of force generated by the PVDF correct? Vibratory control of an inflated torus is shown to be possible. The effect of spillover is significant when designing any vibration suppression system and must be considered in order to produce a viable design.

\subsection{Future Work}

The torus model here was validated by analysis performed by Liepins in 1965. Liepins analyzed and validated his models of inflated toroids, but with significantly greater stiffness. Test validation for very thin inflated shells is yet to be performed in detail.

The interaction between the PVDF and the host structure, the inflated torus, was treated as a beam. This is a great simplification of the structure. Much more detailed analysis of the interaction between the actuator and the torus needs to be performed to truly determine the potential effectiveness of vibration control of the structure.

The dynamics of the torus are treated as being linear with small deformations. These assumptions need to be investigated in detail to determine their validity. The materials used in inflated space structures often have significant nonlinear properties. There is also an important question as to whether or not vibration of thin inflated structures can really be treated as small deformation problems. This author believes 
that they can because the motion seen here is essentially motion of the entire tube, not local deformation of the tube wall, however at present this question has not been satisfactorily answered.

The control system designed here was not intended to produce an optimal response. The purpose of this control design was to demonstrate control of the torus. Practical implementation of control of this system demands a much more rigorous control design considering robustness and, as shown here, spillover.

This work has considered only structures that rely mainly on inflation pressure for their structural stiffness. Rigidization techniques are likely to be more popular for production systems because they do not rely on an internal pressure to maintain their stiffness. The ability of piezoelectric devices to control rigidizable structures needs to be examined. 


\section{Bibliography}

[1] Agnes, Gregory S. and Rogers, James W., Piezoelectric Excitation of Inflatable Space Structures for Modal Testing. SPIE Smart Materials and Structures Conference, March 2000.

[2] Cassapakis, C. and Mitch, T., Inflatable Structures Technology Development Overview. Paper 95-3738, AIAA, 1995.

[3] Chaudhry, Z. and Rogers, C.A., The Pin-Force Model Revisited. Journal of Intelligent Material Systems and Structures, Vol. 5, May 1994, pp 347-354.

[4] Derbes, B., Case Studies in Inflatable Rigidizable Structural Concepts for Space Power. Paper 99-1089, AIAA, 1999.

[5] Dosch, J.J., Inman Daniel J., and Garcia E., A Self-Sensing Actuator for Collocated Control. Journal of Intelligent Material Systems and Structures, Vol. 3, No. 1, Jan 1992, pp 166-185.

[6] Freeland, R. E., Significance of the Inflatable Antenna Experiment Technology. Paper 98-2104, AIAA, 1998.

[7] Freeland, R.E., Bilyeu, G.D., and Veal, G.R., Development of Flight Hardware for a Large, Inflatable-Deployable Antenna Experiment, International Astronautical Federation, Paper 95-1501, 1995.

[8] Freeland, R.E. and Bilyeu, G.D., In-Step Inflatable Antenna Experiment, International Austronautical Federation, Paper 92-0301, 1992. 
[9] Friedland, Bernard, Control System Design, An Introduction to State-Space Methods, McGraw Hill, Boston Massachusetts, 1986.

[10] Griffith, D. Todd and Main, John A., Modal Testing of an Inflated Thin Film Polyimide Torus Structure Proceedings, XVIII International Modal Analysis Conference, Februrary 2000.

[11] Inman, Daniel J., Vibration with Control, Measurement, and Stability Prentice Hall, New Jersey, 1989.

[12] Inman, Daniel J., Engineering Vibration Prentice Hall, New Jersey, 1996.

[13] Jordan, Peter F., Vibration Study of a Pressurized Torus Shell Part I - Experimental Study. NASA CR-884, Oct. 1967.

[14] Jordan, Peter F., Vibration and Buckling of Pressurized Torus Shells. Paper 66445, AIAA, 1966, also presented at the AIAA 4th Aerospace Sciences Meeting Los Angeles, CA June 27-29, 1966.

[15] Lassiter, John O., Engberg, Robert, Slade, Kara N., Tinker, Michael L., Comparison of Dynamic Characteristics for an Inflatable Solar Concentrator in Atmospheric and Thermal Vaccum Conditions. Paper 2000-1641, AIAA, 2000, Presented at the 41st Structures, Structural Dynamics, and Materials Conference and Exhibit, Atlanta, GA, 3-8 April 2000.

[16] Liepins, Atis A., Free Vibrations of Prestressed Toroidal Membrane. AIAA Journal, Vol3, No. 10, Oct. 1965, pp. 1924-1933.

[17] Liepins, Atis A., Flexural Vibrations of the Prestressed Toroida Shell. NASA CR-296, Sept. 1965.

[18] Liepins, Atis A., Vibration Study of a Pressurized Torus Shell Part II - Development and Applications of Analysis. NASA CR-885, Oct. 1967.

[19] Meirovitch, Leonard, Control of Structures Wiley-Interscience, New York, 1990. 
[20] Nurre, G.S., Sharkey, J.P., Nelson, J.D., and Bradley, A.J., Preservicing Mission, On-Orbit Modifications to the Hubble Space Telescope Pointing Control System. AIAA Journal of Guidance, Control, and Dynamics, Vol. 18, No. 2, Mar.-Apr. 1995, pp 222-229.

[21] Preliminary Mission Report: Spartan 207/Inflatable Antenna Experiment Flown on STS-77, Spartan Project, NASA-GSFC, Feb. 14, 1997

[22] Rao, S. and Sunar, M., Piezoelectricity and its use in disturbance sensing and control of flexible structures: A survey. Applied Mechanics Reviews, Vol. 47, No. 4, April 1994.

[23] Tinker, Michael L., Passively Adaptive Inflatable Structure for the Shooting Star Experiment. Paper 98-1986, AIAA, 1998.

[24] Williams, R.B., Experimental and Analytical Methods to Ascertain the Effects of Piezopolymer Actuators on the Dynamics of Inflatable Space-Based Structures. Virginia Tech MS Thesis, 2000. 


\section{Appendix A}

\section{Mode Shapes}

The mode shapes of toroids of two different aspect ratios are shown. The first torus has an aspect ratio of 0.04 and the second torus has an aspect ratio of 0.32 . The first fourteen modes of the small aspect ratio torus are exclusively ring modes. The first four modes of the tor us with the large aspect ratio are ring modes, but then different types of modes are manifest. Both toroids are inflated to 0.5 psi. 


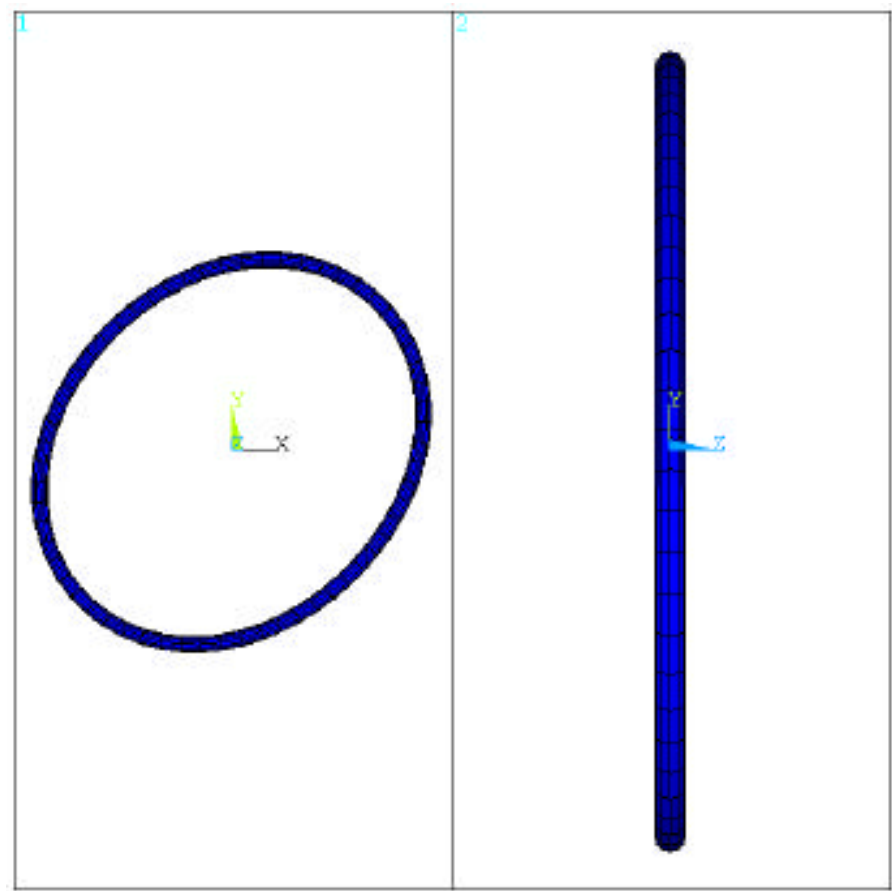

ANSSS 5.62

15.5102

TISPLACPREN

$51 \mathrm{~B}=$

PRER $=3.407$

DXCX $=5.359$

$\mathrm{DECO}=5.822$

$\mathrm{ZT}=1199,954$

$2 B=1688-04$

대IC-2 2.822

XVI $=16.16 \mathrm{~m}$

$2 \mathrm{P}=168 \mathrm{8}-04$

$\mathrm{Z}$-BSTPER

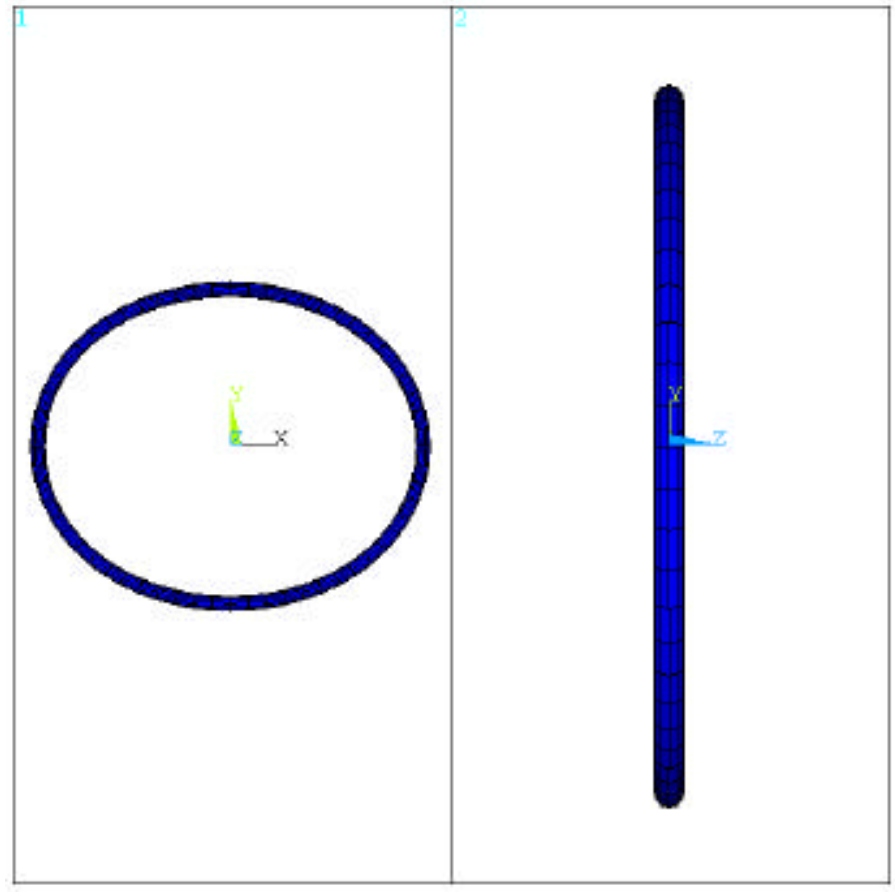

INSSYS 5.662

$15 \% 106$.

DISPLACEMEN!

$S D B=1$

PRER $=0.40$

DACX $=5.366$

$\mathrm{DECO}=5.014$

DIS1-753.611

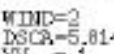

DISI $=342.28$

2-BITFER 


$$
\begin{aligned}
& \text { of } \\
& \text { of }
\end{aligned}
$$




$$
\begin{aligned}
& 0 \mid \\
& 0 \mid
\end{aligned}
$$




$$
\begin{aligned}
& \text { of } \\
& \text { of }
\end{aligned}
$$




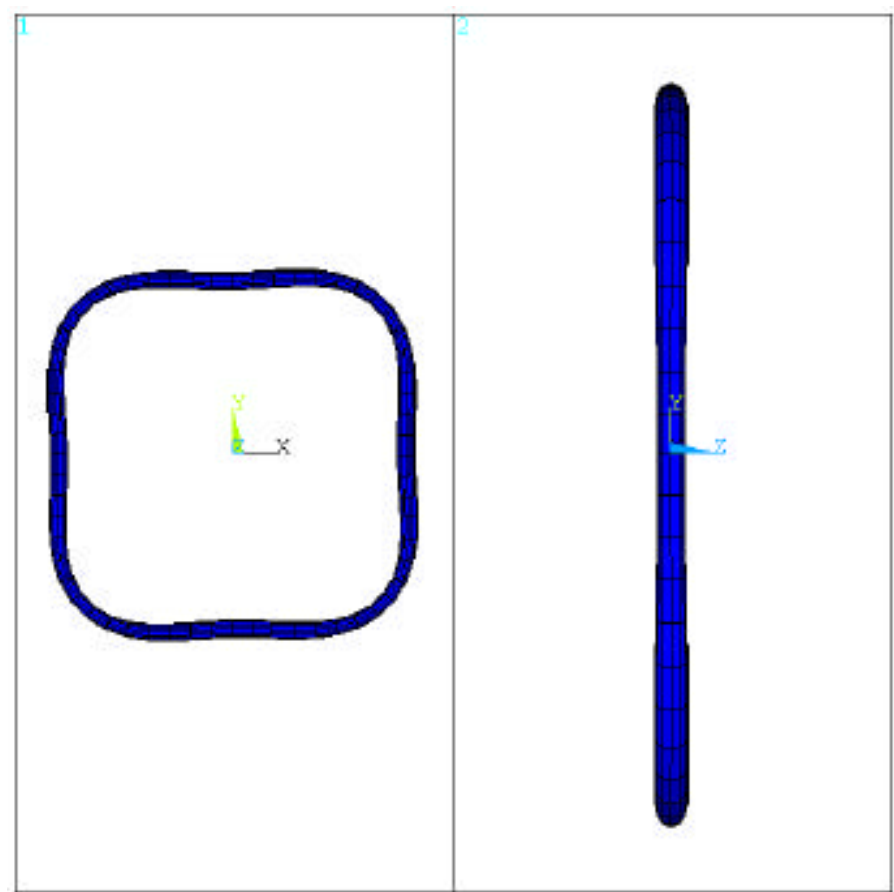

MNSSS 5.62

$15.9105,15$

MTSLACEMT

$S T B=15$

$\mathrm{FBR}=13.002$

$\mathrm{DPC}=6.039$

$\mathrm{DSCA}=5.166$

$7 y=1,1567$

$\mathrm{ZE}=2756-0.3$

패드 -2.28

XV $157-1.12281$

$=275 \mathrm{e}-0$.

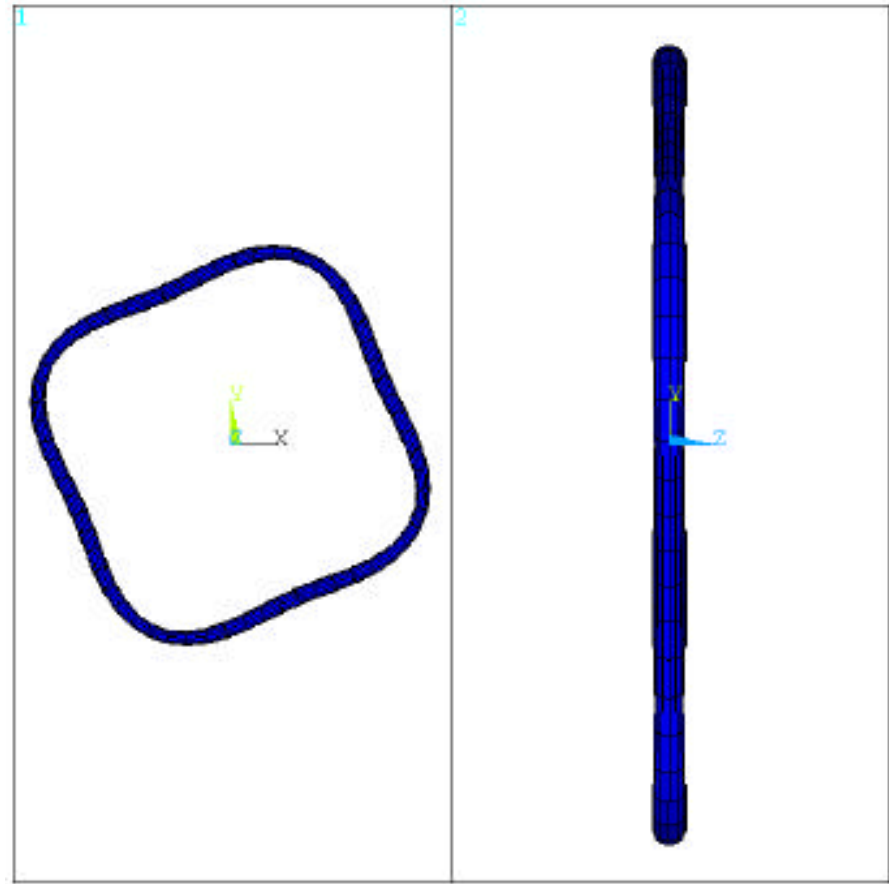

ANWSSS 5.62

15,156

TISPLACATEN

IIRe $=1$

$\mathrm{SuB}=16 \mathrm{~m}$

HIST $=0.042$

$\mathrm{DEC}=5.164$

DIFI $=113.794$

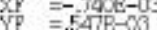

EF $=.2008-03$

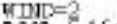

DSCA-5.164

PISI $=-159$. 394

$=54 \mathrm{E}-0 \mathrm{~s}$

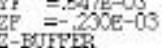




$$
\begin{aligned}
& 0\} \\
& 0 \& 5
\end{aligned}
$$




$$
\begin{aligned}
& 01 \\
& 01
\end{aligned}
$$



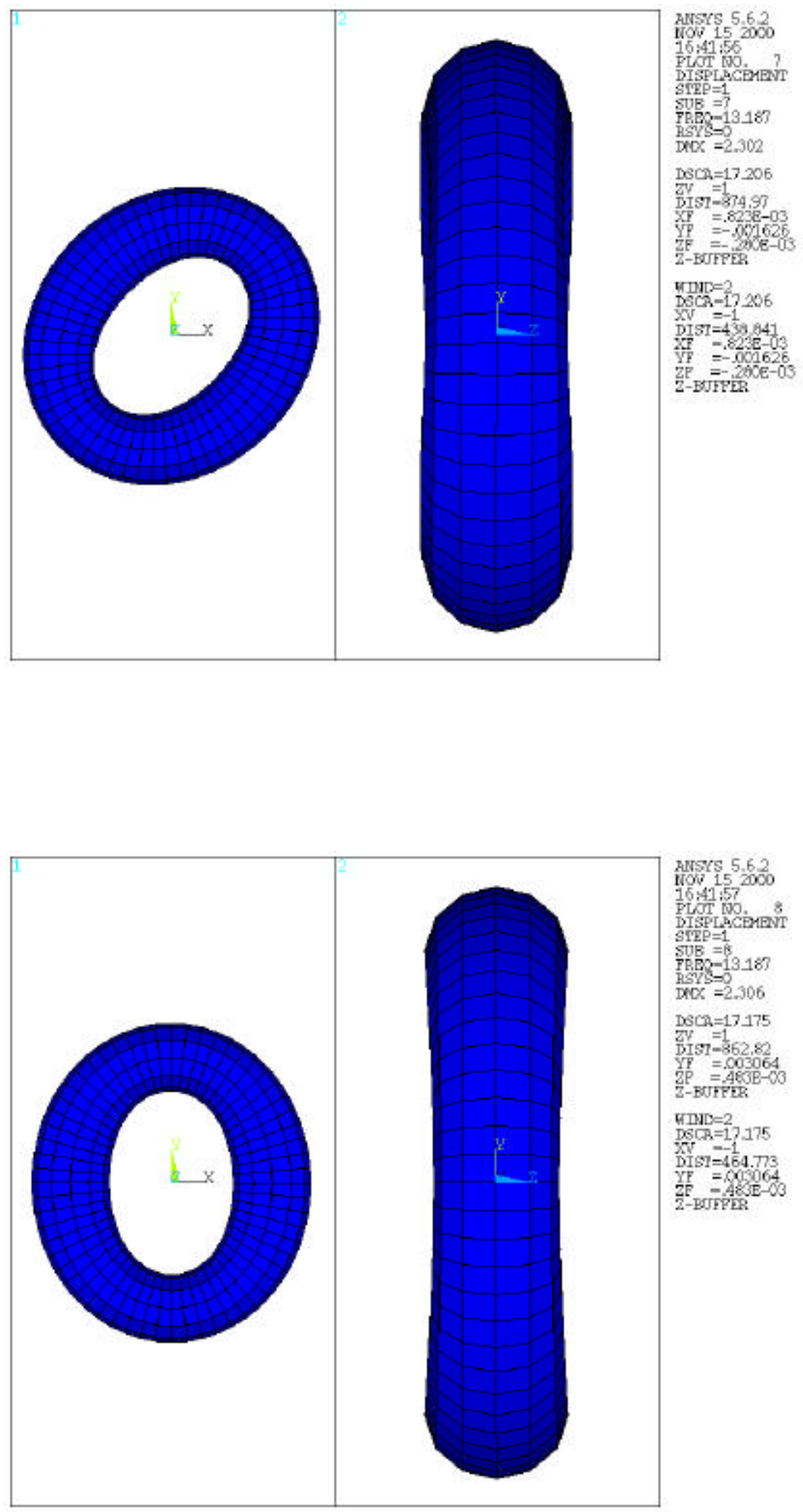

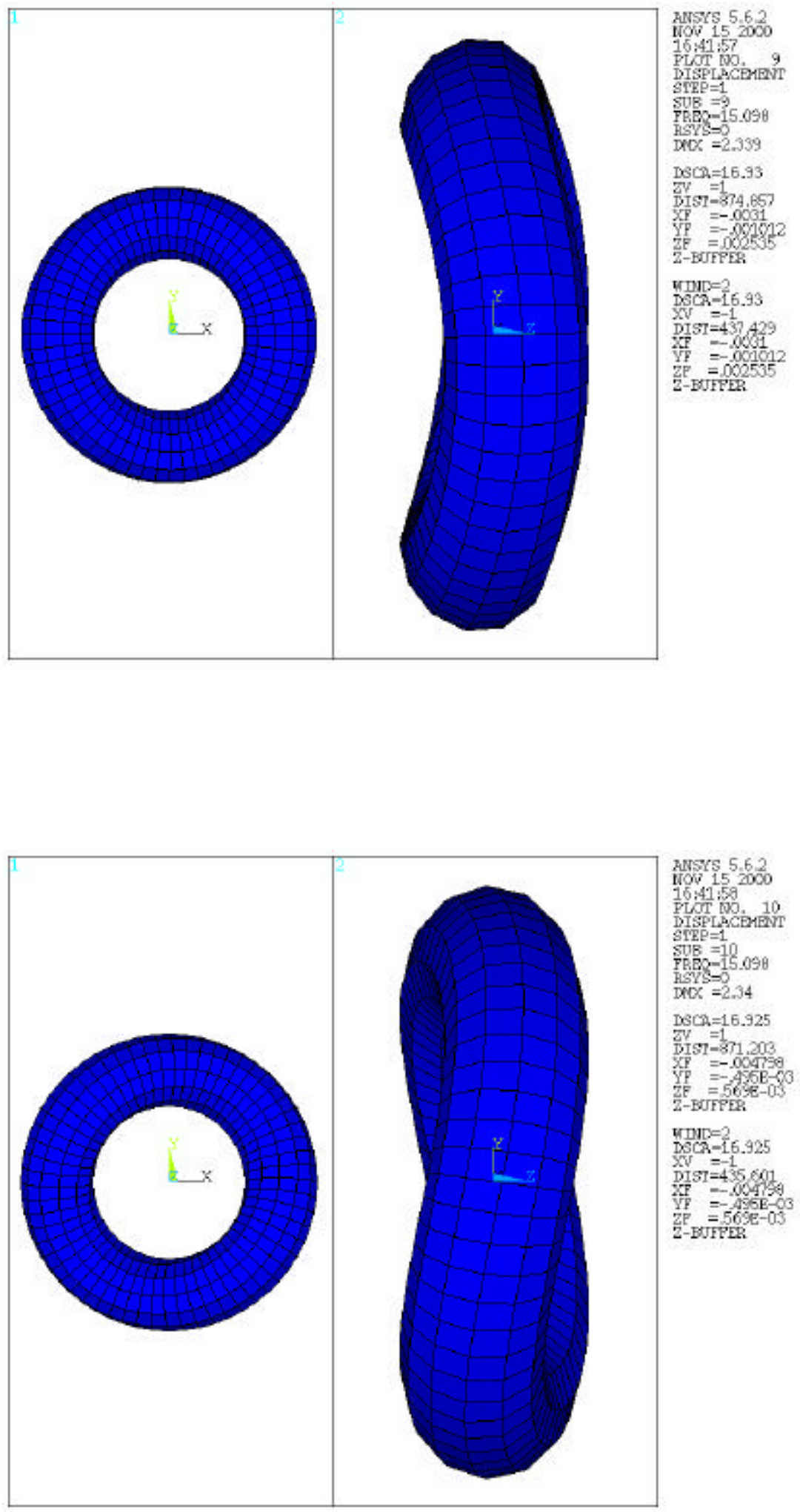

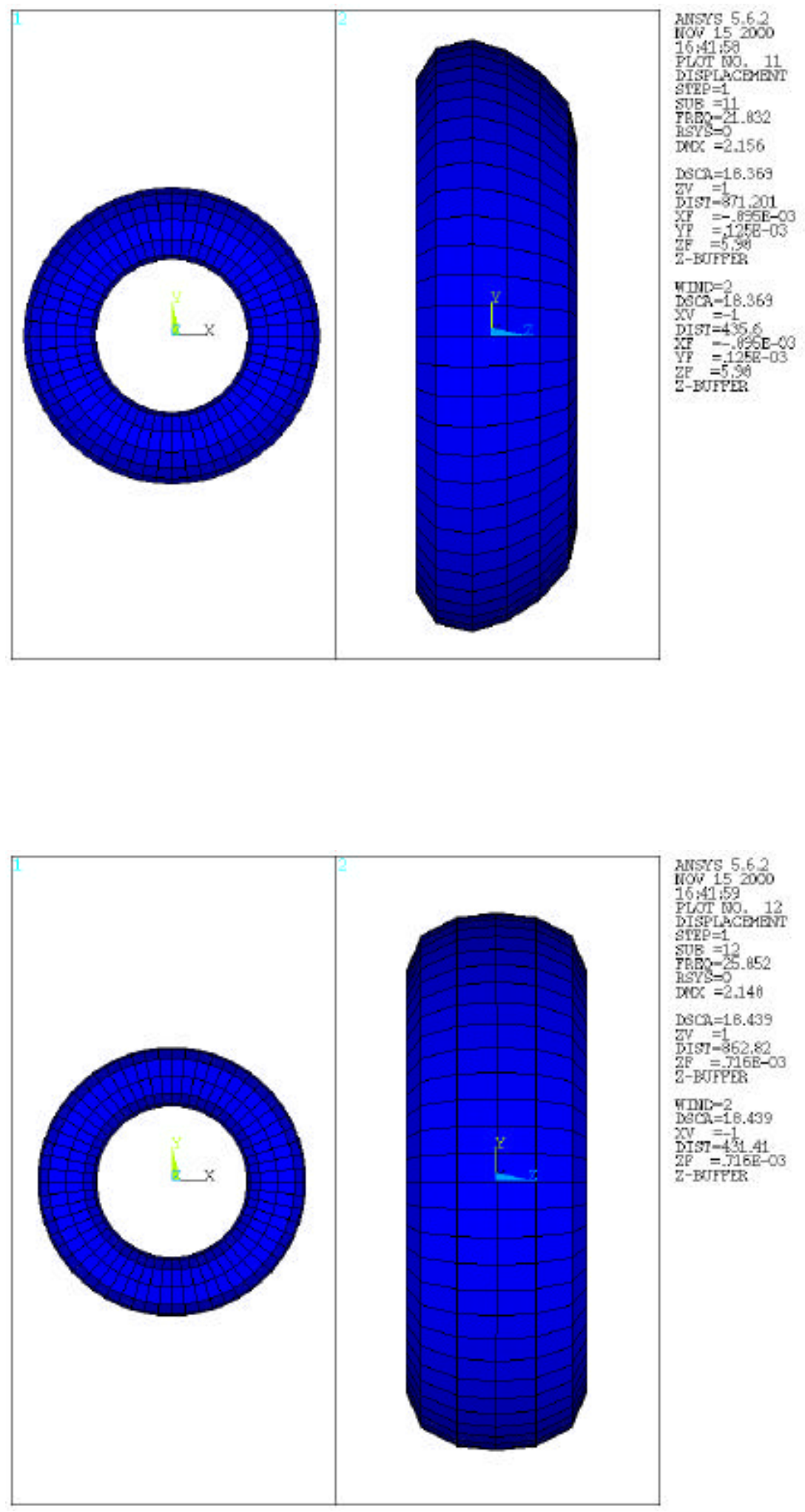

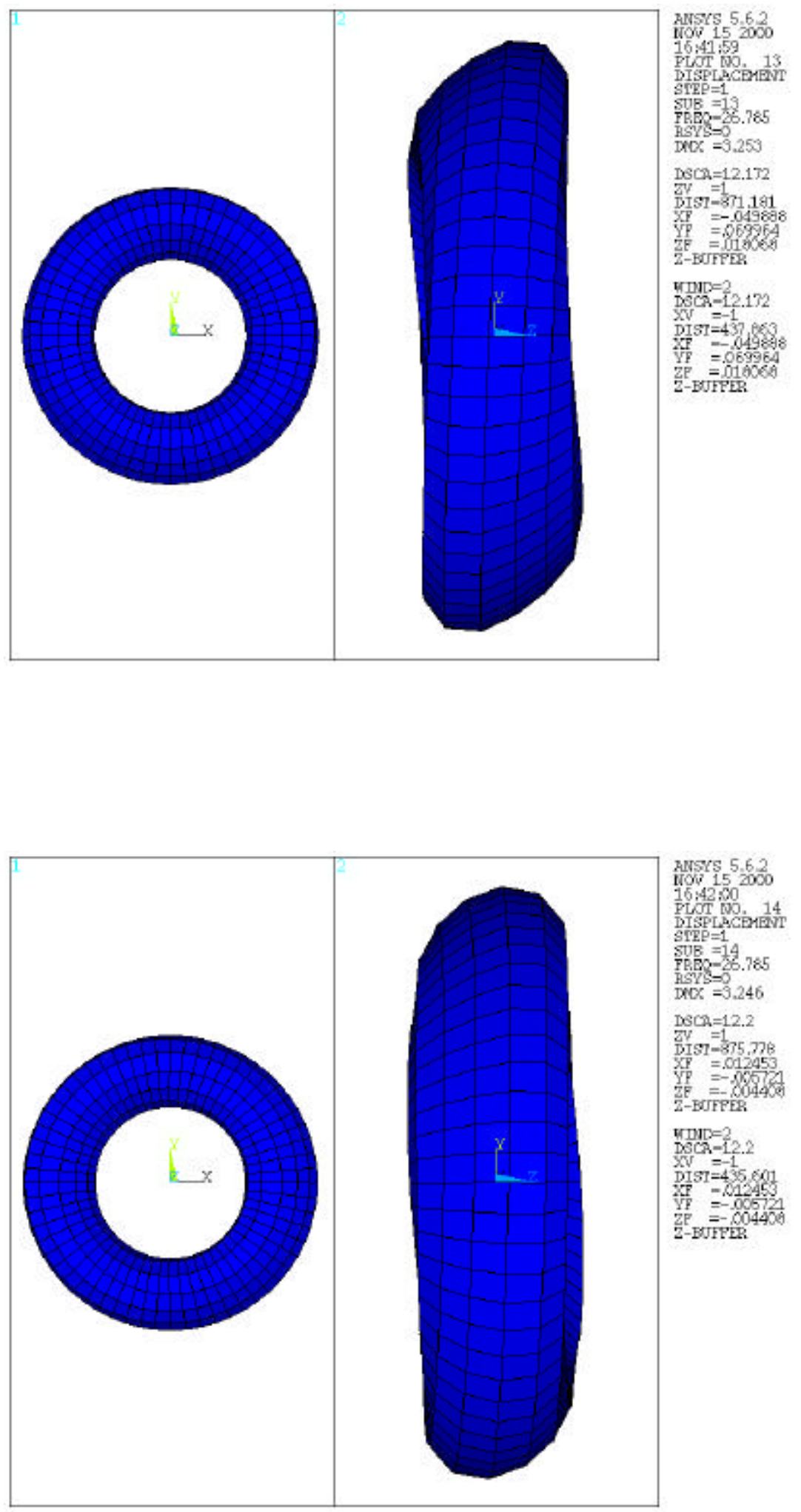

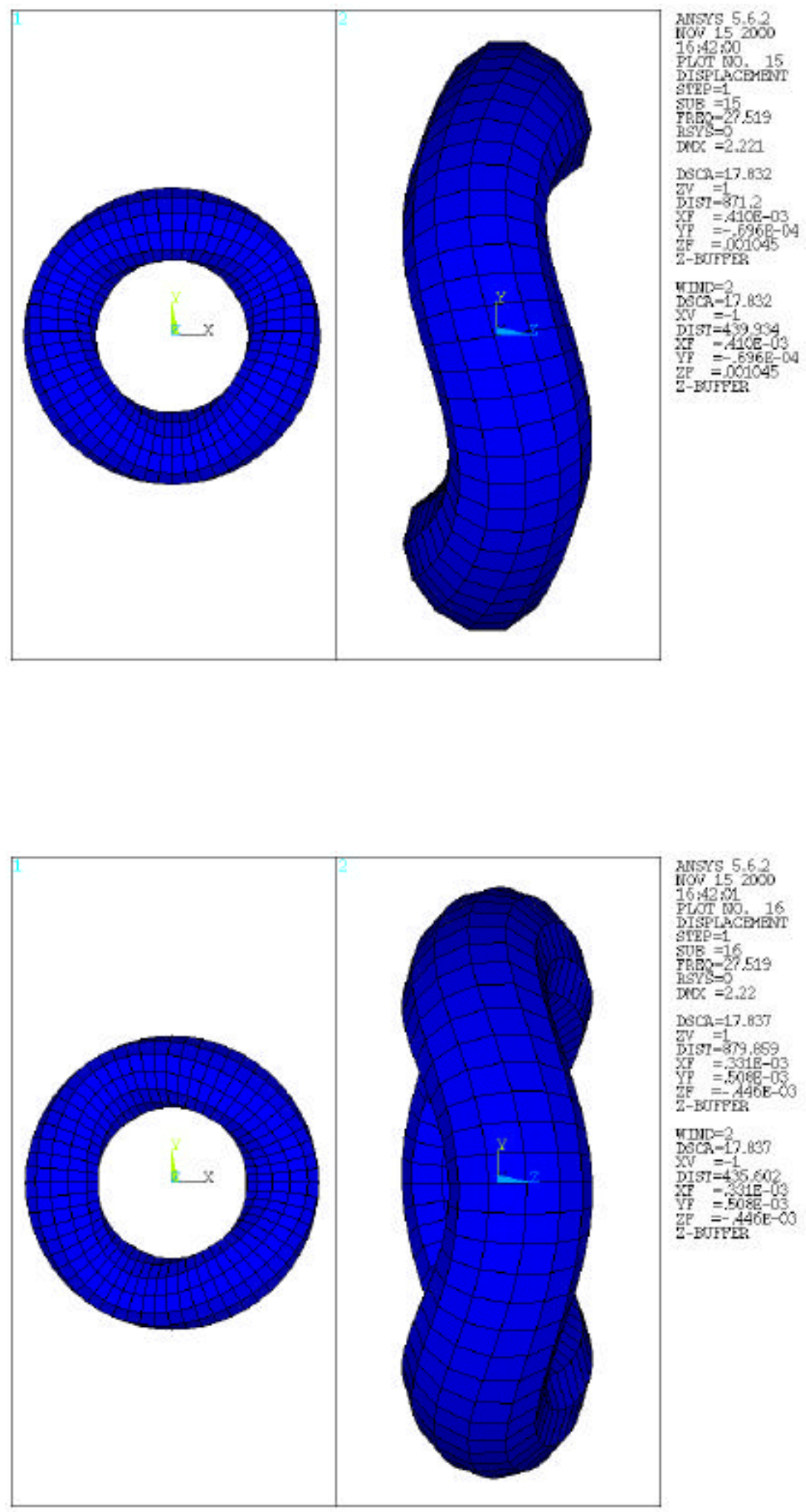

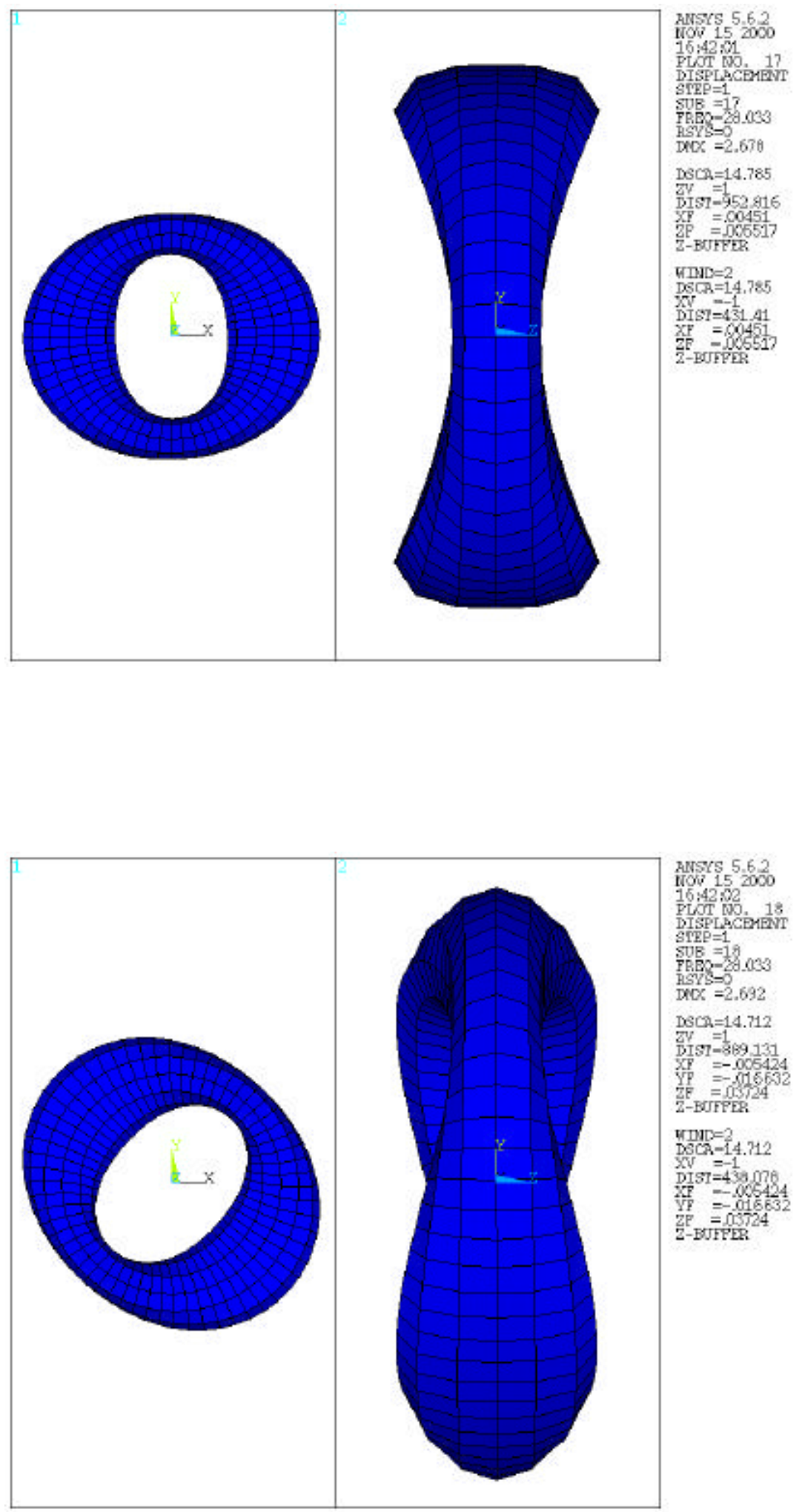

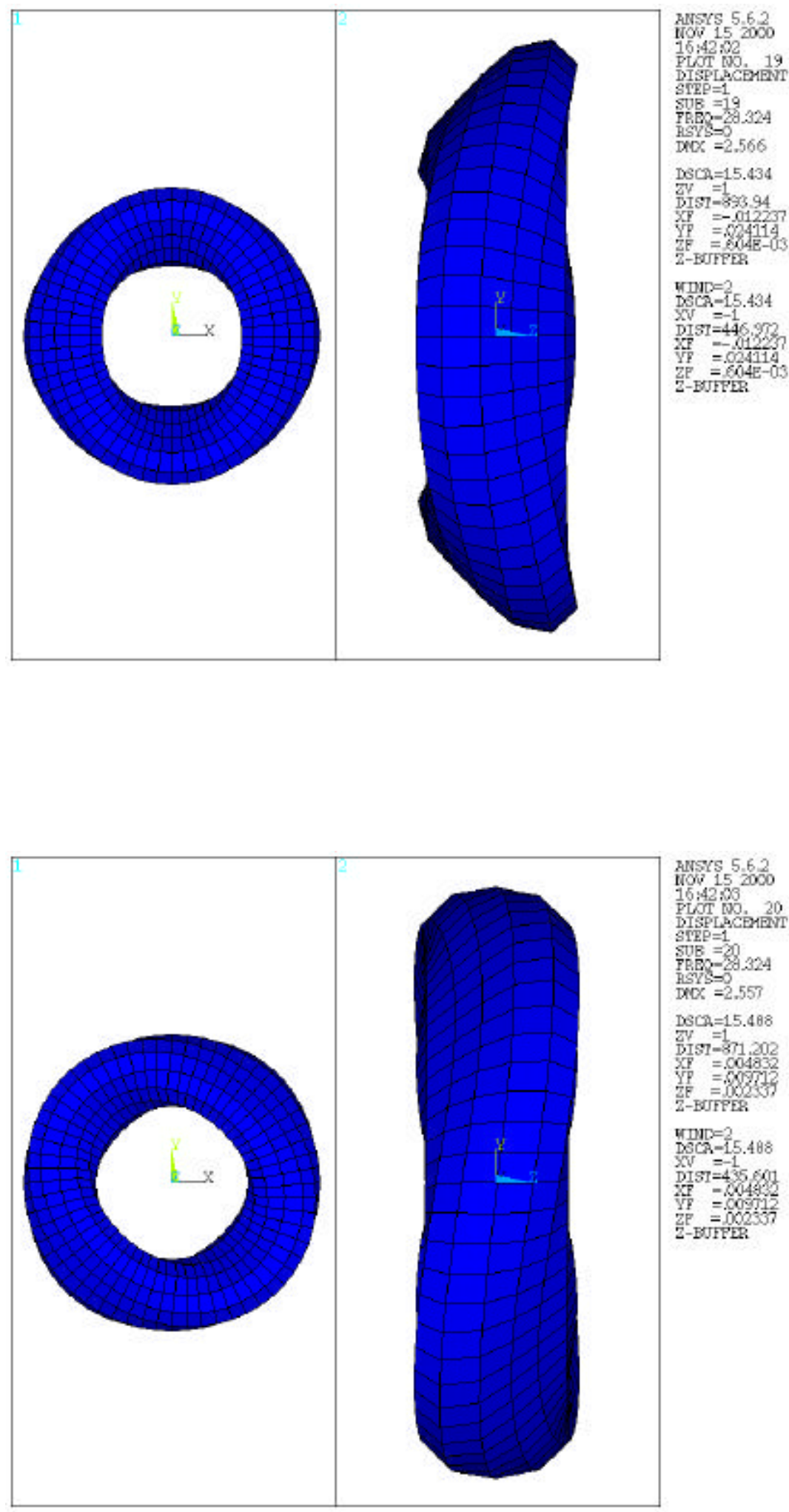


\section{Appendix B}

\section{MATLAB Mesh Generation}

$\%$ Generate node locations $(x, y, z)$ and connectivity for a torus

$\%$ with major radius $\mathrm{R} O$ and minor radius $\mathrm{r} O$.

$\%$ The outputs of this file elem8.csv and node.csv can be manipulated

$\%$ in MS Excel to form appropraite input files to ANSYS.

$\%$ The output files are already in comma delimited format which is

$\%$ what ANSYS needs. Therefore just open them in Excel, edit the files

$\%$ and then save them again as comma delimited format.

$\%$ The node.csv file needs to be in the form:

$\% \mathrm{n}$, ,\#, \#,\#

$\%$ This is accomplished by adding two columns to the worksheet in Excel

$\%$ and then filling the first with "n"s. Also, zero values are output

$\%$ as blanks so the three columns with numbers (only including the rows

$\%$ of interest) need to be highlighted and the replace all command needs

$\%$ to be used, replacing all blank values with "0"s.

$\%$ The elem8.csv file needs to be in the form:

$\%$ e,\#,\#,\#,\#,\#,\#,\#,\#

$\%$ This is accomplished by adding one column to the worksheet in Excel

$\%$ and then filling it with "e"s.

$\%$ Jackson A. Lewis 12/00

$\%$ Originally Written by Eric M. Austin. 9/22/99

clear all

close all

$\%$ Number of elements in the circumferencial direction (angle eta), i.e., $\%$ around the large, outer circle of the torus 


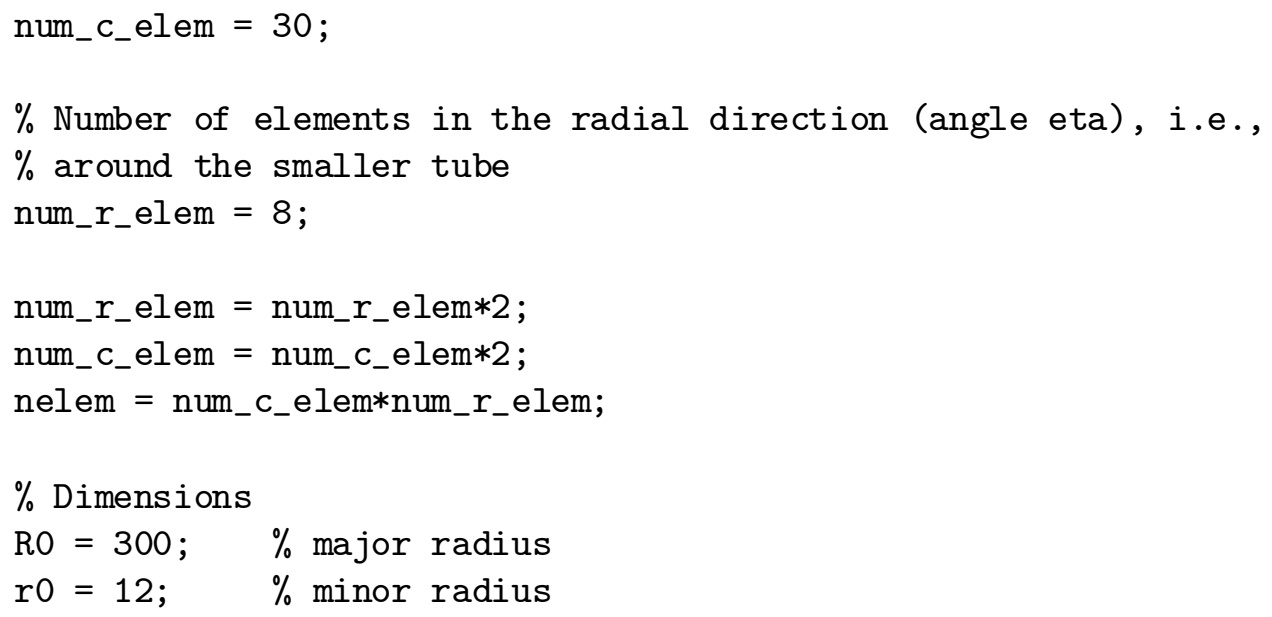

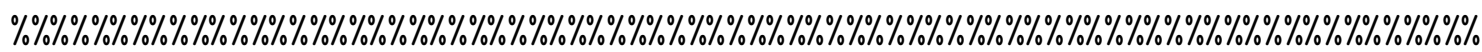

$\%$ Build the mesh as a flat domain and match up node numbers at the end.

$\%$ This is a single strip $(0 \rightarrow 2 \mathrm{pi})$ around the outer diameter of the torus.

$\%$ The ends are matched up, so this is a complete hoop.

basic_strip $=\left[\left(1:\right.\right.$ num_c_elem $^{\prime},, \ldots$

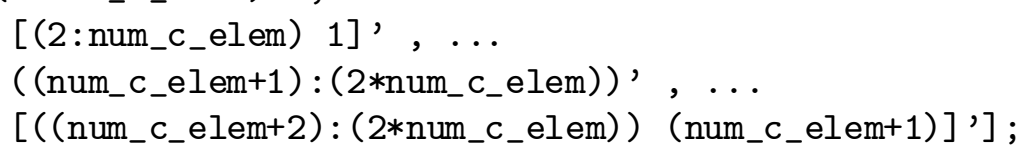

$\%$ dimension the connectivity matrix

con $=$ zeros (num_r_elem*num_c_elem,4);

$\%$ Duplicate the "basic_strip" above for each strip around the smaller tube.

for ir $=1$ :num_r_elem $\operatorname{con}\left(\left((i r-1) * n u m_{-} c_{-} e l e m+1\right):\right.$ ir $*$ num_c_elem, $\left.:\right)=($ ir -1$) * n u m_{-} c_{-} e l e m+$ basic_strip; end

$\%$ Connect the last row to the first

con $((($ num_r_elem-1) $*$ num_c_elem+1) :num_r_elem*num_c_elem, 3:4) = basic_strip $(:, 1: 2)$;

con2=zeros (num_r_elem*num_c_elem/4,8);

for $i=0:$ num_c_elem/2-1

for $j=0:$ num_r_elem $/ 2-1$ $\operatorname{con} 2\left((i+1)+\left(j * n u m_{-} c_{-} e l e m / 2\right),:\right)=\left[\operatorname{con}\left(2 * j * n u m_{-} c_{-} e l e m+2 * i+1,1\right), \ldots\right.$ con $(2 * j *$ num_c_elem $+2 * i+2,2), \ldots$ $\operatorname{con}\left((2 * j+1) * n u m_{-} c_{-} e l e m+2 * i+2,4\right), \operatorname{con}\left((2 * j+1) * n u m_{-} c_{-} e l e m+2 * i+1,3\right), \ldots$ con $(2 * j *$ num_c_elem $+2 * i+2,1), \operatorname{con}(2 * j *$ num_c_elem $+2 * i+2,4), \ldots$ $\left.\operatorname{con}\left((2 * j+1) * n u m_{-} c_{-} e l e m+2 * i+1,4\right), \operatorname{con}\left((2 * j+1) * n u m_{-} c_{-} e l e m+2 * i+1,1\right)\right]$;

end

end 


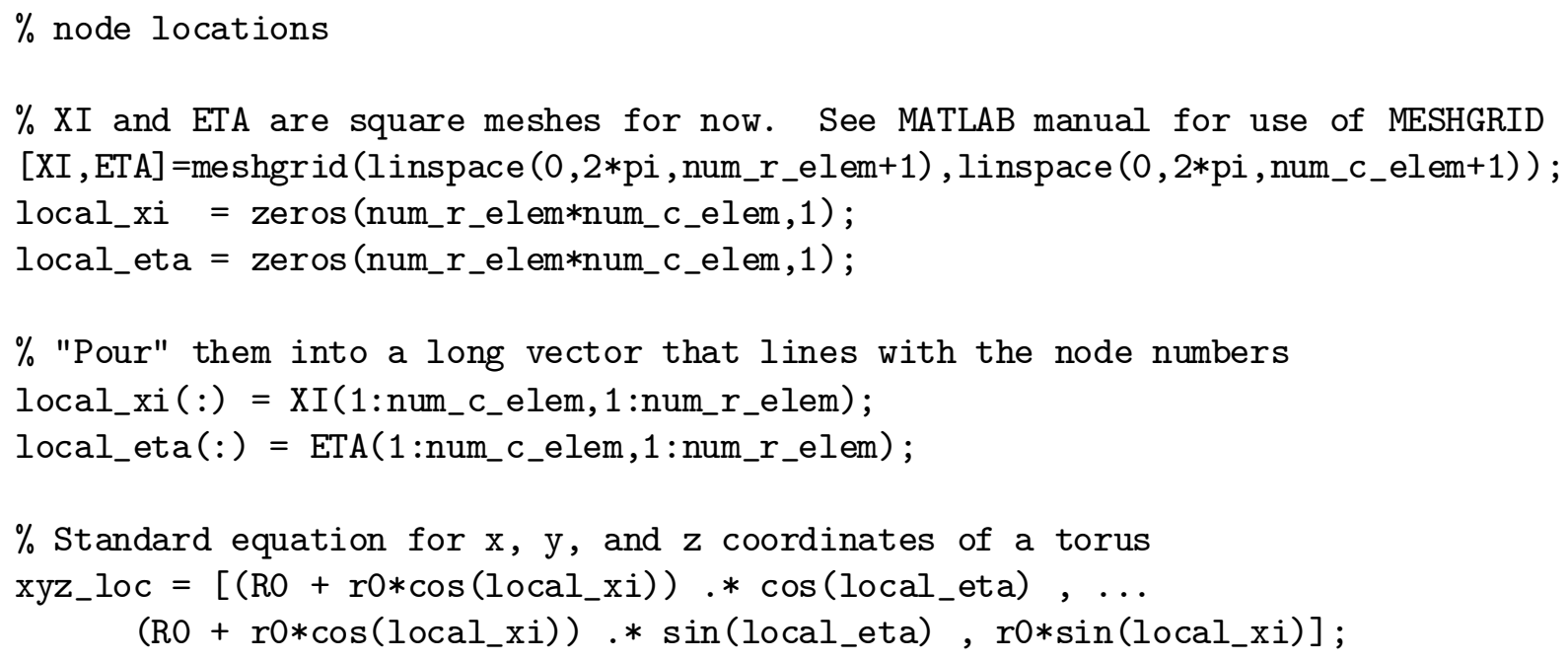


axis off

$\%$ Format for Ansys

cona $=[\operatorname{con}(:, 1), \operatorname{con}(:, 2), \operatorname{con}(:, 4), \operatorname{con}(:, 3)]$;

dlmwrite ('elem4.csv', cona)

dlmwrite ('elem8.csv', con2)

dlmwrite ('node.csv', xyz_loc) 


\title{
Appendix $\mathrm{C}$
}

\section{MATLAB Eigenvalue Solution}

\author{
\% Solve Eigenvalue Problem \\ $\%$ Written By Jackson A. Lewis 12/00 \\ clear all \\ close all \\ $\%$ Set Variables \\ $f=1 e-6$; \\ $\operatorname{dof} \mathrm{s}=6$; \\ \%Magnitude of Forcing Function \\ $\%$ ndof $=6000 * 6$; \%Ttal Degrees of Freedom in Model \\ criteria=0; \%Criteria for guyan reduced DOF \\ $\% .001$ means remove DOFS smaller than .001*largest \\ $\% 0$ means remove only massless dofs \\ nmcalc $=30 ; \quad \%$ Number of Eigenvalues to Calculate \\ nmodes $=10 ; \quad \%$ Number of Modes to Retain in modal truncation \\ $\%$ Must be $<=$ nmcalc \\ $\% \% \% \% \% \% \% \% \% \% \% \% \% \% \% \% \% \% \% \% \% \% \% \% \% \% \% \% \% \% \% \% \% \% \% \% \% \% \% \% \% \% \% \% \% \% \% \% \% \% \% \% \% \% \% \% \% \% \% \% \% \% \% \% \%$ \\ \%\%\%\%\%\%\%\%\%\%\%\%\%\%\%\%\%\%\%\%\%\%\%\%\%\%\%\%\%\%\%\%\%\%\%\%\%\%\%\%\%\%\%\%\%\%\%\%\%\%\%\%\%\%\%\%\%\%\%\%\%\%\%\%\%\%\%\%\% \\ $\%$ Read in unformatted mass and stiffness matrices \\ [m1 , m2 , m3] =textread ('mass.txt' , '\%u \%u \%f') ; \\ $\mathrm{m}=[\mathrm{m} 1, \mathrm{~m} 2, \mathrm{~m} 3]$; \\ $[\mathrm{k} 1, \mathrm{k} 2, \mathrm{k} 3]=$ textread ('stiff.txt' , ' $\% \mathrm{u} \% \mathrm{u} \% \mathrm{f}$ ') ; \\ $\mathrm{k}=[\mathrm{k} 1, \mathrm{k} 2, \mathrm{k} 3]$; \\ $\%$ Number of DOF in Model \\ ndofm $=\max ([\max (m(:, 1)), \max (m(:, 2))])$; \\ $n$ dof $k=\max ([\max (k(:, 1)), \max (m(:, 2))])$; \\ ndof $c=\max$ (ndofm,ndof $k$ ); \\ ndof $c=$ dof $s * \operatorname{ceil}$ (ndofc/dofs); \\ $\%$ check $=$ ndof - ndofc \\ ndof $=$ ndo $f c$
}




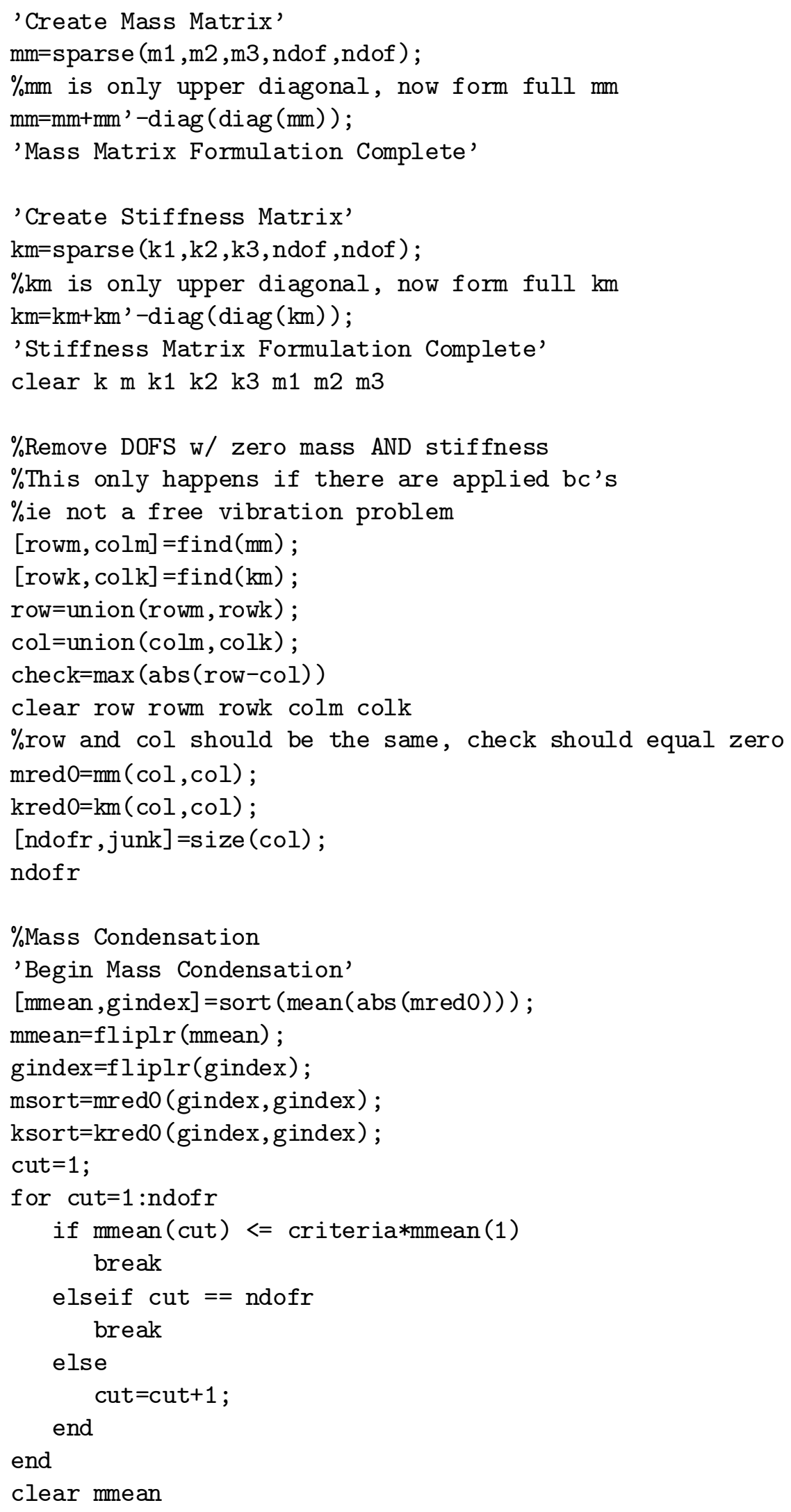




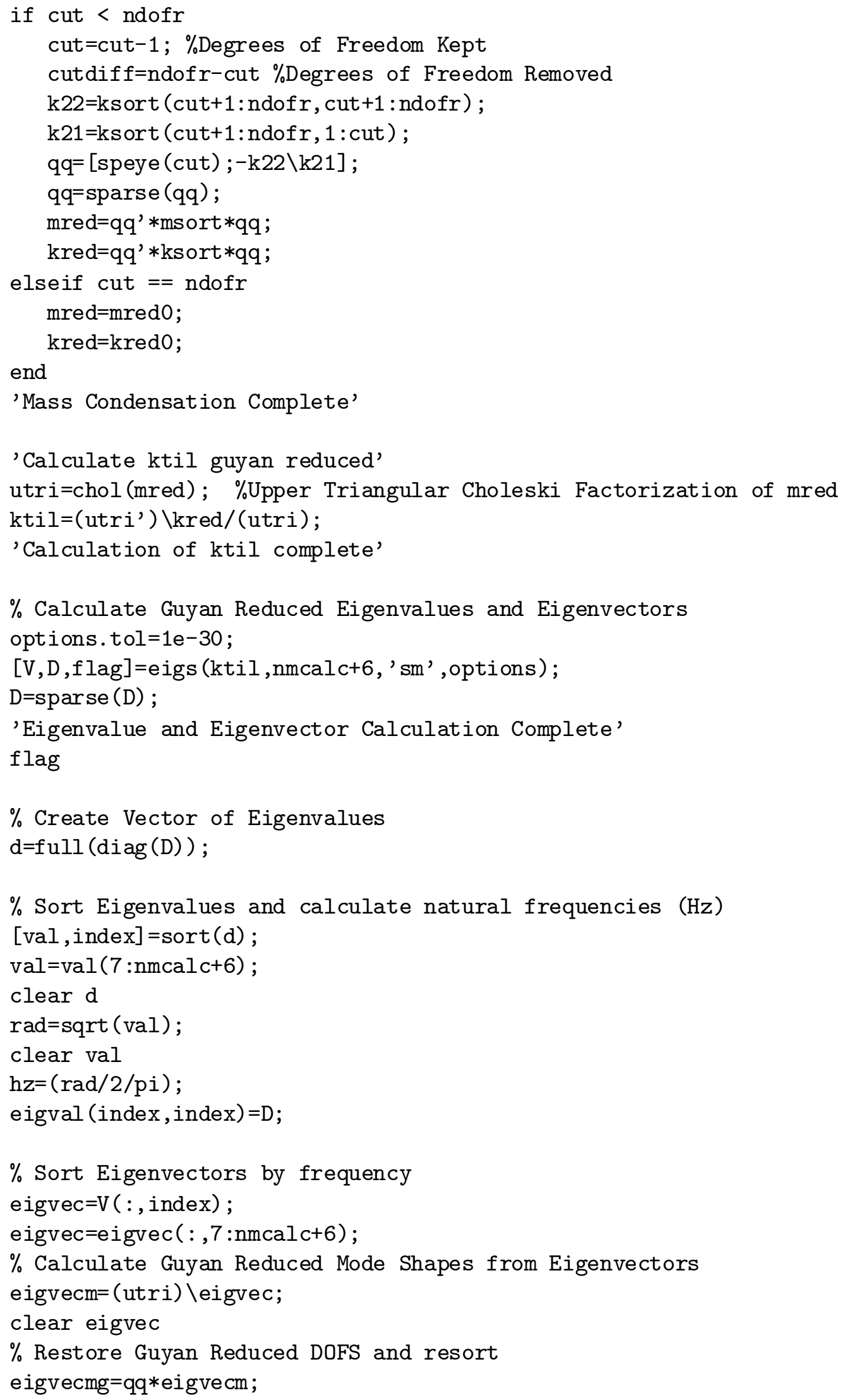




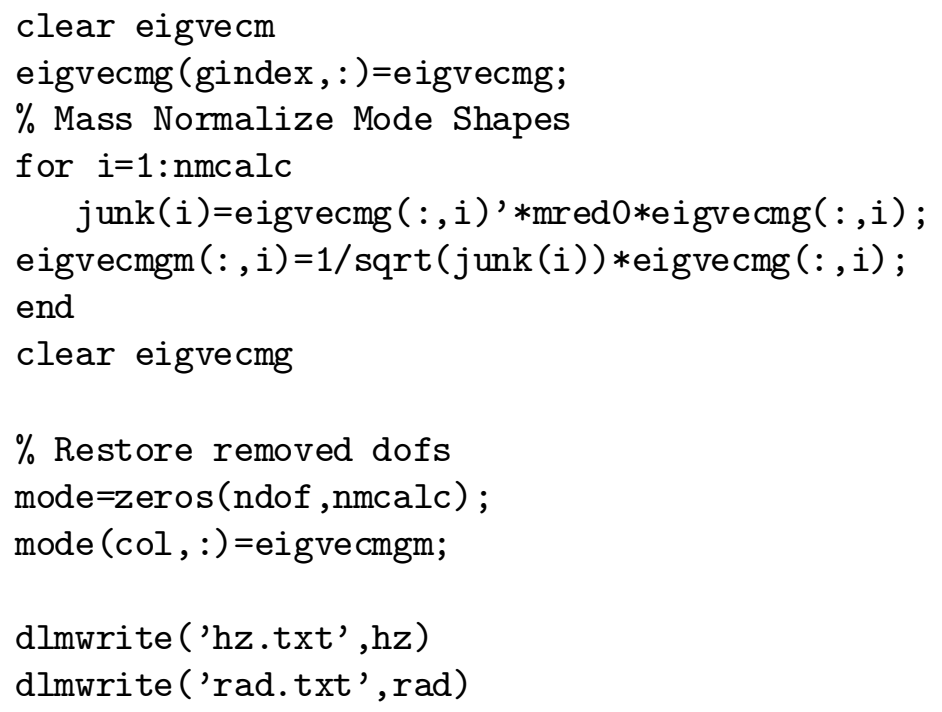




\title{
Appendix D
}

\section{MATLAB Mode Shape Post Processor}

\author{
$\%$ Post Process Mode Shapes \\ $\%$ Written By Jackson A. Lewis 12/00 \\ close all \\ mplot=1 \%ode number to plot \\ dscale $=10 \quad \%$ Scale Factor for Mode Shape Plot

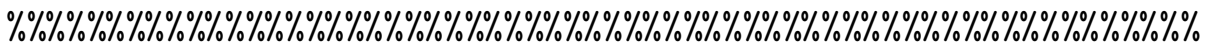

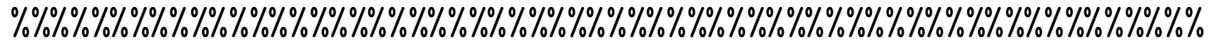 \\ $\%$ 3D matrix that is [Nodes X Degrees of Freedom Each Node X Mode Shapes] \\ 'Calculate Mode Shape Matrix' \\ disp=zeros (ceil (ndof/dofs), dof s,nmcalc); \\ for $i=1$ : ceil (ndof/dofs) \\ for $j=1$ :dofs \\ $\operatorname{displ}(i, j,:)=\operatorname{mode}(\operatorname{dof} s * i-(\operatorname{dofs}-j),:)$; \\ end \\ end

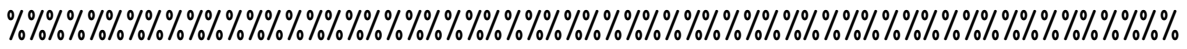 \\ $\% \% \% \% \% \% \% \% \% \% \% \% \% \% \% \% \% \% \% \% \% \% \% \% \% \% \% \% \% \% \% \% \% \% \% \% \% \% \% \% \% \% \% \% \% \% \% \% \% \% \% \% \% \% \% \% \%$ \\ $\% \%$ Created in Part by Eric Austin \\ $\%$ Post Processing of Mode Shapes \\ $\%$ Plot Torus undeformed and modeshape specified by mplot above \\ $\%$ Number of elements in the circumferencial direction (angle eta), i.e., \\ $\%$ around the large, outer circle of the torus \\ num_c_elem $=30$; \\ $\%$ Number of elements in the radial direction (angle eta), i.e.,
}


$\%$ around the smaller tube

num_r_elem = 8;

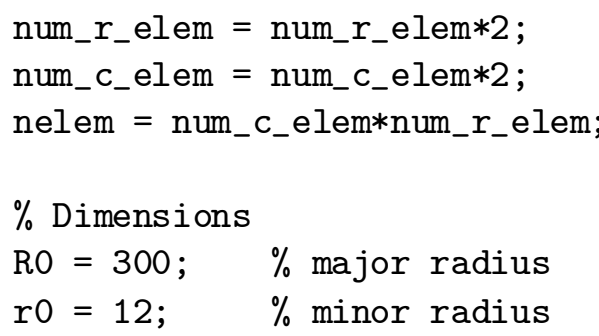

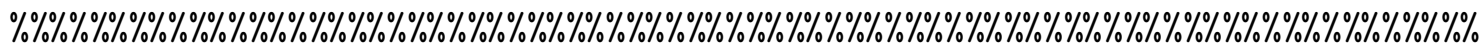

$\%$ Build the mesh as a flat domain and match up node numbers at the end.

$\%$ This is a single strip ( $0 \rightarrow 2 \mathrm{pi}$ ) around the outer diameter of the torus.

$\%$ The ends are matched up, so this is a complete hoop.

basic_strip $=[(1:$ num_c_elem $),, \ldots$

$[(2:$ num_c_elem $) 1],, \ldots$

$\left(\left(\right.\right.$ num_c_elem+1) $\left.:\left(2 * n u m_{-} c_{-} e l e m\right)\right),, \ldots$

$\left.\left[\left((\text { num_c_elem }+2):\left(2 * n u m \_c_{-} e l e m\right)\right) \text { (num_c_elem+1) }\right]^{\prime}\right]$;

$\%$ dimension the connectivity matrix

con $=$ zeros (num_r_elem $*$ num_c_elem, 4$)$;

$\%$ Duplicate the "basic_strip" above for each strip around the smaller tube.

for ir $=1$ :num_r_elem

con $\left(\left((i r-1) * n u m_{-} c_{-} e l e m+1\right):\right.$ ir $*$ num_c_elem,$\left.:\right)=\ldots$

(ir-1)*num_c_elem + basic_strip;

end

$\%$ Connect the last row to the first

con ( ( $($ num_r_elem-1)*num_c_elem+1) : num_r_elem*num_c_elem, $3: 4)=\ldots$

basic_strip $(:, 1: 2)$;

con2=zeros (num_r_elem*num_c_elem/4,8);

clear $j$;

clear $i$;

for $i=0:$ num_c_elem/2-1

for $j=0:$ num_r_elem $/ 2-1$

$\operatorname{con} 2((i+1)+(j *$ num_c_elem/2),$:)=\ldots$

$[\mathrm{con}(2 * j *$ num_c_elem $+2 * i+1,1), \ldots$

$\operatorname{con}\left(2 * j *\right.$ num $c_{-}$elem $\left.+2 * i+2,2\right), \ldots$

$\operatorname{con}\left((2 * j+1) * n u m_{-} c_{-} e l e m+2 * i+2,4\right), \ldots$

$\operatorname{con}((2 * j+1) *$ num_c_elem $+2 * i+1,3), \ldots$

con $(2 * j *$ num_c_elem $+2 * i+2,1), \ldots$ 


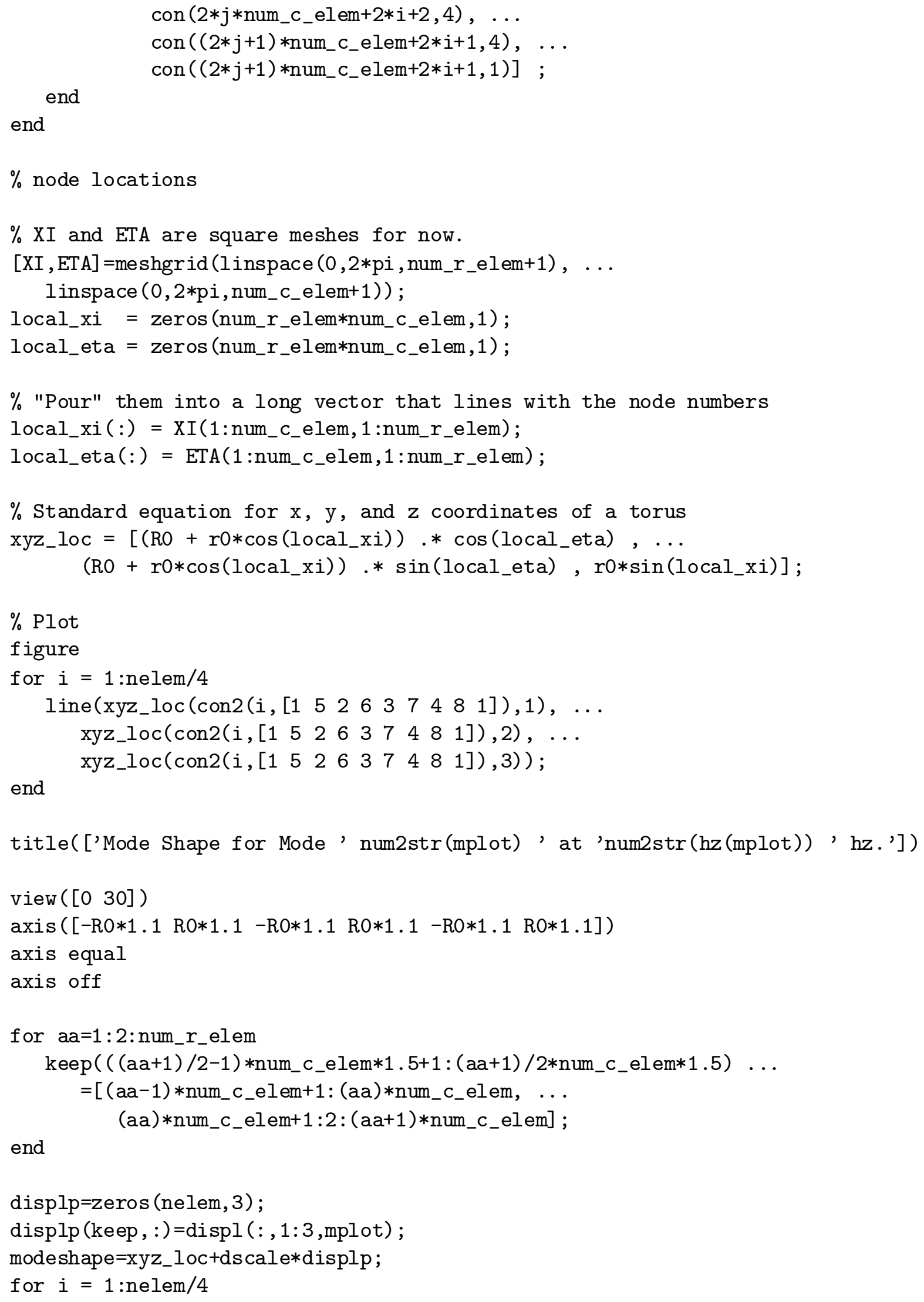

end

displp=zeros (nelem, 3);

displp (keep, : )=displ (: , 1:3,mplot);

modeshape $=x y z \_l o c+d s c a l e * d i s p l p$;

for $i=1: \mathrm{nelem} / 4$ 


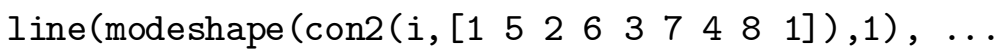

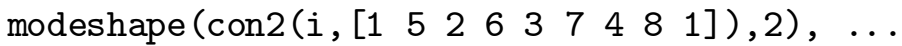
modeshape (con2(i, [1 $\left.\left.\begin{array}{lllllllll}1 & 5 & 2 & 6 & 3 & 7 & 4 & 8 & 1\end{array}\right]\right)$, 3), 'color', 'r') ;

end 


\section{Appendix E}

\section{MATLAB Settle Time Calculation}

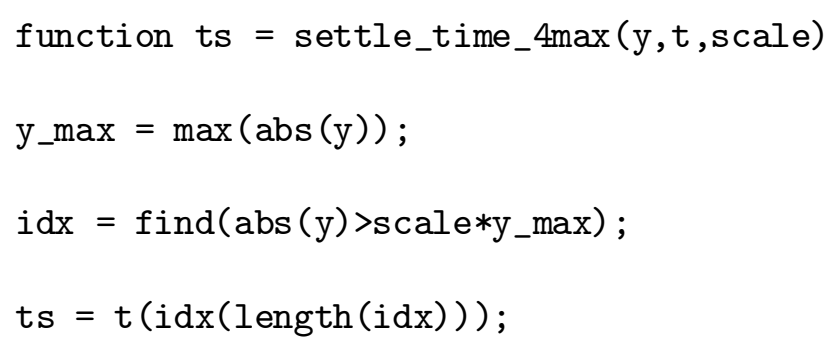




\section{Appendix F}

\section{MATLAB Observer Feedback Simulation}

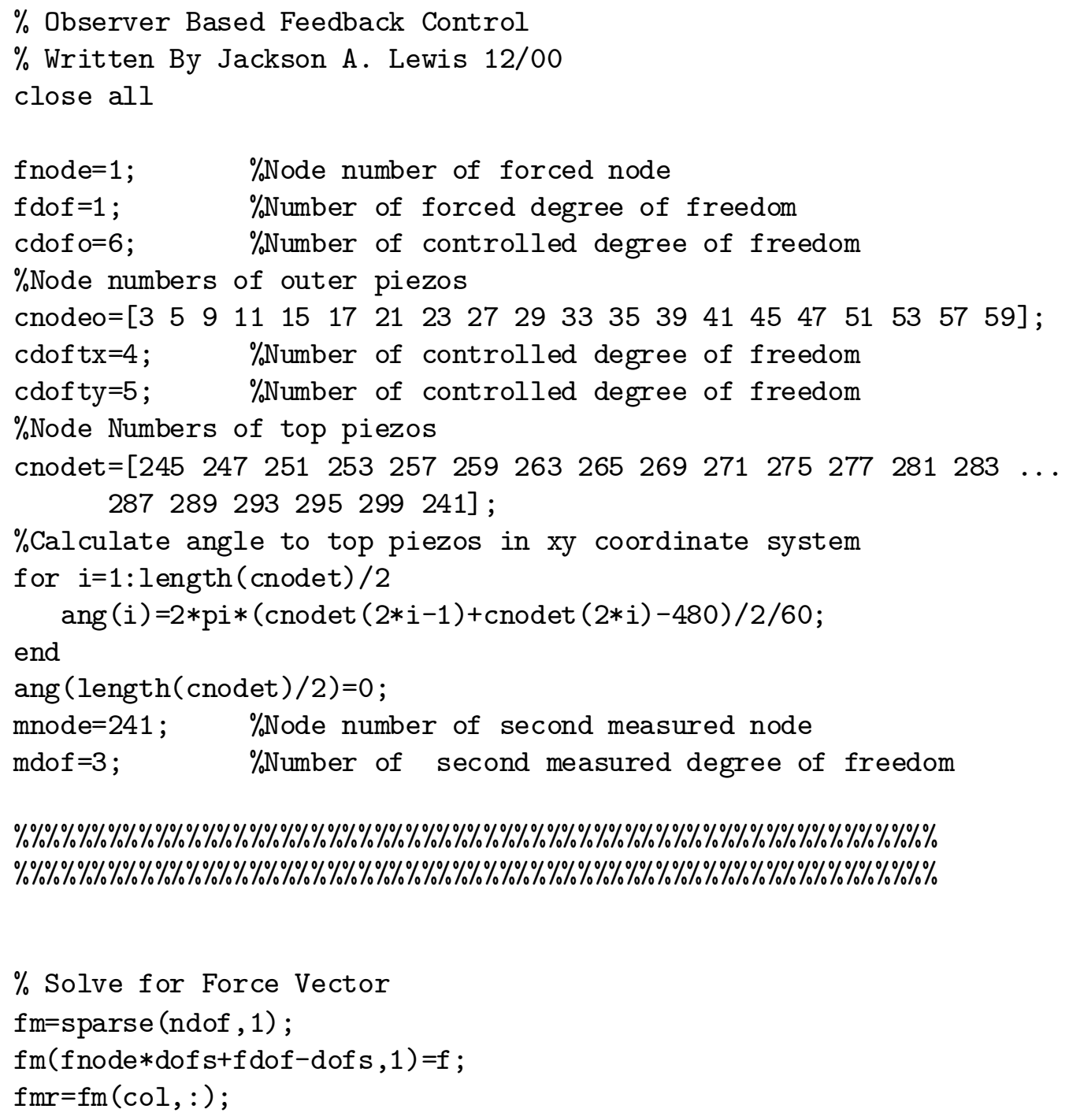




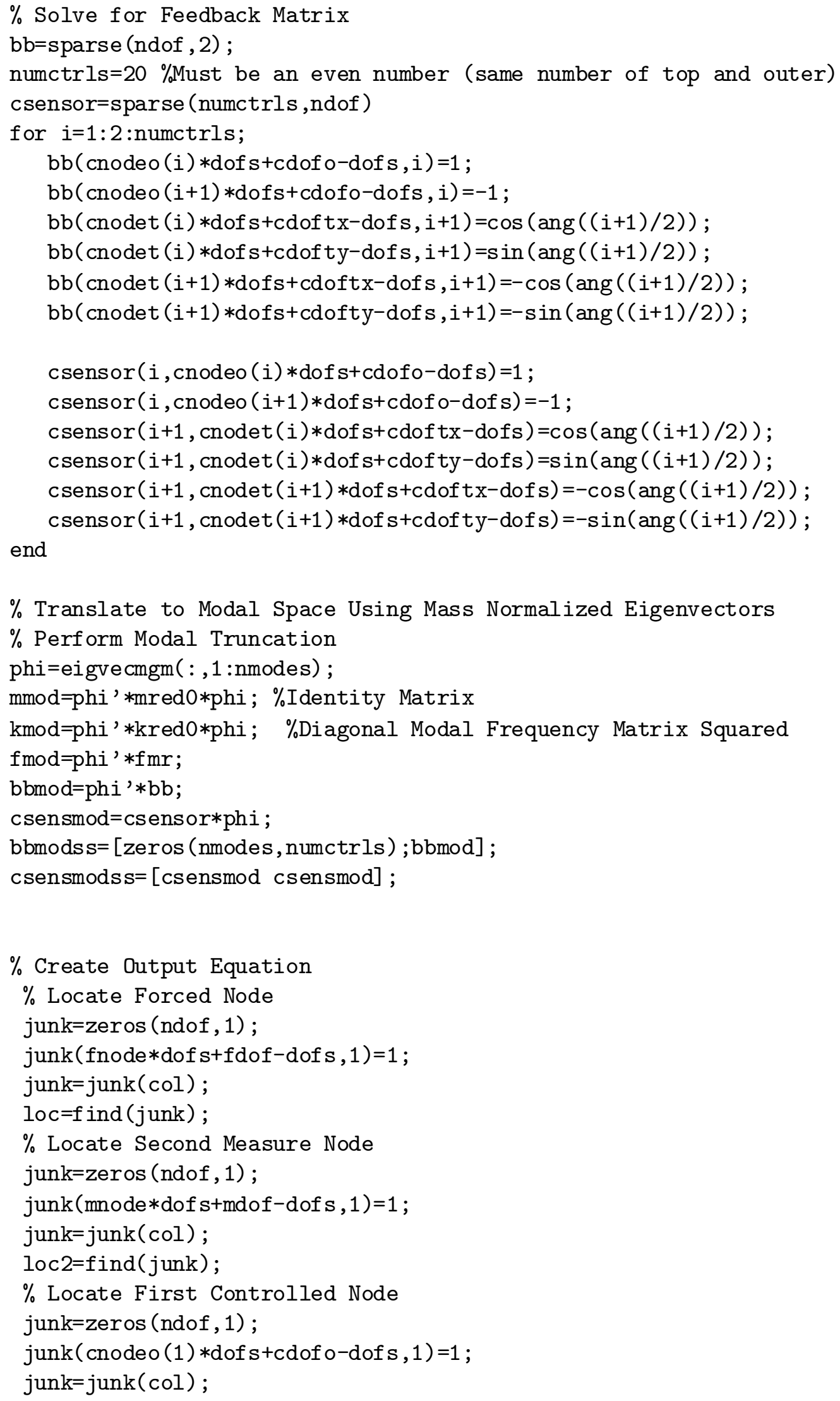









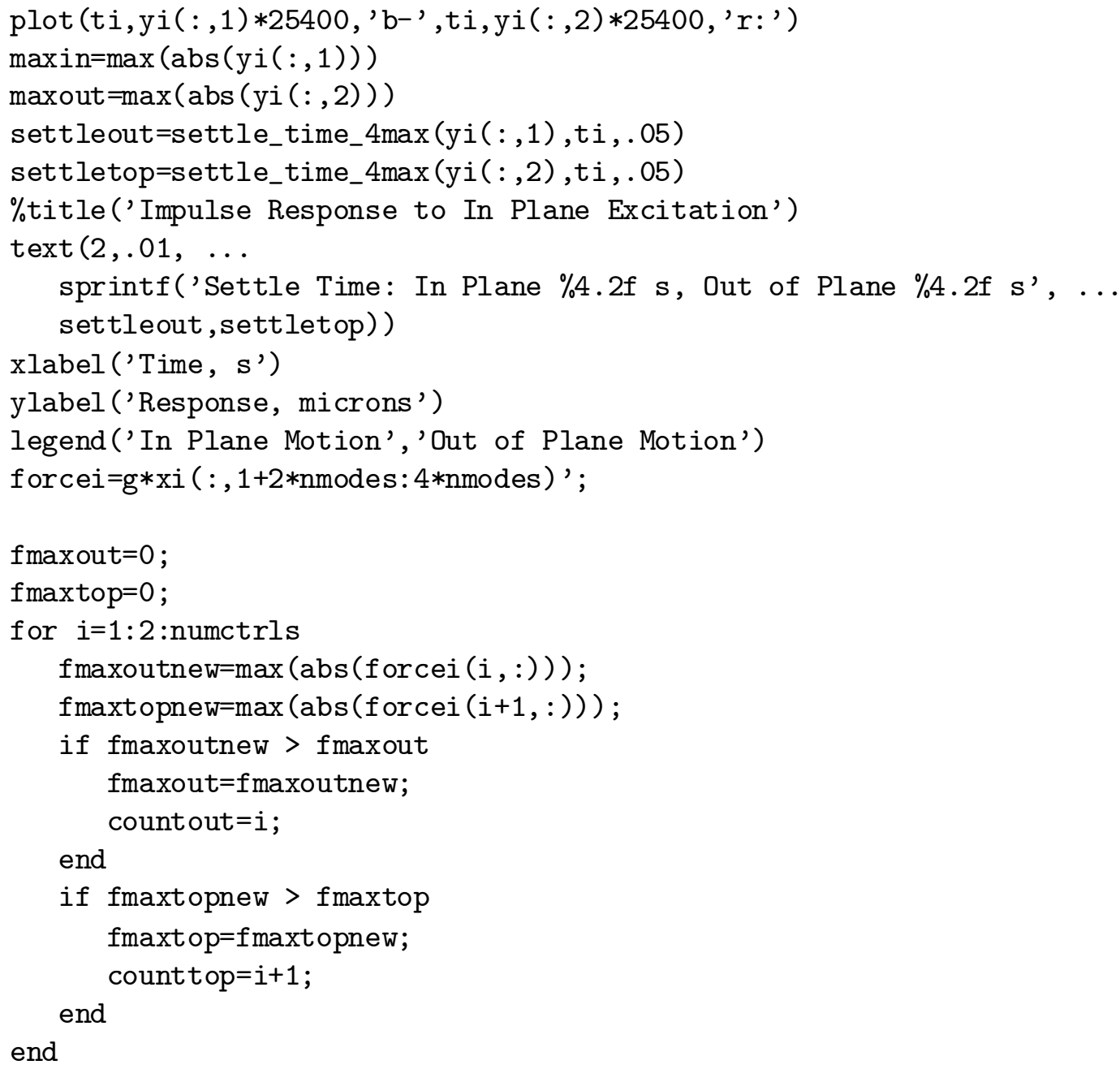




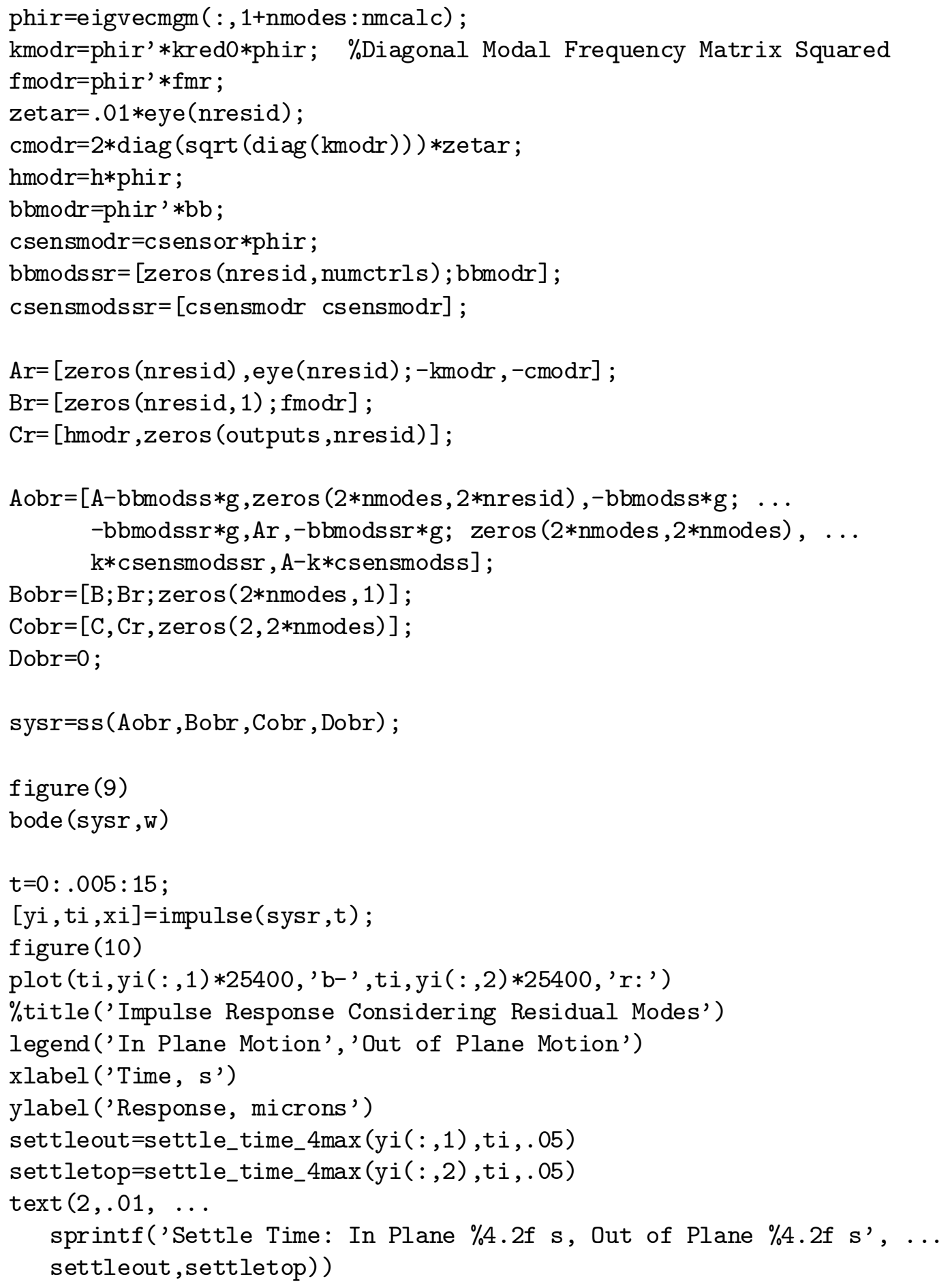




\section{Appendix G}

\section{MATLAB Velocity Feedback Simulation}

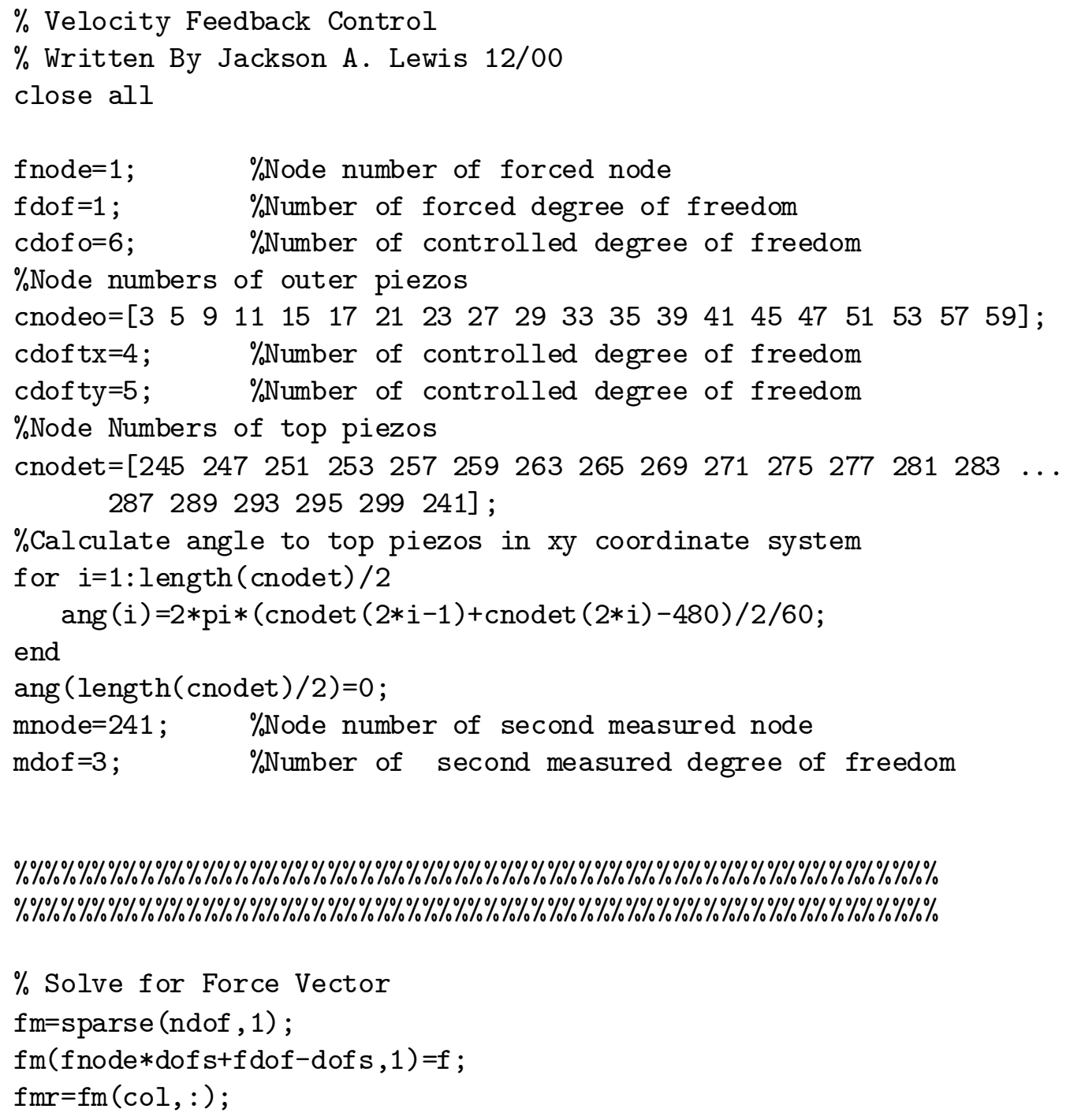




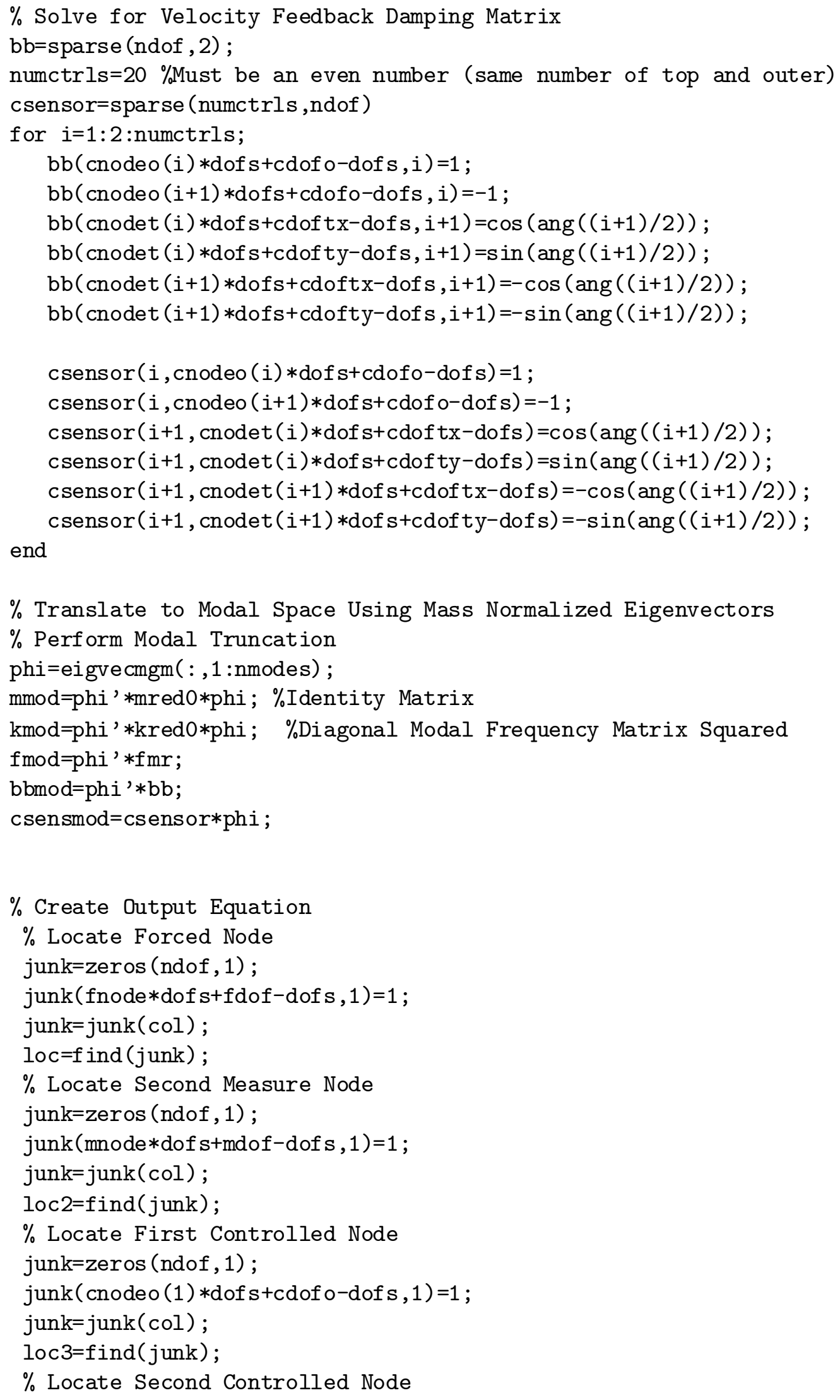




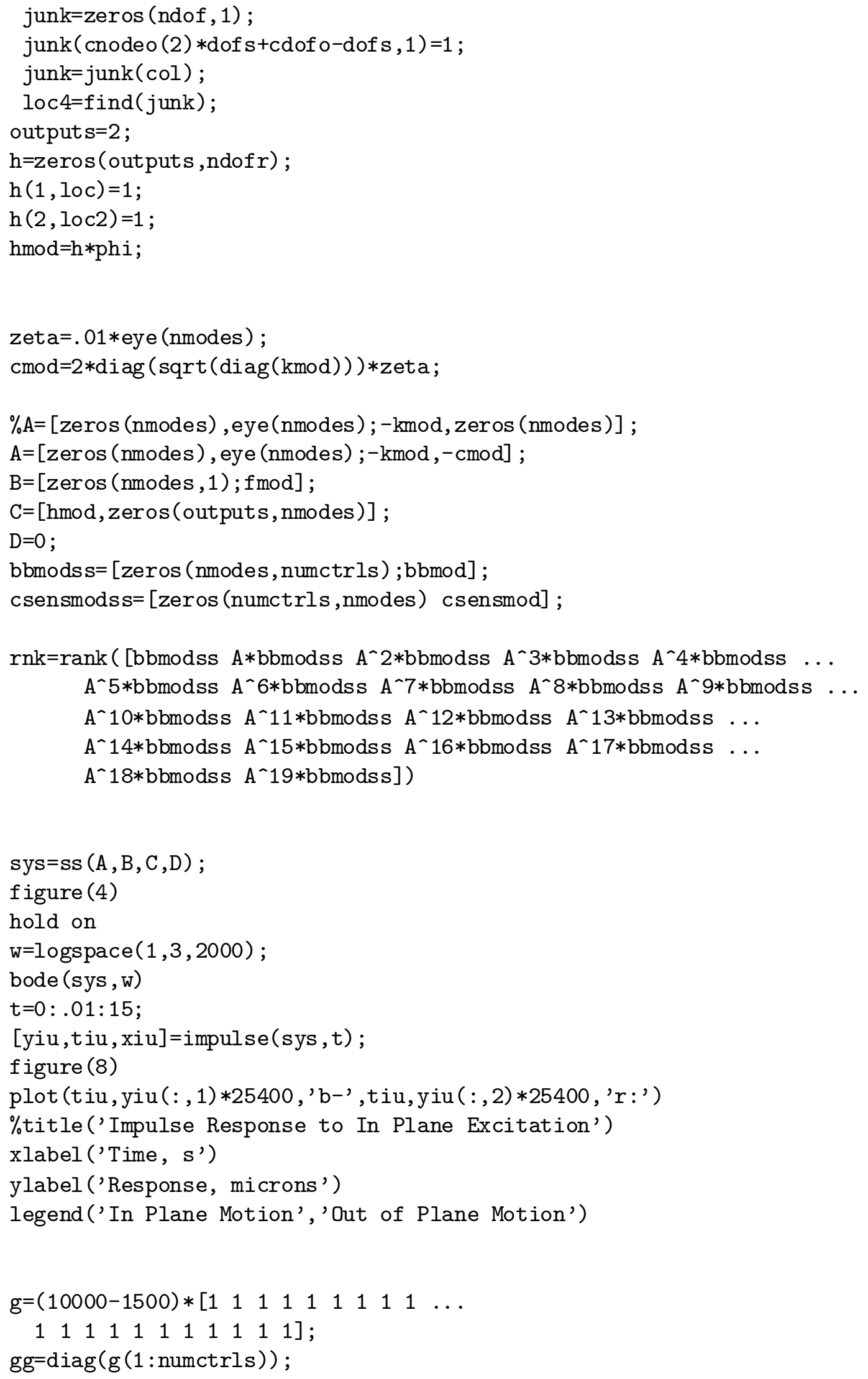




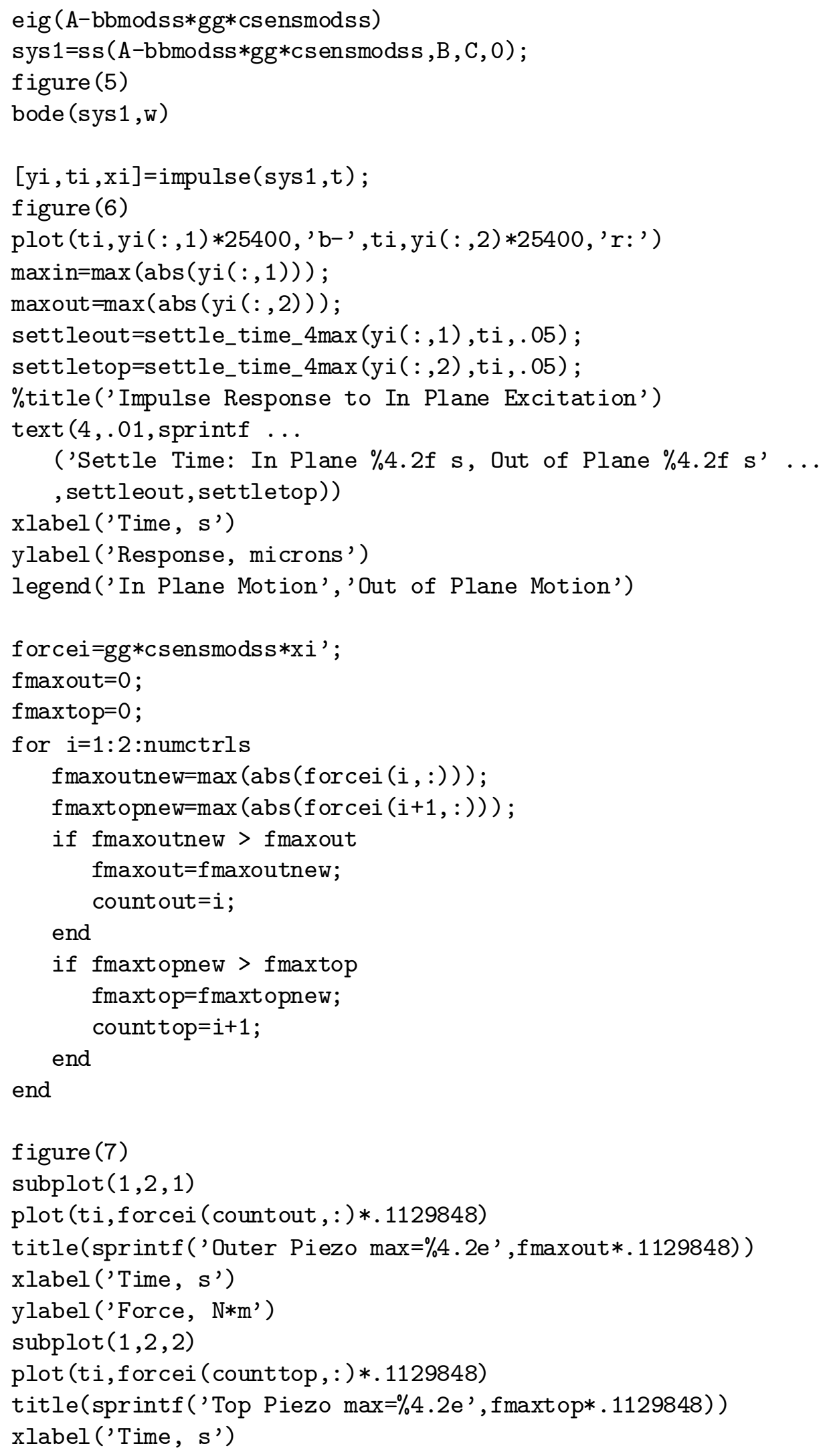


text (3,2e-4, 'Max Possible=3.3e-4 N*m')

ylabel ('Force, N*m') 


\section{Vita}

The author, Jackson A. Lewis, son of Jackson E. Lewis and Jennifer C. Addington, was born on October 27, 1977, in Fairfax, Virginia. He obtained his high school diploma from Falls Church High School, in Falls Church, Virginia, in 1995. He then attended Virginia Polytechnic Institute and State University, in Blacksburg, Virginia, where he earned a Bachelor of Science in Mechanical Engineering, Summa cum Laude, in Honors, in May 2000. Continuing at Virginia Tech under the direction of Dr. Daniel J. Inman, he pursued a Master of Science degree concentrating on structural vibrations. Upon completion of his MS degree in December 2000 he will work for Ball Aerospace in Boulder, CO.

Permanent Address: 9324 Old Burke Lake Rd.

Burke, Virginia 22015

This thesis was typeset with $\operatorname{ATEX} 2 \varepsilon^{1}$ by the author.

\footnotetext{
${ }^{1} \mathrm{AT}_{\mathrm{E}} \mathrm{X} 2_{\varepsilon}$ is an extension of $\mathrm{AT}_{\mathrm{E}} \mathrm{X}$. $\mathrm{H}_{\mathrm{E}} \mathrm{T} \mathrm{X}$ is a collection of macros for $\mathrm{T}_{\mathrm{E}} \mathrm{X}$. $\mathrm{T}_{\mathrm{E}} \mathrm{X}$ is a trademark of the American Mathematical Society. The macros used in formatting this thesis were written by Greg Walker, Department of Mechanical Engineering, Virginia Tech.
} 